\title{
Synthesis of the Acyclic Carbon Skeleton of Filipin III
}

\section{Supporting Information}

Elodie BRUN, Véronique BELLOSTA and Janine COSSY*

Laboratoire de Chimie Organique, Institute of Chemistry, Biology and Innovation (CBI) - UMR 8231 - ESPCI ParisTech/CNRS/PSL Research University, 10 rue Vauquelin, 75231 Paris Cedex 05, France

janine.cossy@espci.fr

Chromatograms for the determination of the ee of $\mathbf{2 4}$

Copies of ${ }^{1} \mathrm{H}$ NMR and ${ }^{13} \mathrm{C}$ NMR spectra 2 


\section{Chromatograms for the determination of the ee of 24}

- $\quad$ Racemic 24

Chiral SFC: column AD-H, $\mathrm{CO}_{2} / \mathrm{MeOH}=99: 1,100$ bars, $4 \mathrm{~mL} / \mathrm{min}$ ).

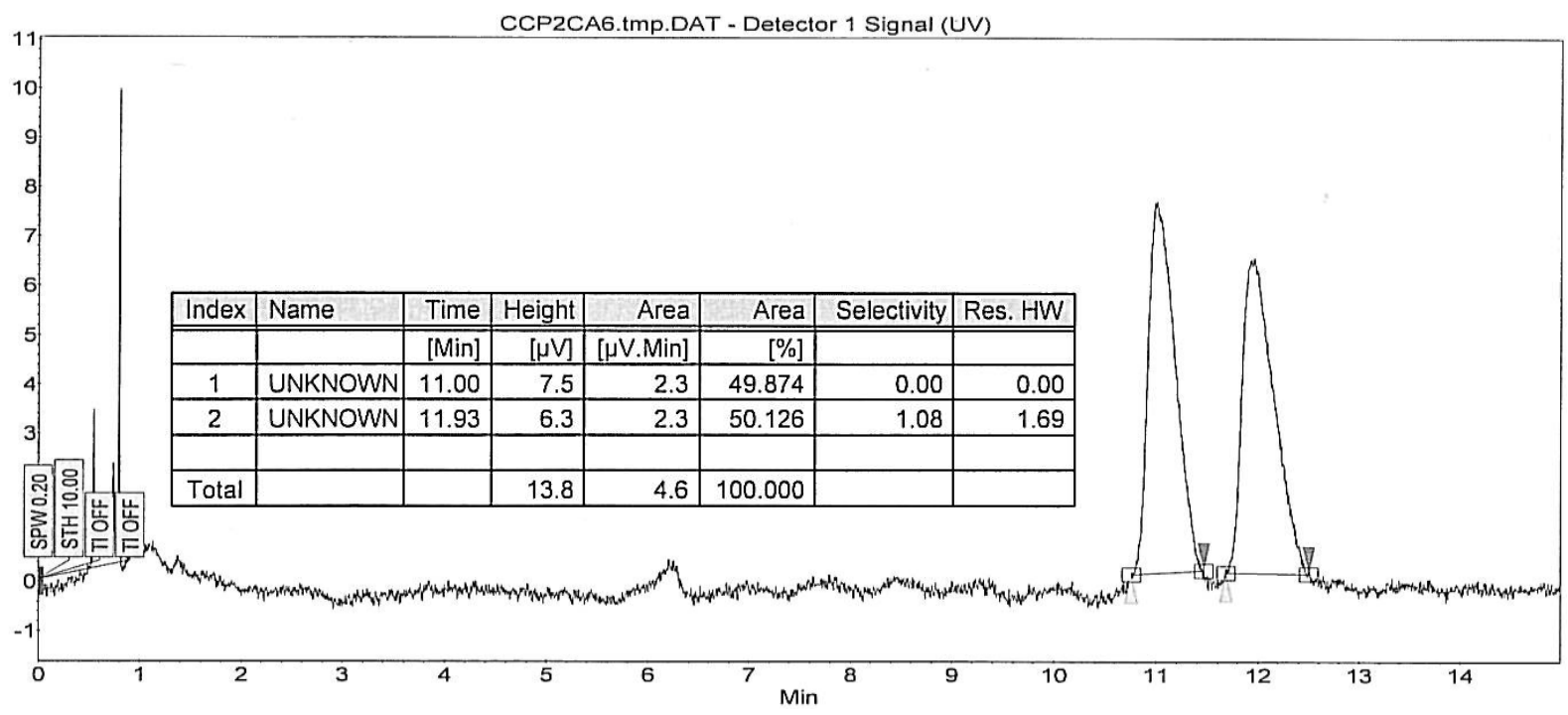

- Enantio-enriched $\mathbf{2 4}$

Chiral SFC: column AD-H, $\mathrm{CO}_{2} / \mathrm{MeOH}=99: 1,100$ bars, $4 \mathrm{~mL} / \mathrm{min}$ ).

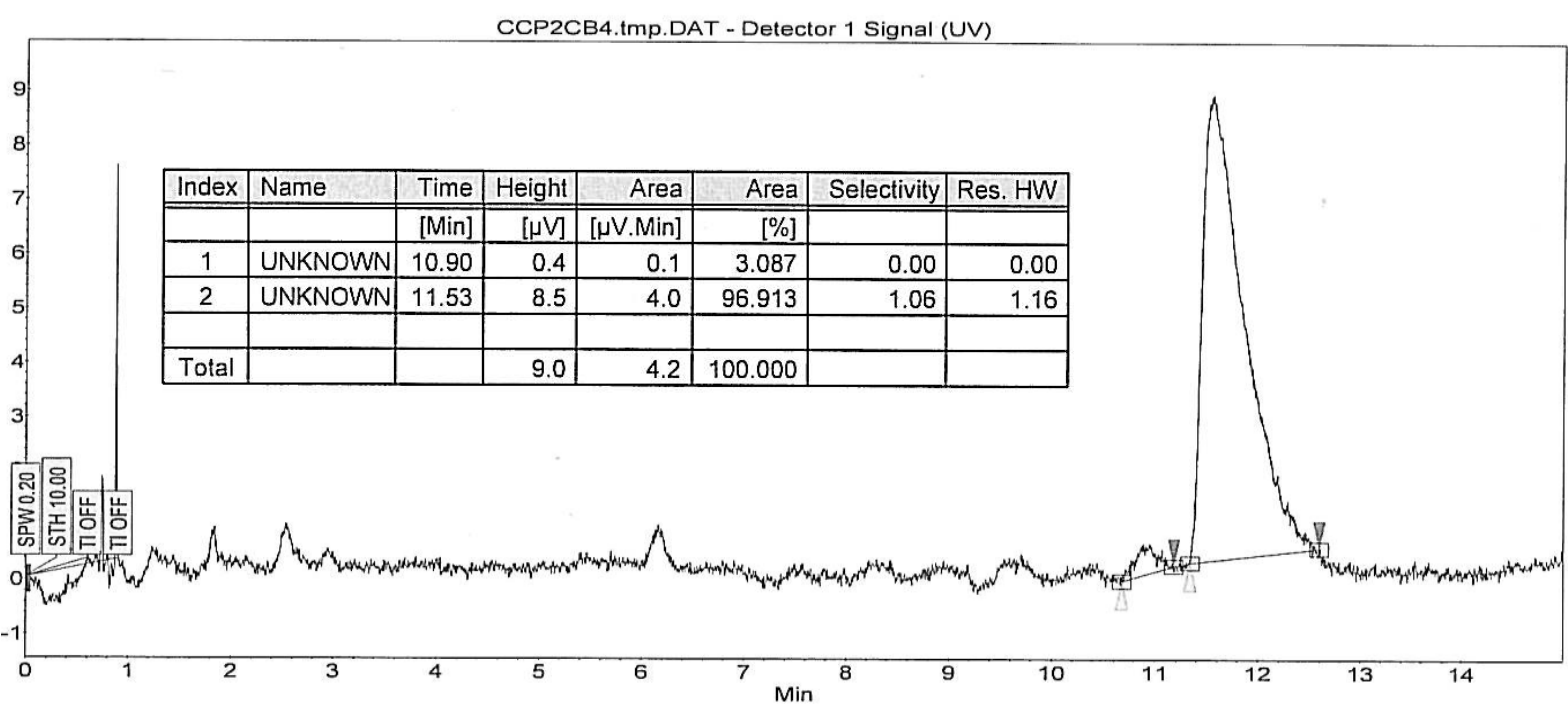

\section{$\underline{\text { Copies of }{ }^{1} \mathrm{H} \text { NMR and }{ }^{13} \mathrm{C} \text { NMR Spectra }}$}




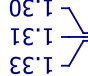

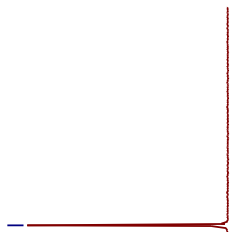

$\mid \begin{gathered}0 \\ 0 \\ 0 \\ 0 \\ 0 \\ 0 \\ 0 \\ 0 \\ 0 \\ 0 \\ 0\end{gathered}$

$\varepsilon \varepsilon^{\prime}[-$

${ }^{96} \cdot \mathrm{I} \backslash$

96. I

6I't

II'

SZ'

$t \mathrm{t}^{\circ} \mathrm{s}$

$t t^{\circ} \mathrm{s}$

$96^{\circ} s$

$96 \mathrm{~s}$

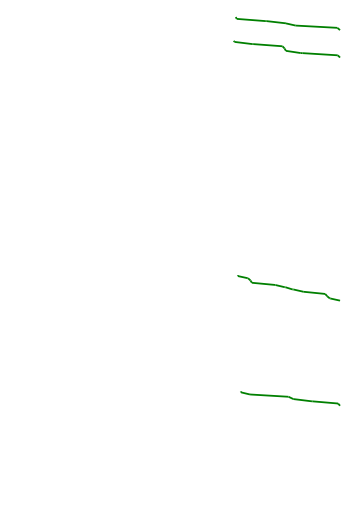

६क्ष

$+9^{\circ} 9$
$+9^{\circ} 9$

$\angle 9^{\circ} 9$

$89^{\prime} 9$

69.9

L $\angle$ ' 9

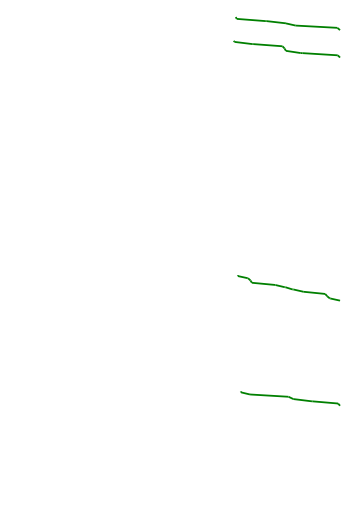

SI $L$

SI' $\angle$ -

$\left.8 \mathrm{I}^{\circ} \mathrm{L}\right]$

$8 \mathrm{I}^{\circ} \mathrm{L}$

$\angle Z Z^{\circ}$
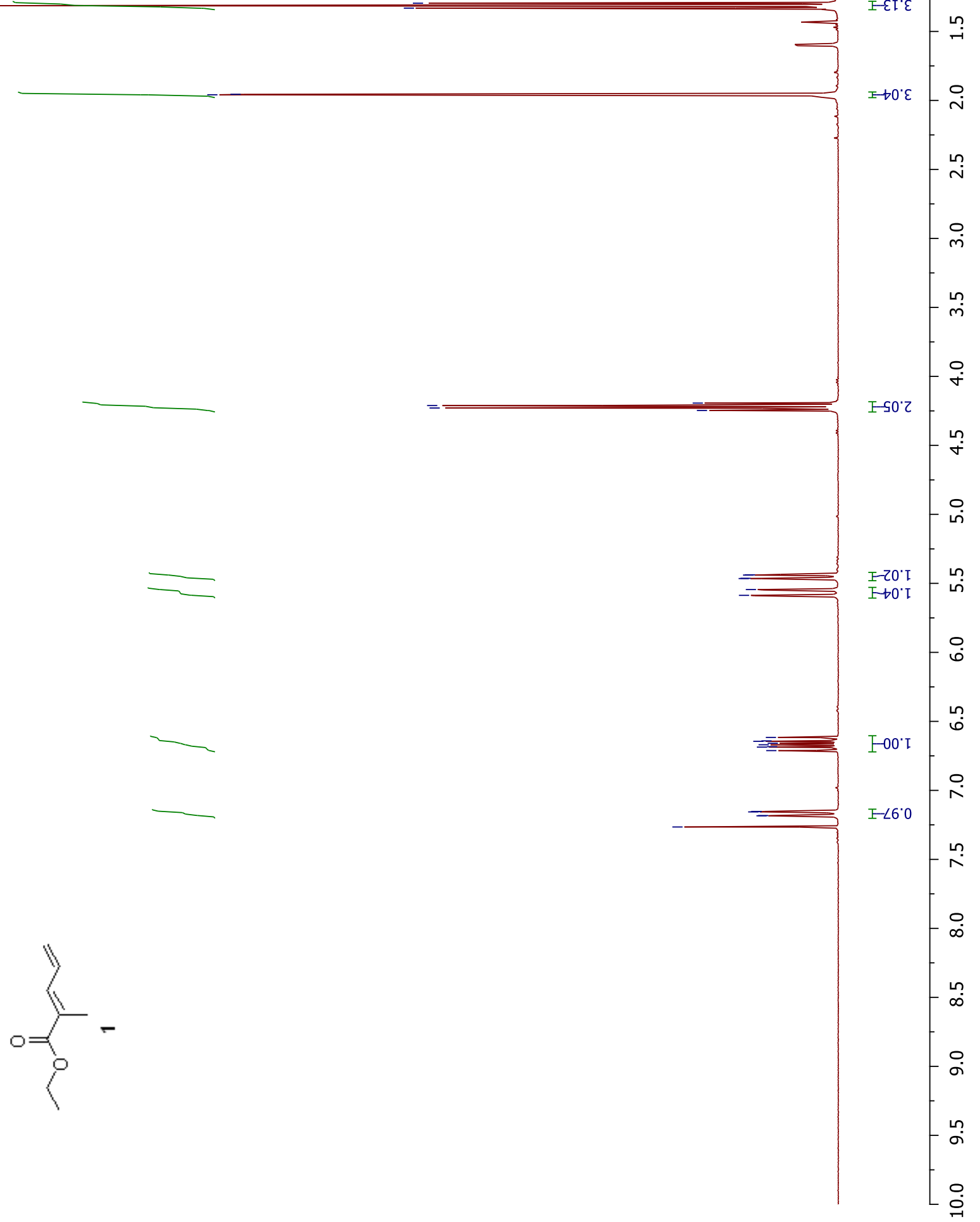


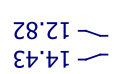

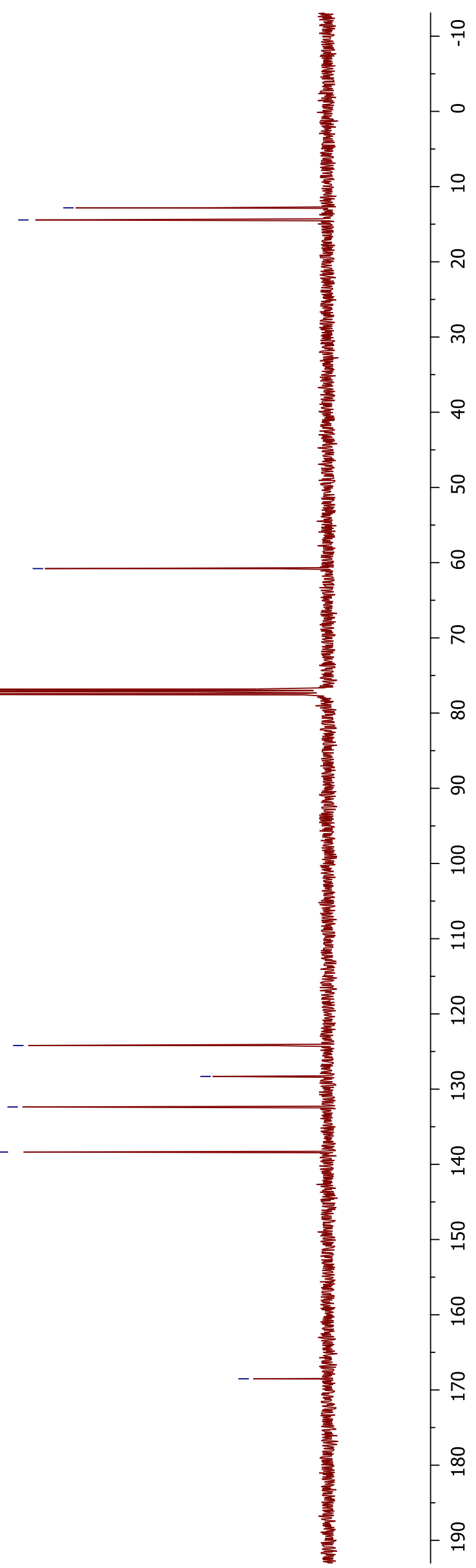

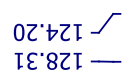

$t 8^{\prime} 9 L$

$9 T^{\circ} \angle L$

$6<\cdot 09-$

8

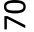

$\infty$

요

움

욱

్ㅗ

IE' 8 ZI -
$\angle \varepsilon^{\prime}$ 'ZI

9ะ $8 \varepsilon I-$

zs.891- 
So'z-

ว०॰ $\varepsilon-$

$\varepsilon 0^{\circ} \circ$
$\angle 0^{\circ} \circ$

IZ'॰

$\forall \angle S T$

हैำ

s9. 9 -

$59^{\circ} 9$

99.9

69.9

$69^{\circ} 9$

ZI' $\angle$

ZI: $\angle-$

SI $\angle-j$

SI $\angle J$

$9 I^{\circ} \angle$

$8{ }^{\circ} \angle-L$

$8 \mathrm{I}^{\circ} \mathrm{L}$

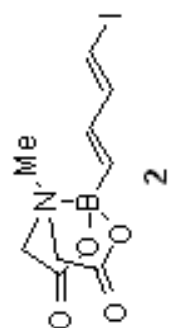

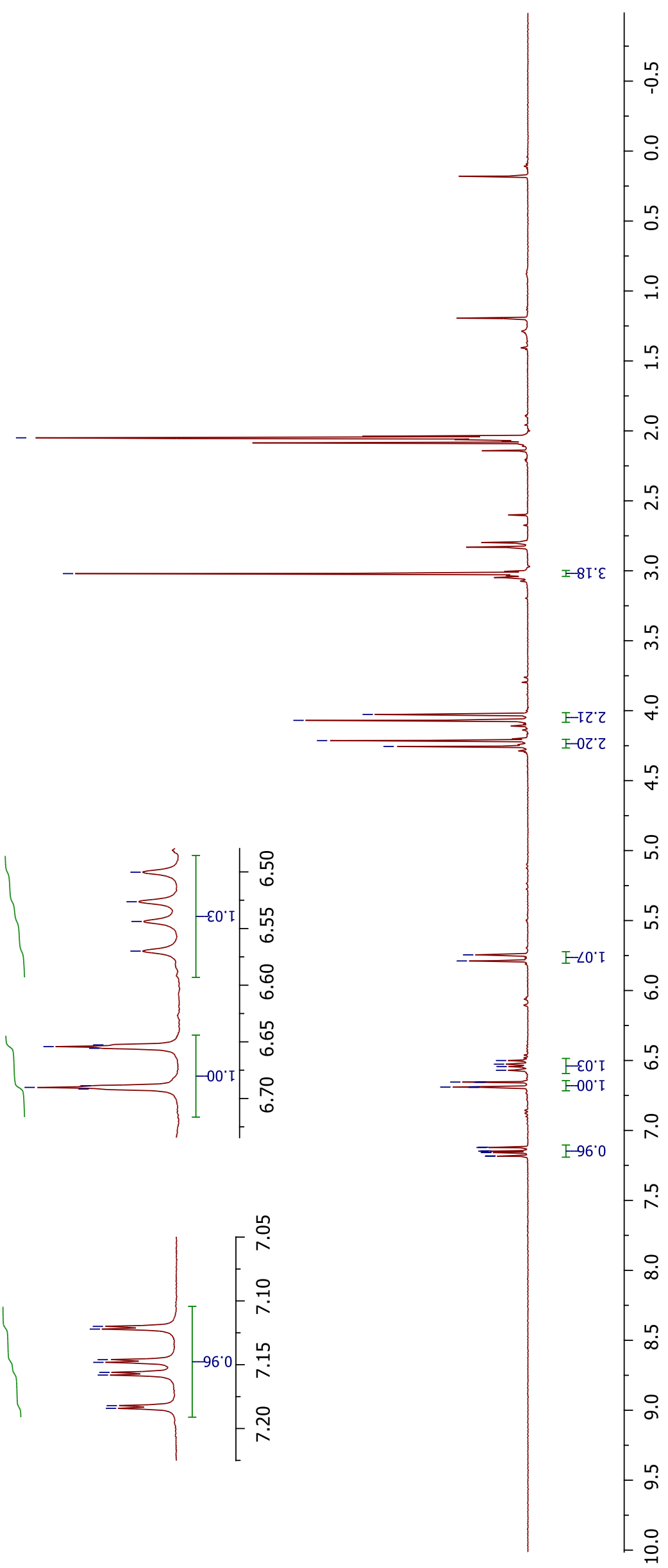




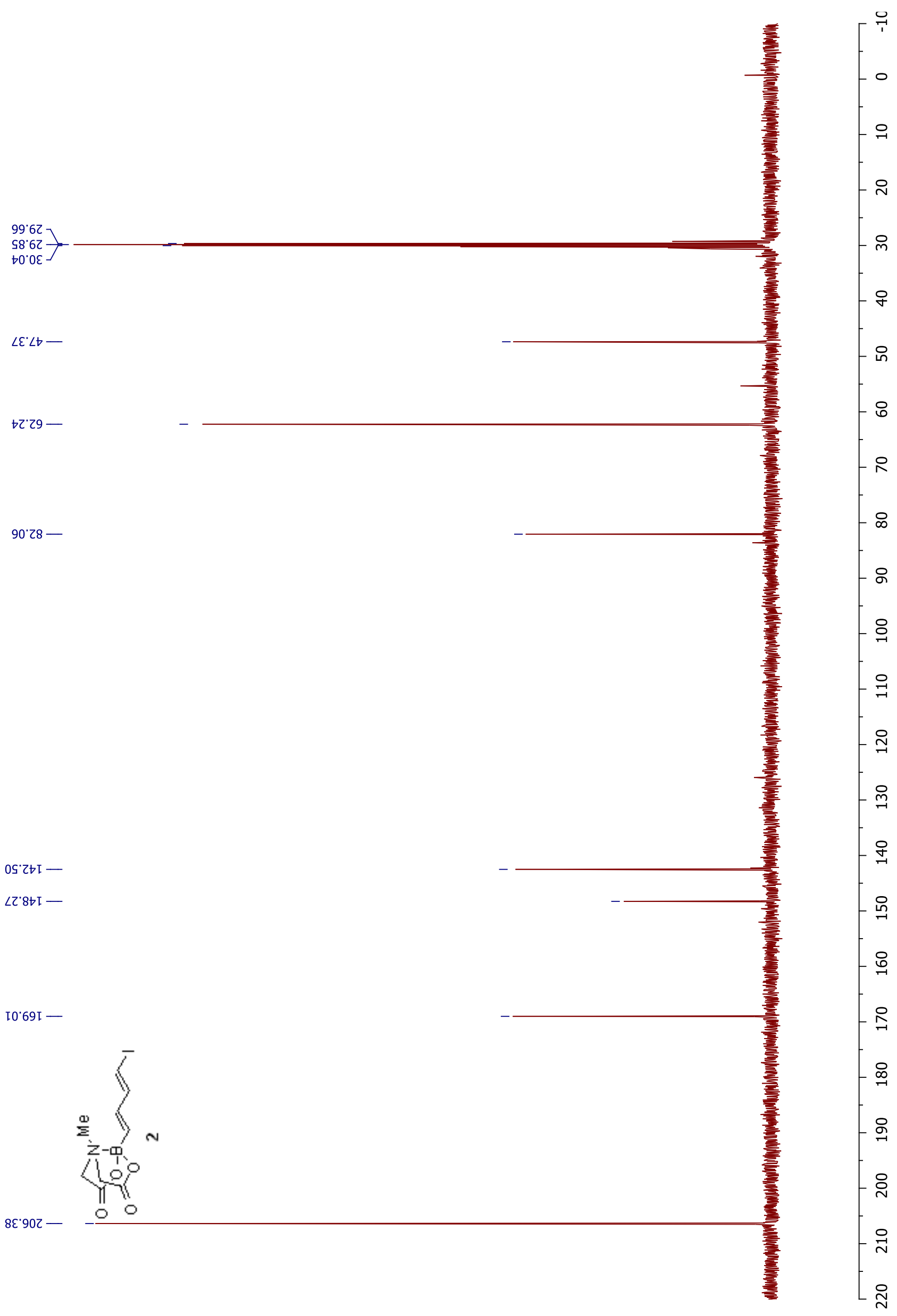




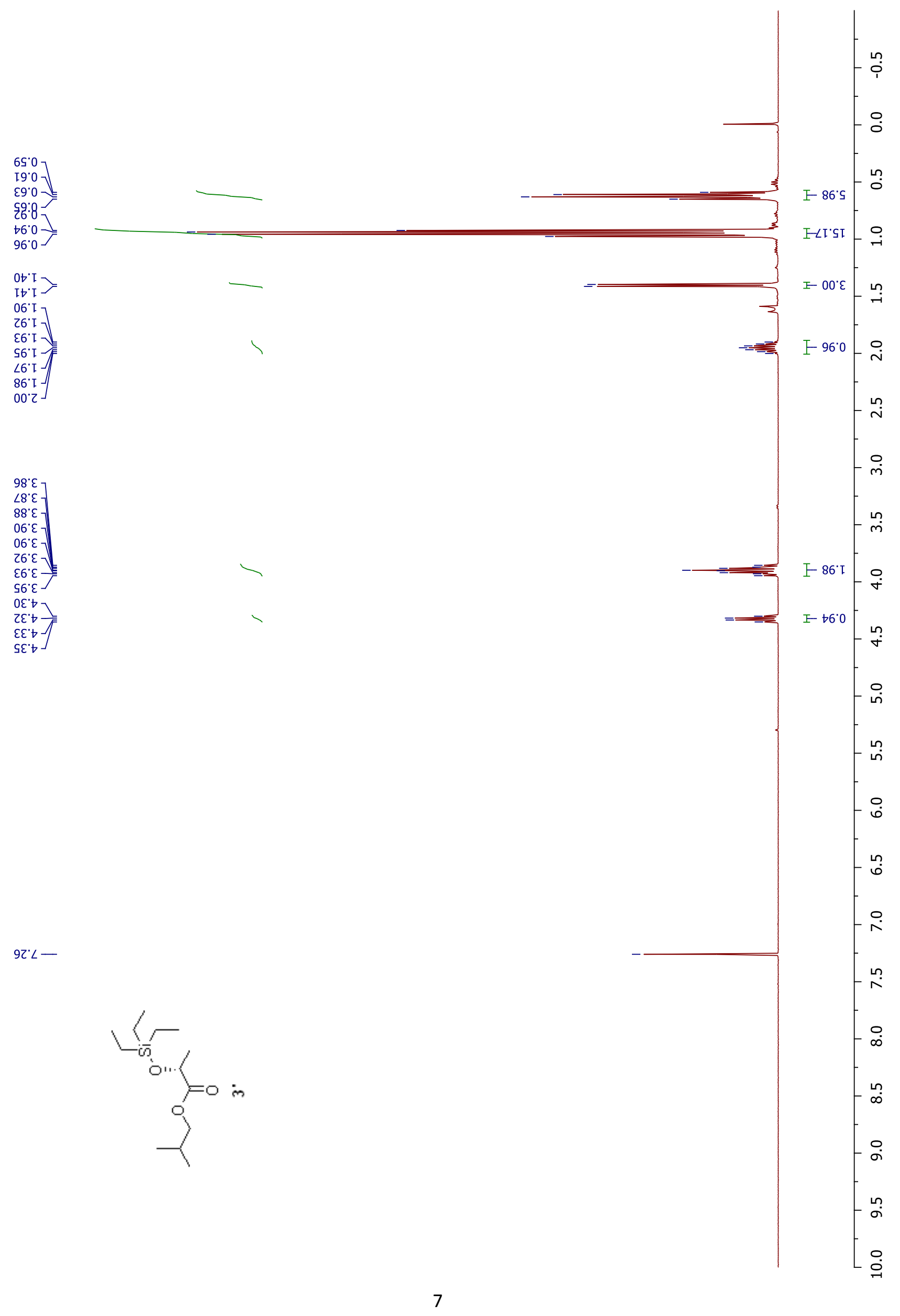



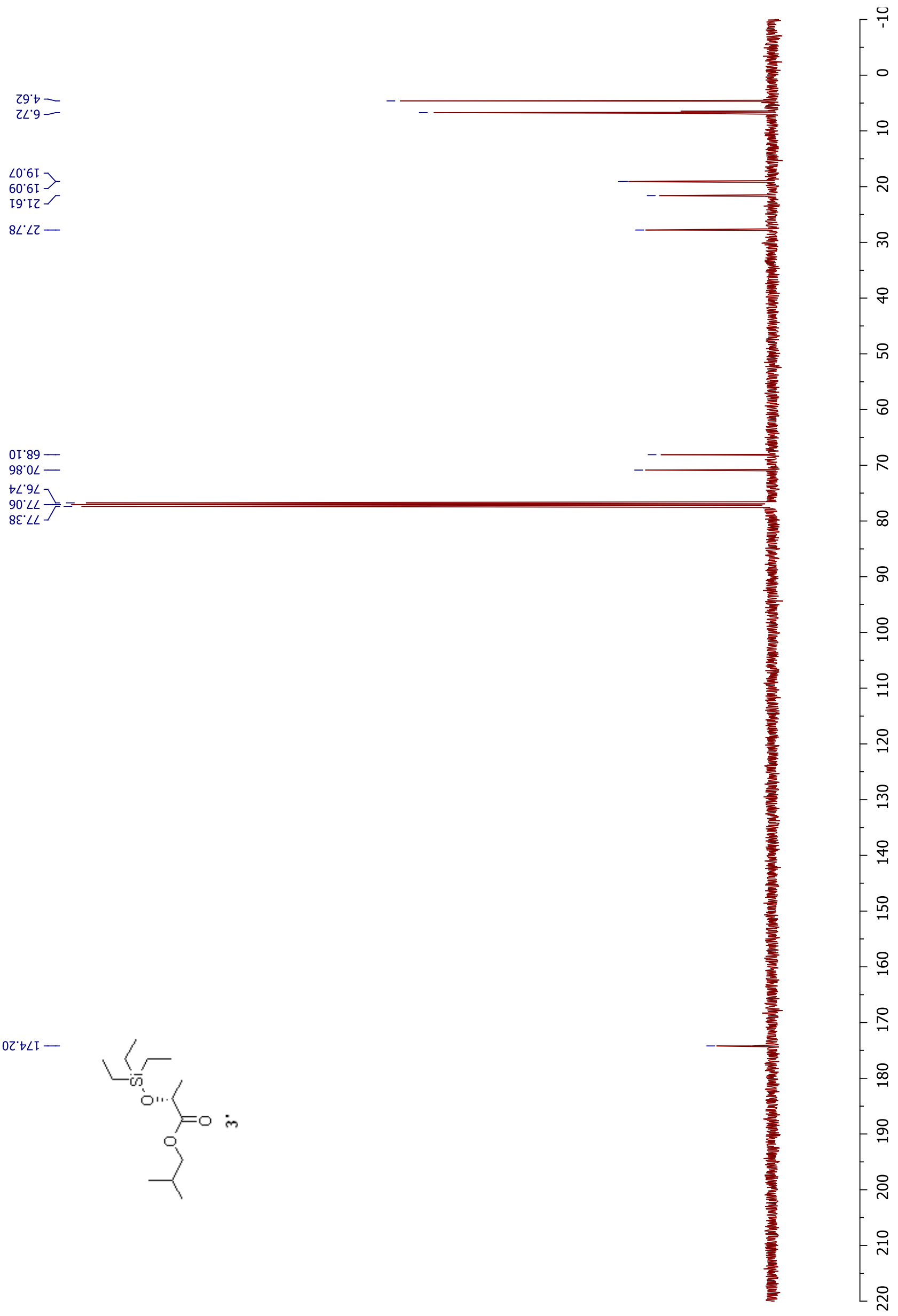


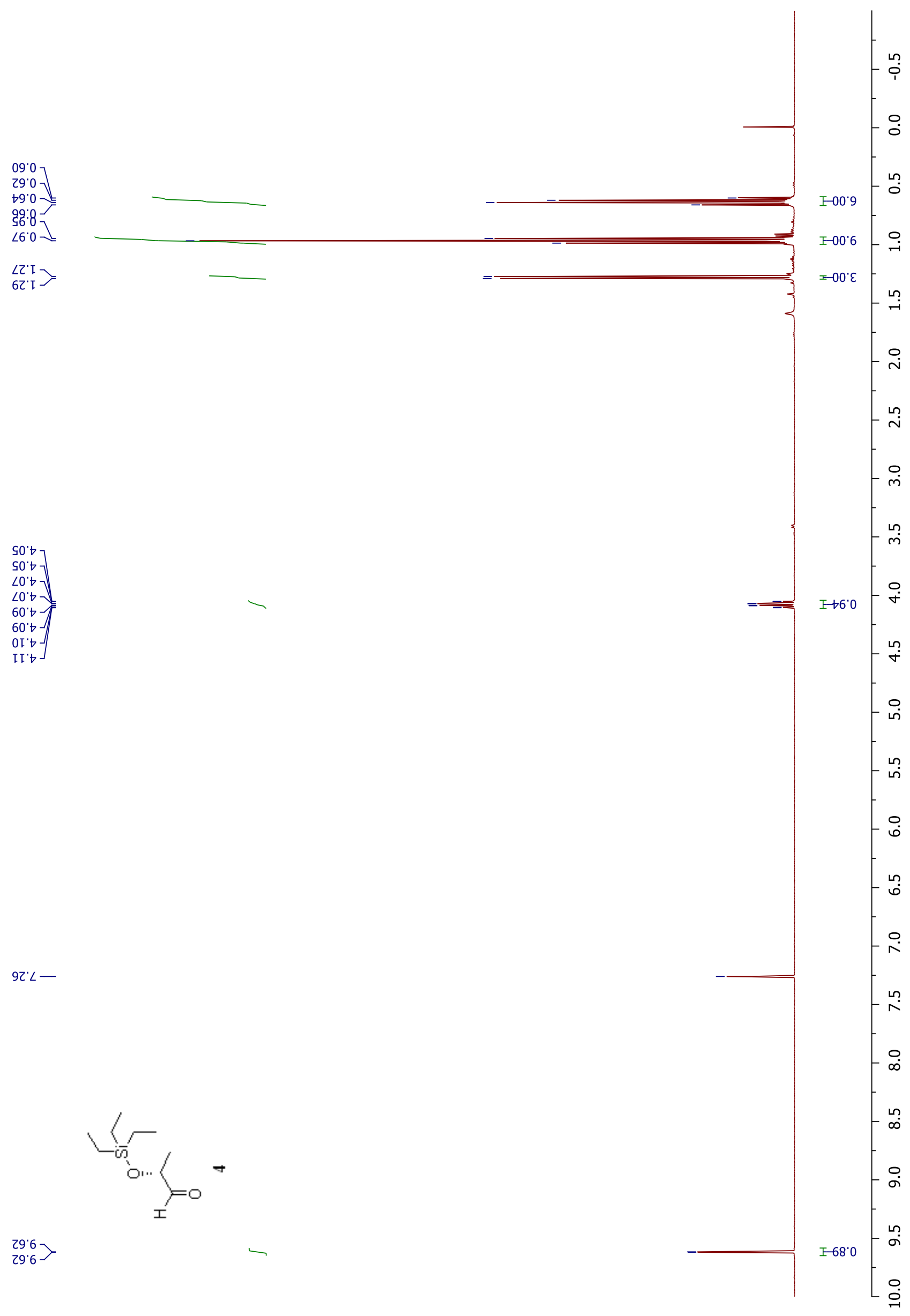




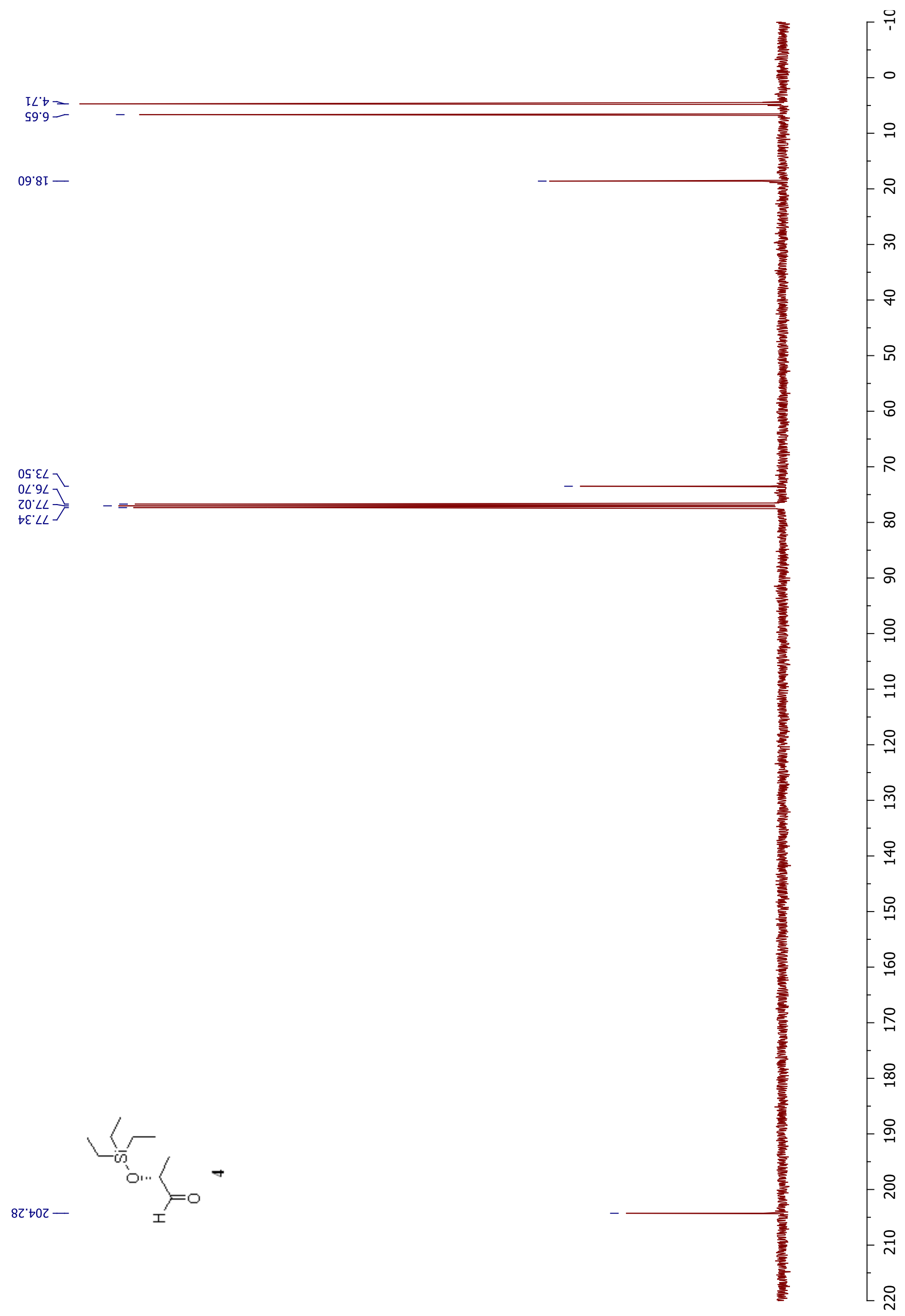




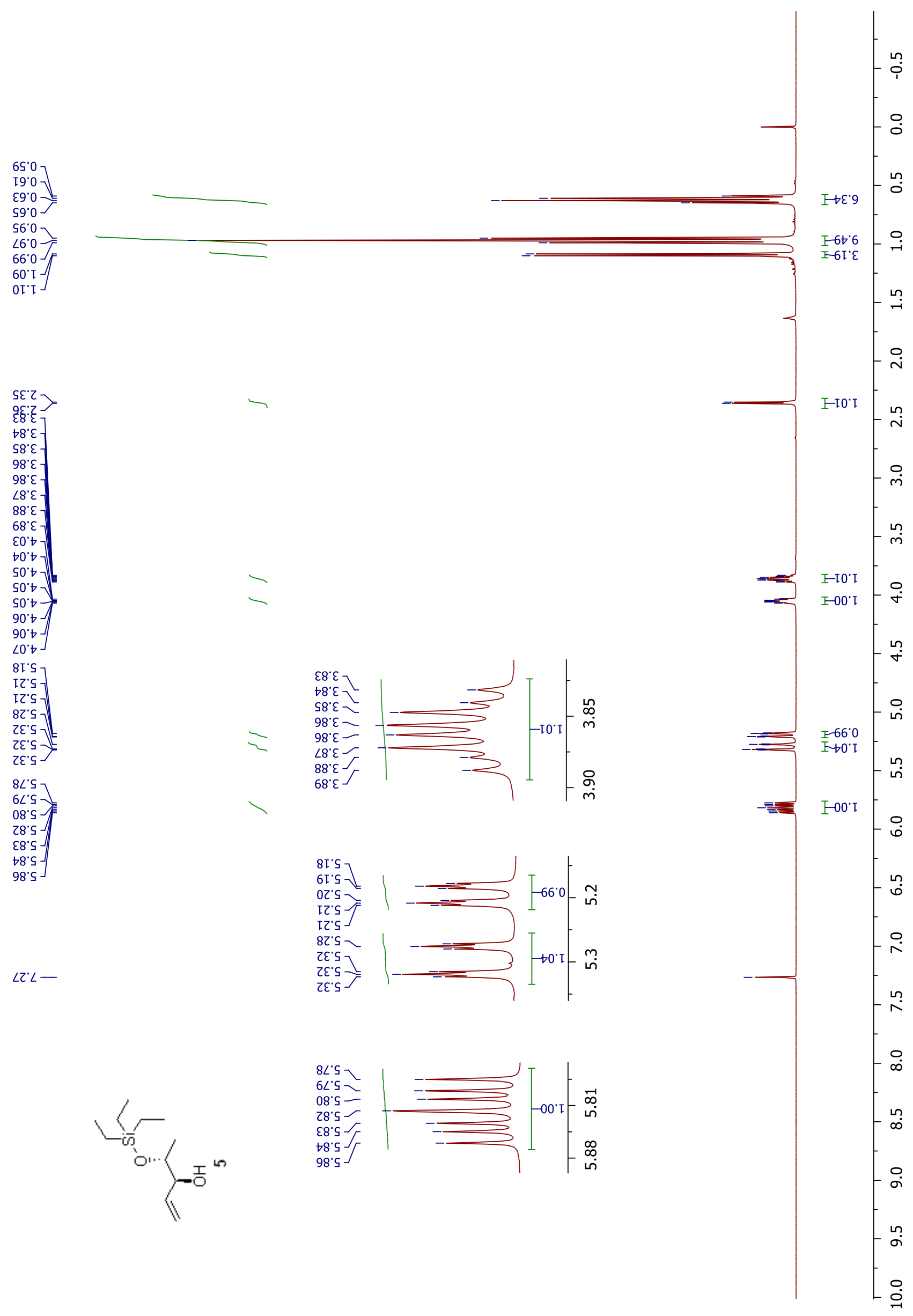


$76 \circ$

$6 t^{\circ} \angle I-$

$60^{\circ}[\angle-$
$0 L^{\circ} 9 \angle$

$0 L^{\prime} 9 L$
$\forall L^{\prime} 9 L$

$90^{\circ} L L-$
$8 \varepsilon^{\circ} \angle L$

$\angle$ เ゚. I -

6S.9عI -

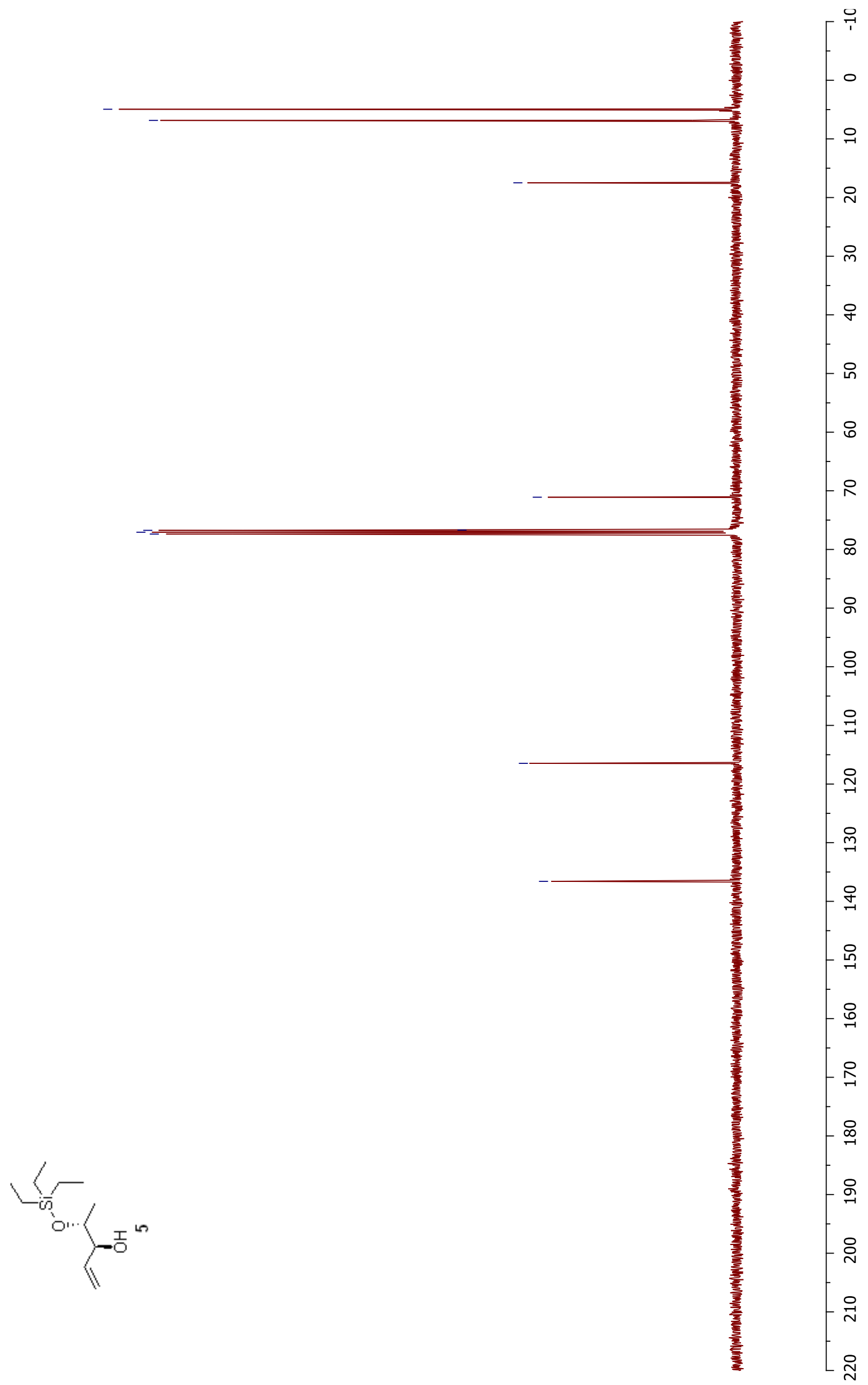




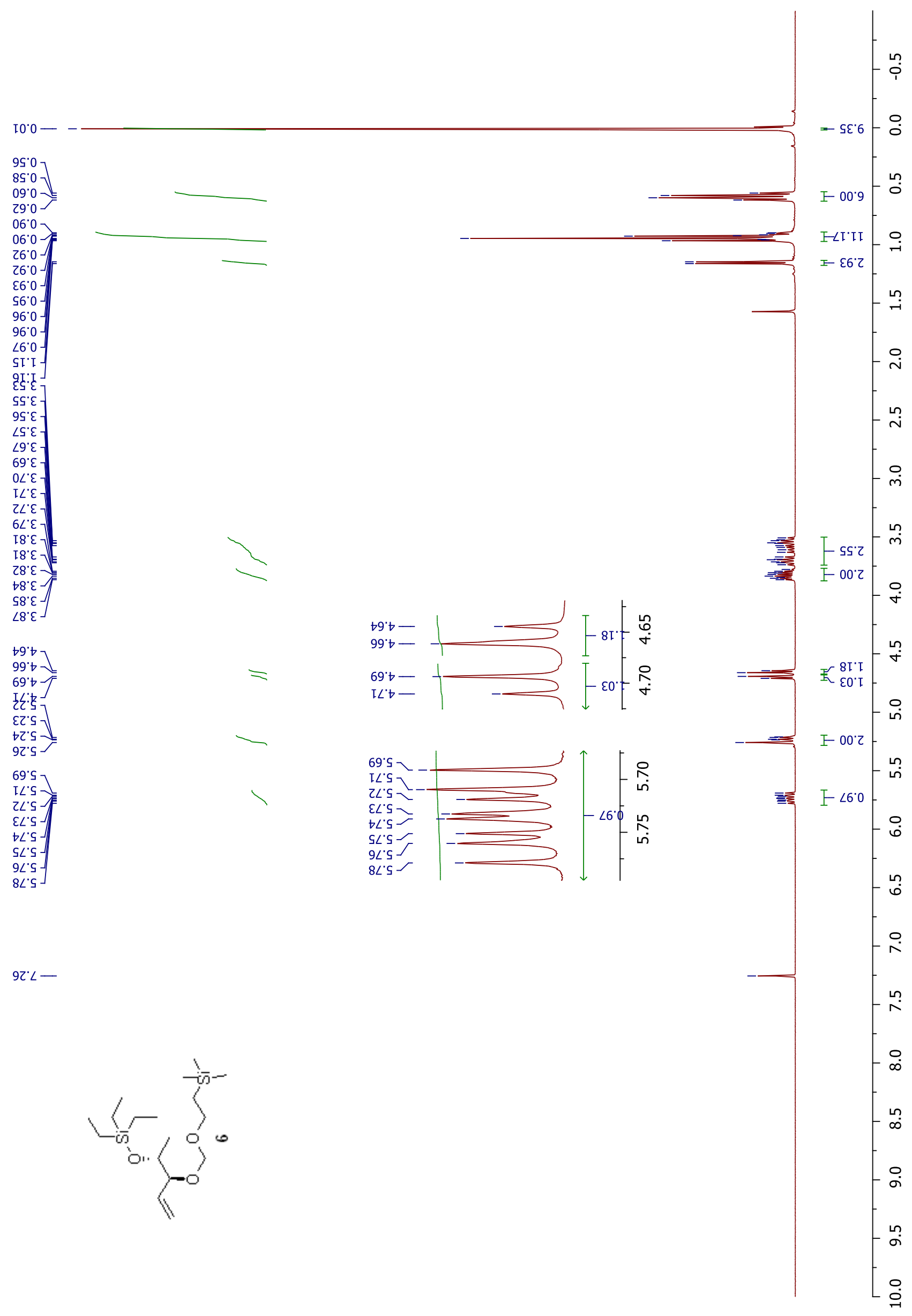



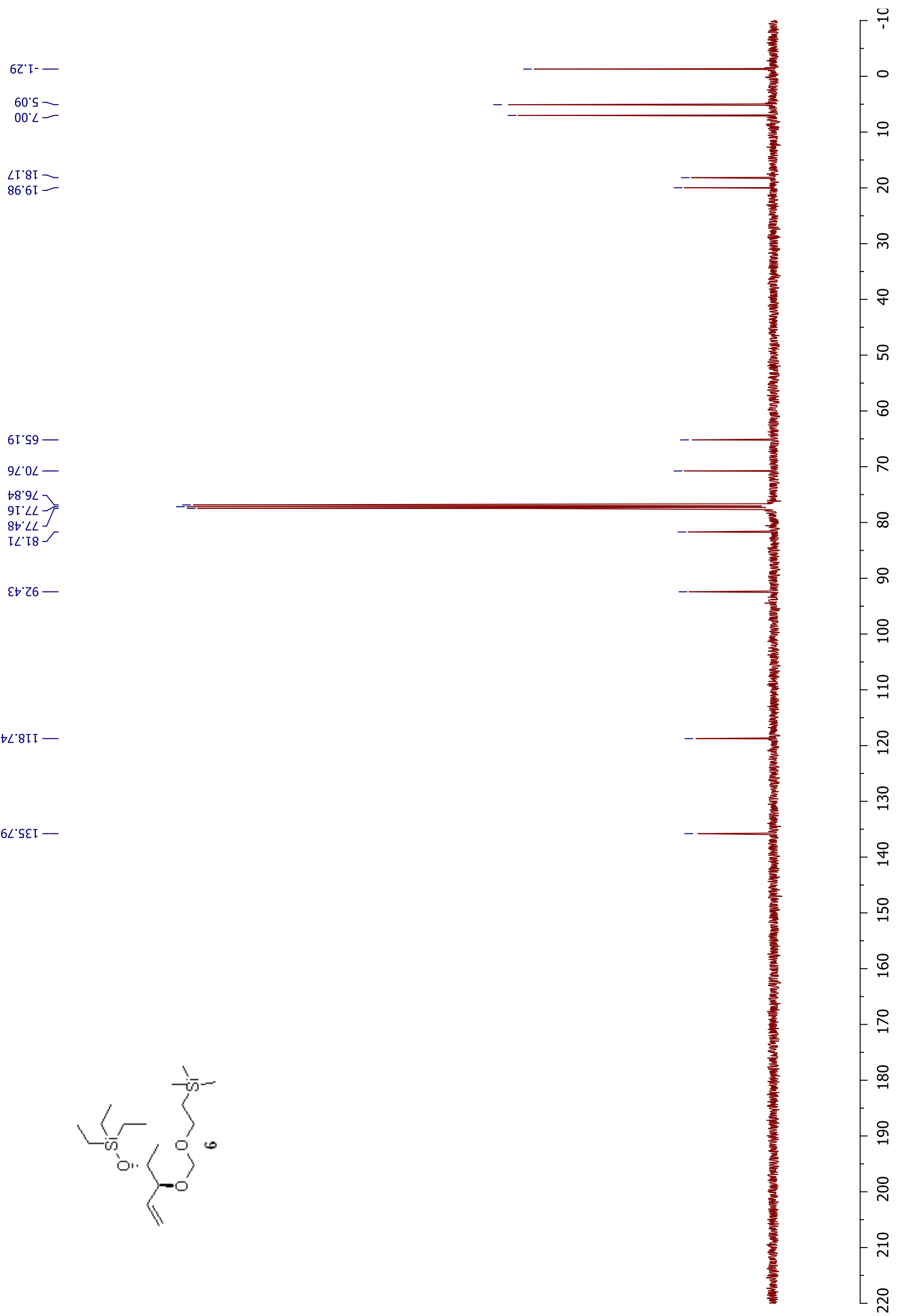

$86.61-$

6I'S9-

$9 \angle{ }^{\circ} O L-$

$\triangleright 8^{\circ} 9 L$

$91^{\circ} \angle L J$
$85^{\circ} \angle L$

IL'T

Et'26-

$t \angle ' 8 I-$ 


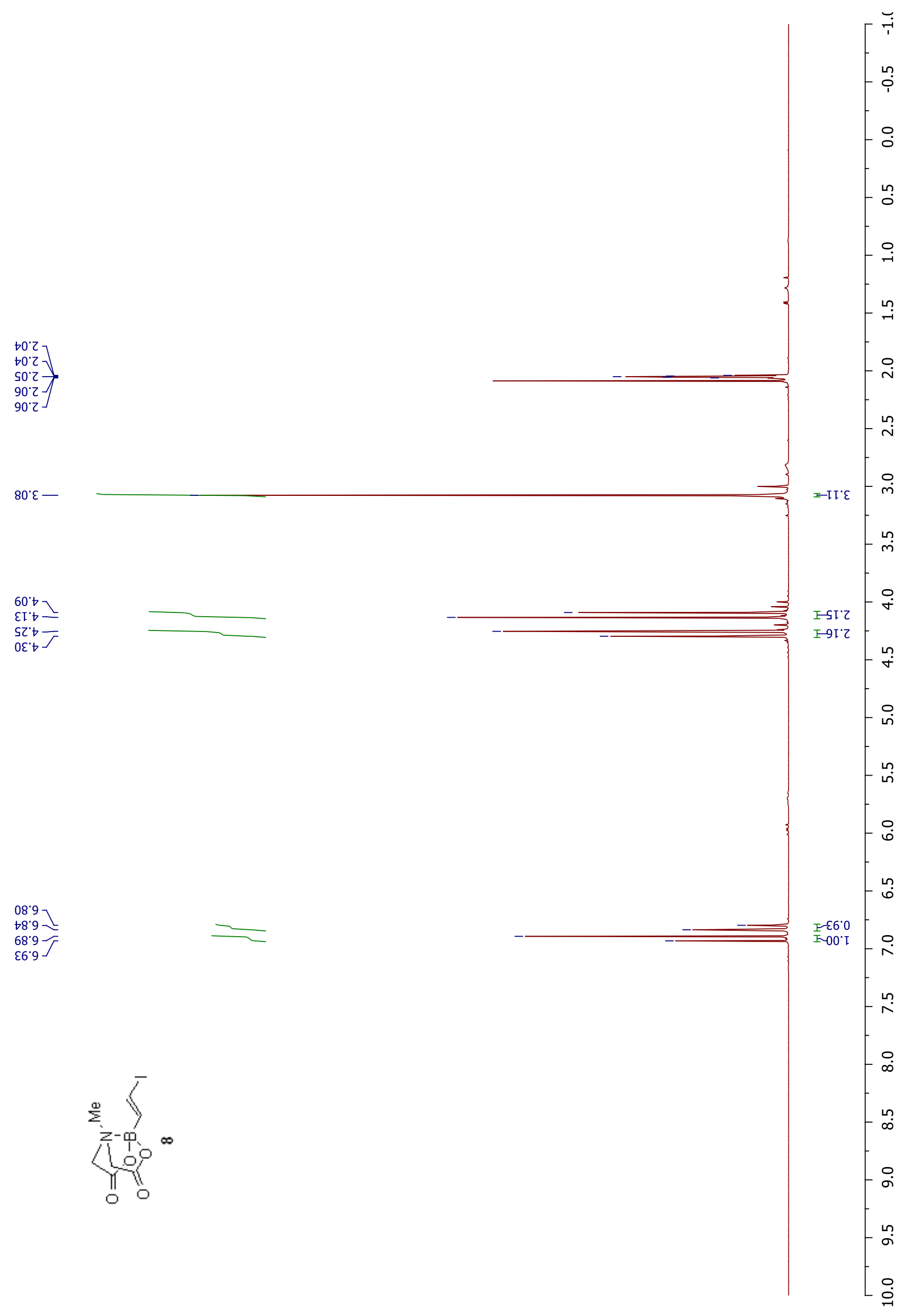




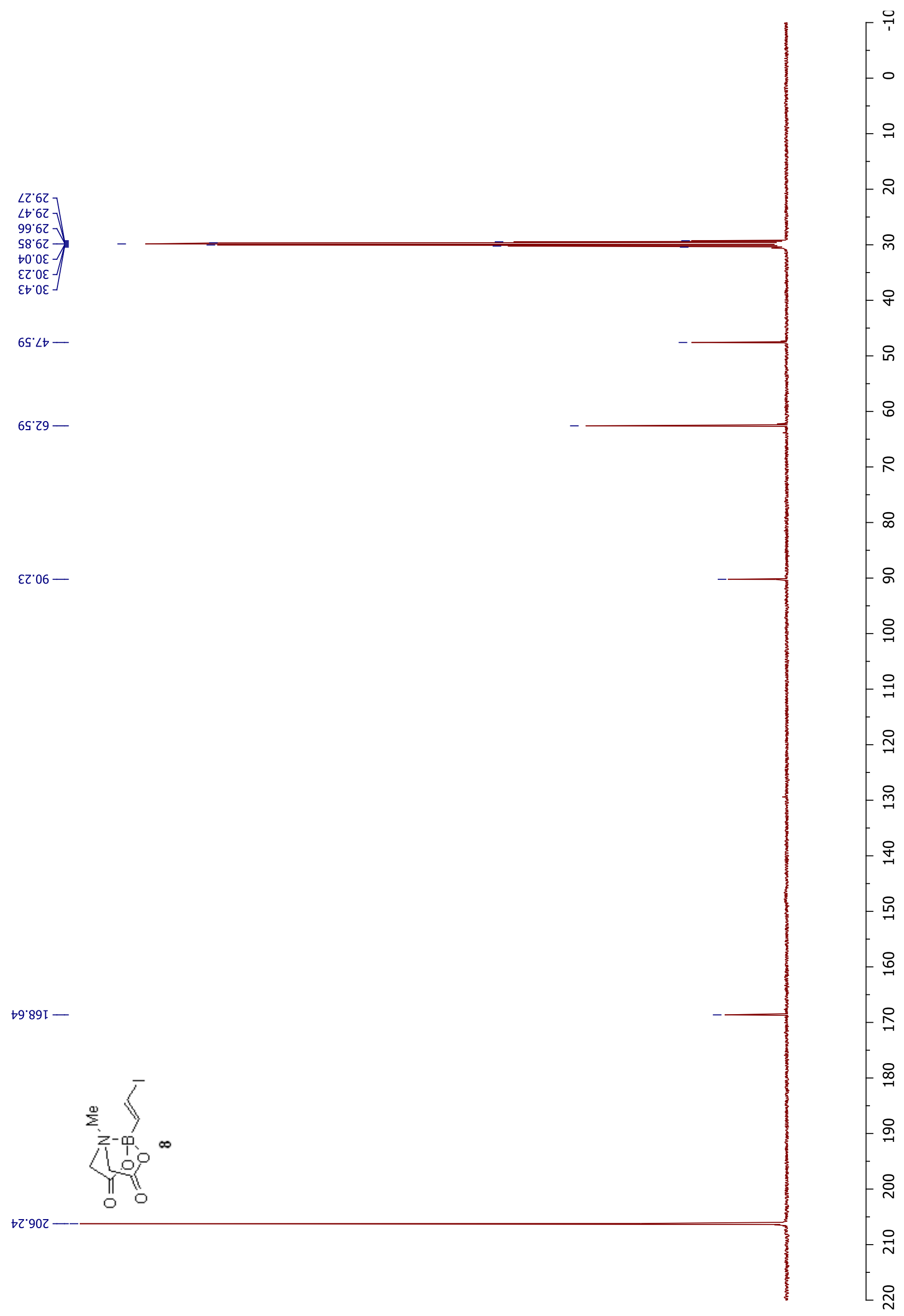




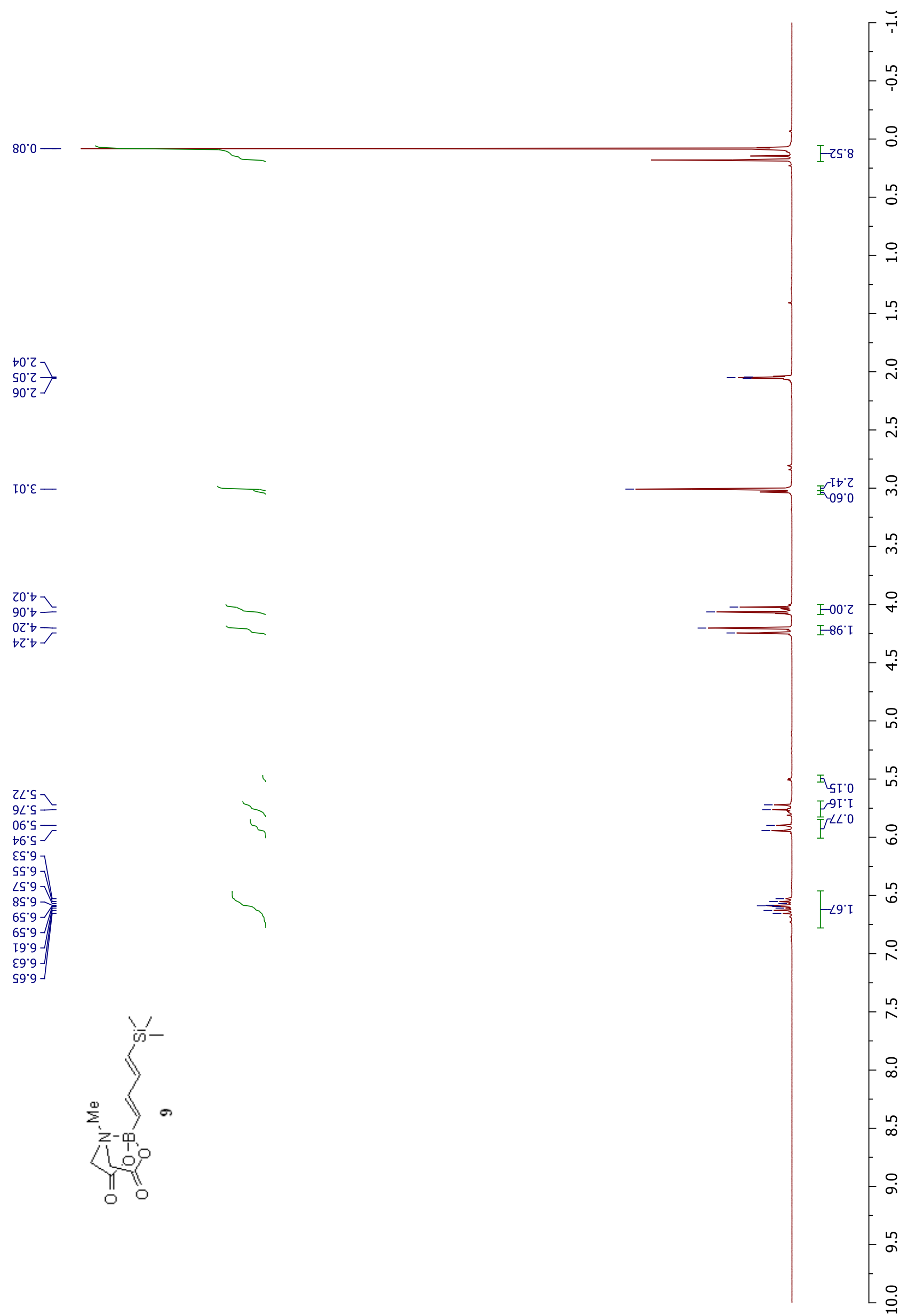


9Z' T- -

$\left.\left.\begin{array}{l}9 t^{\circ} 6 z \\ 90^{\circ} 6 z \\ 58^{\circ} 6 z \\ 0^{\circ} 0 \varepsilon- \\ \varepsilon Z^{\circ} 0 \varepsilon \\ \varepsilon t^{\circ} 0 \varepsilon\end{array}\right]\right\}$

o६'२9-

II'SEI -

${ }^{\circ} \mathrm{S} b \mathrm{I}-$

$\angle Z \cdot \angle T I=$

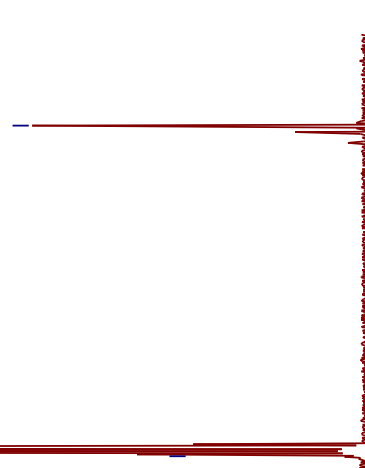

[군
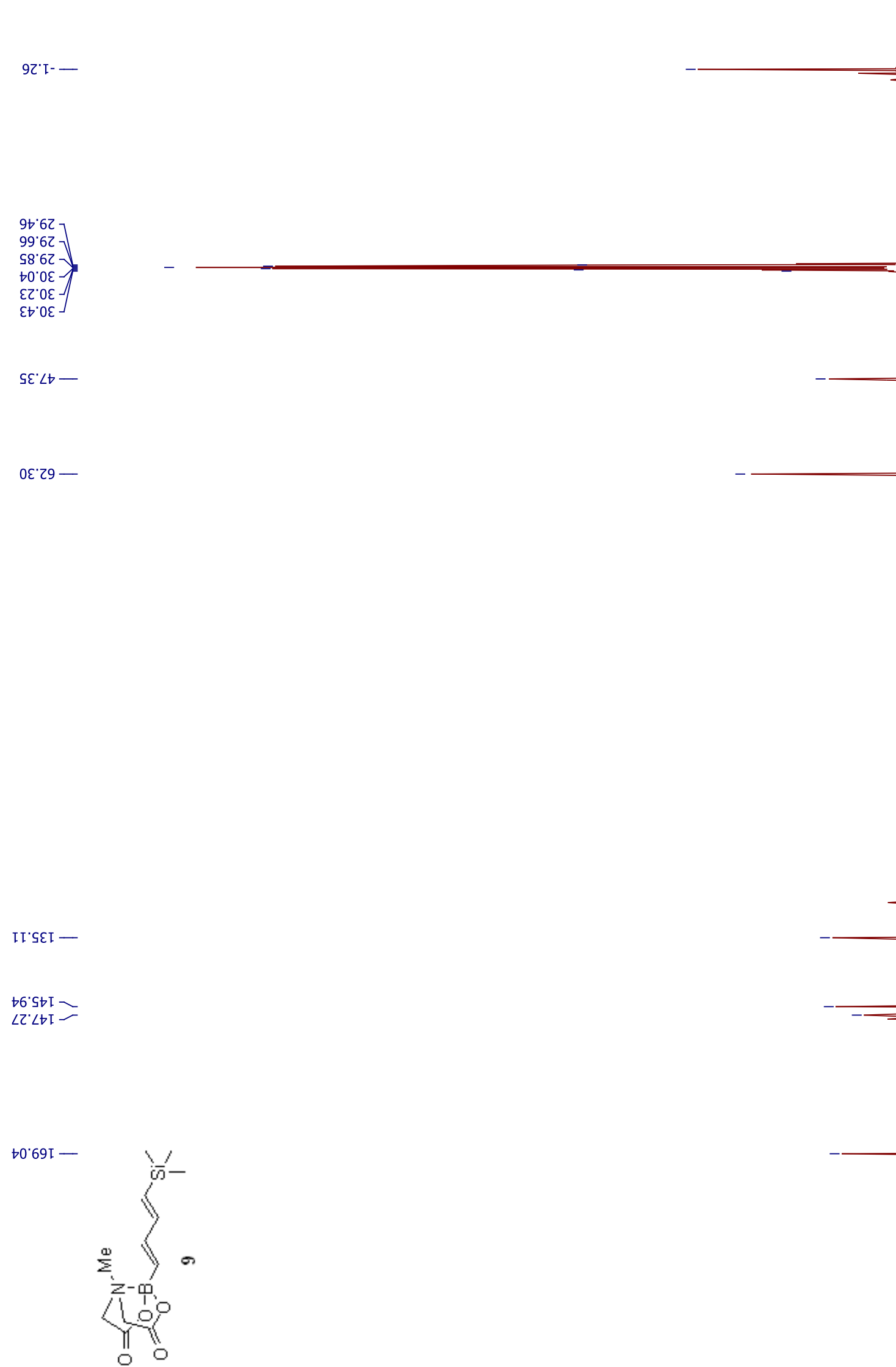

0z'90z

$\circ$ 


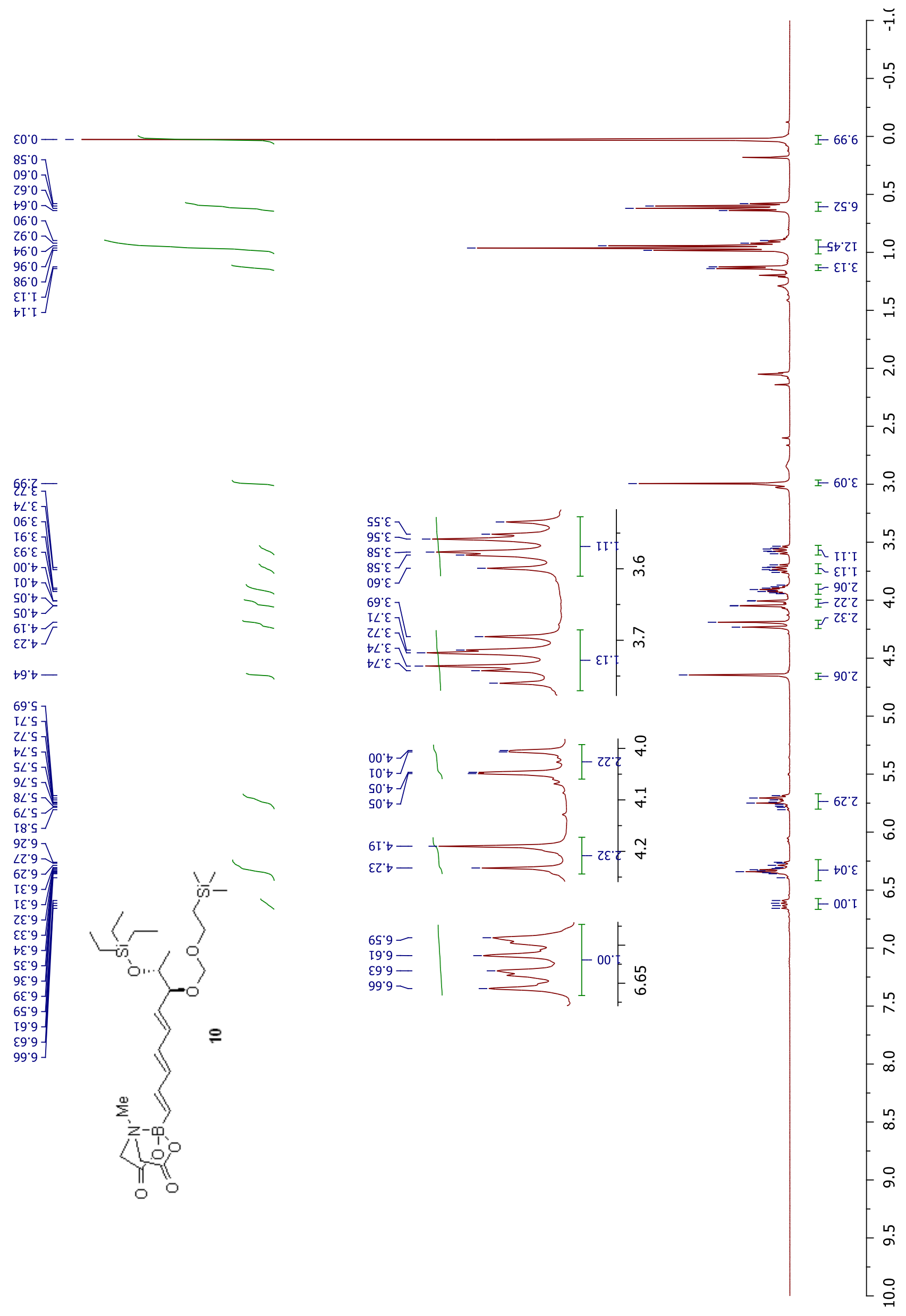




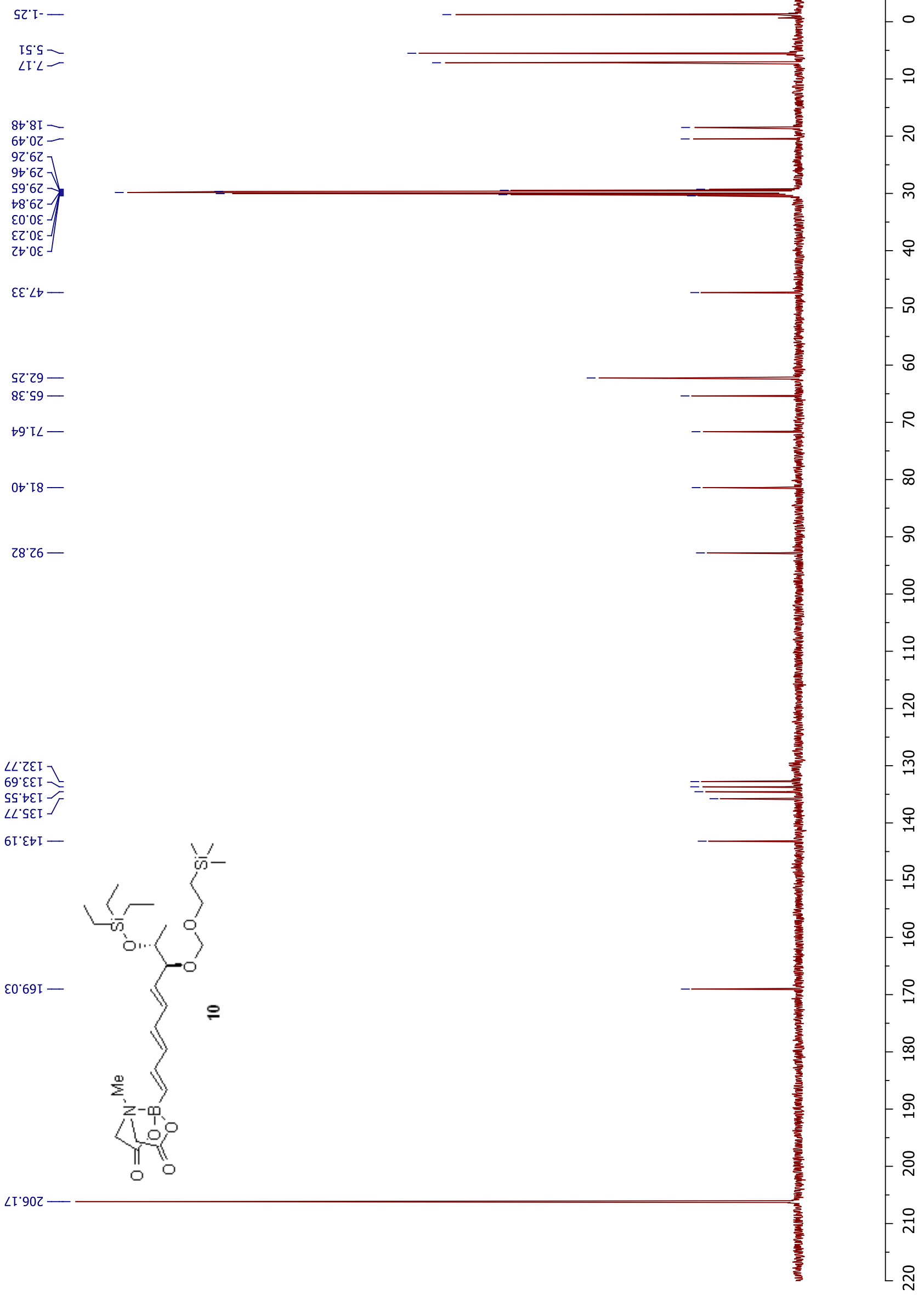




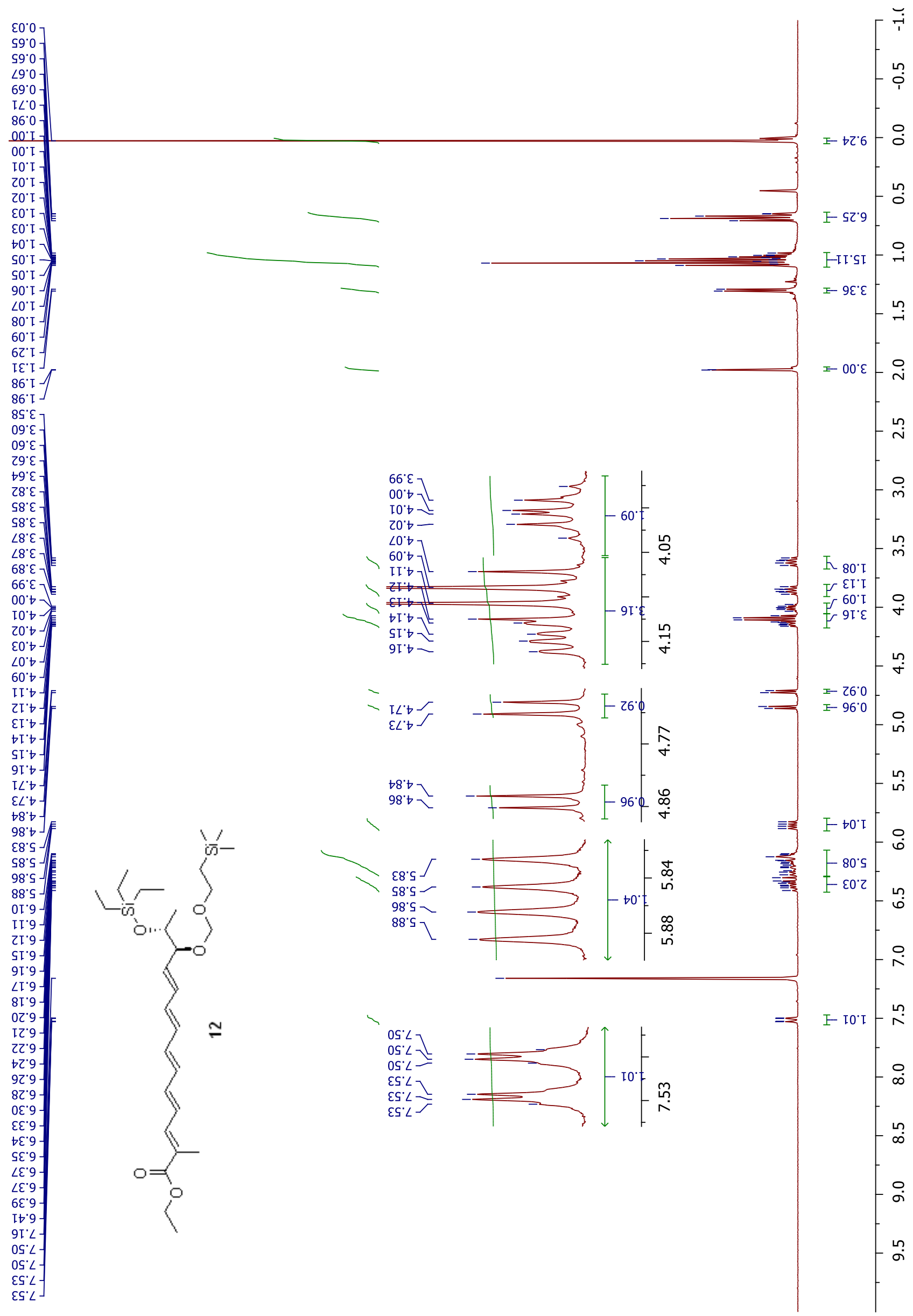




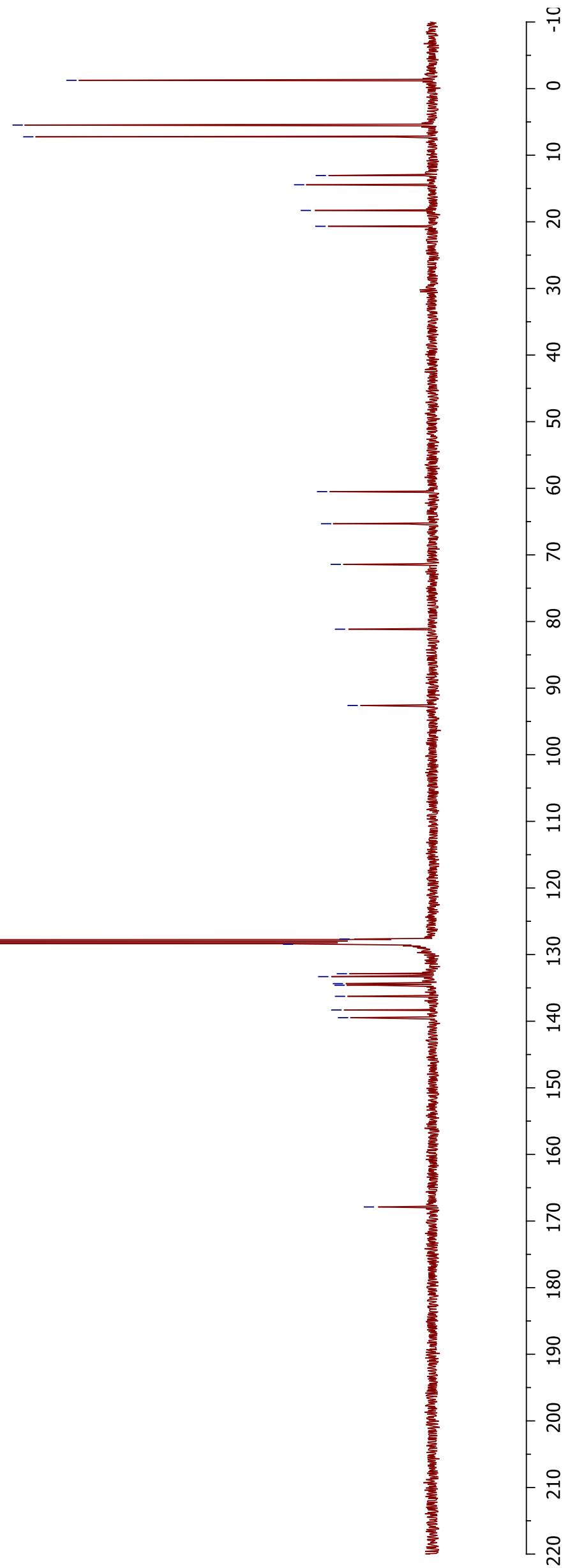




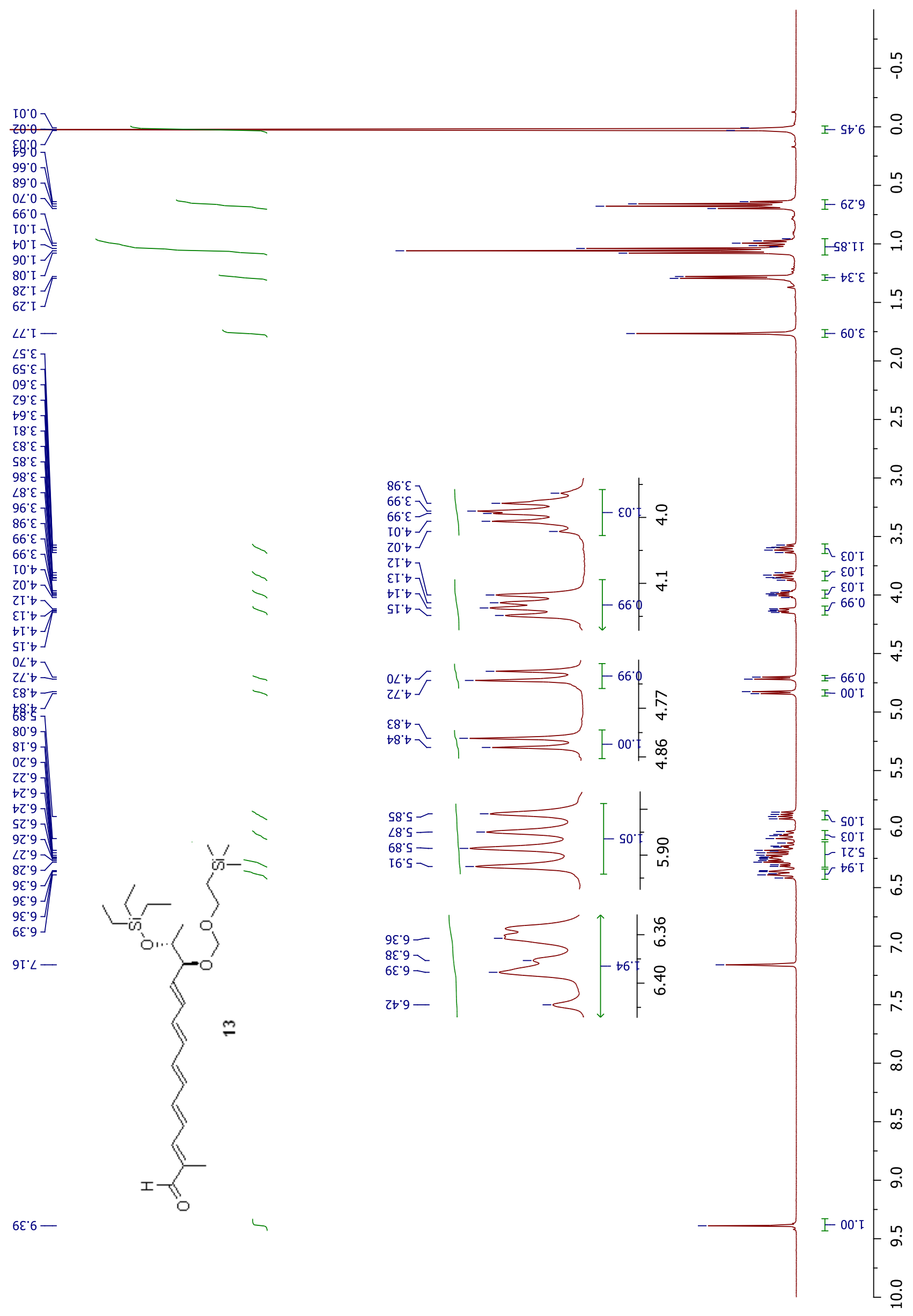




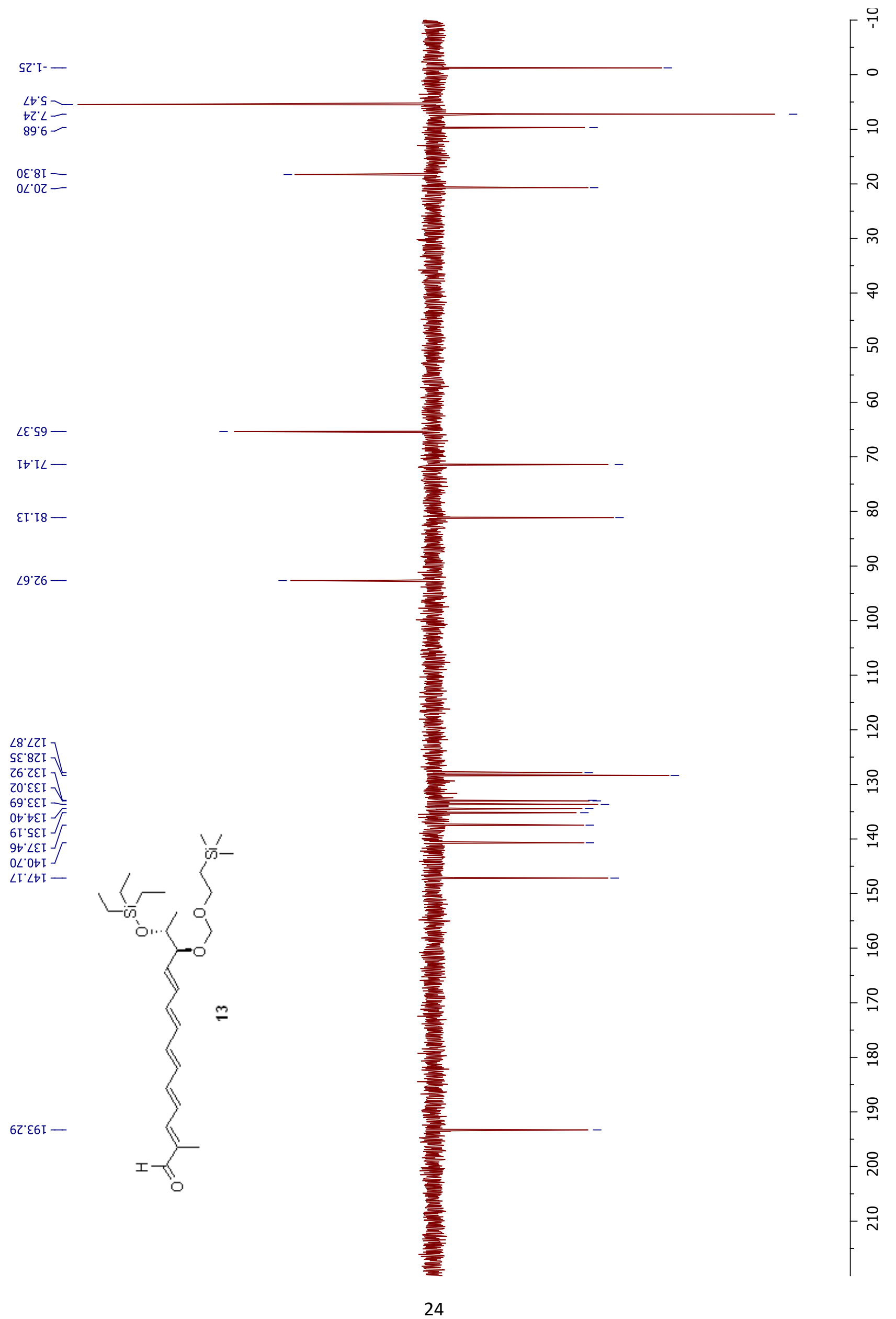



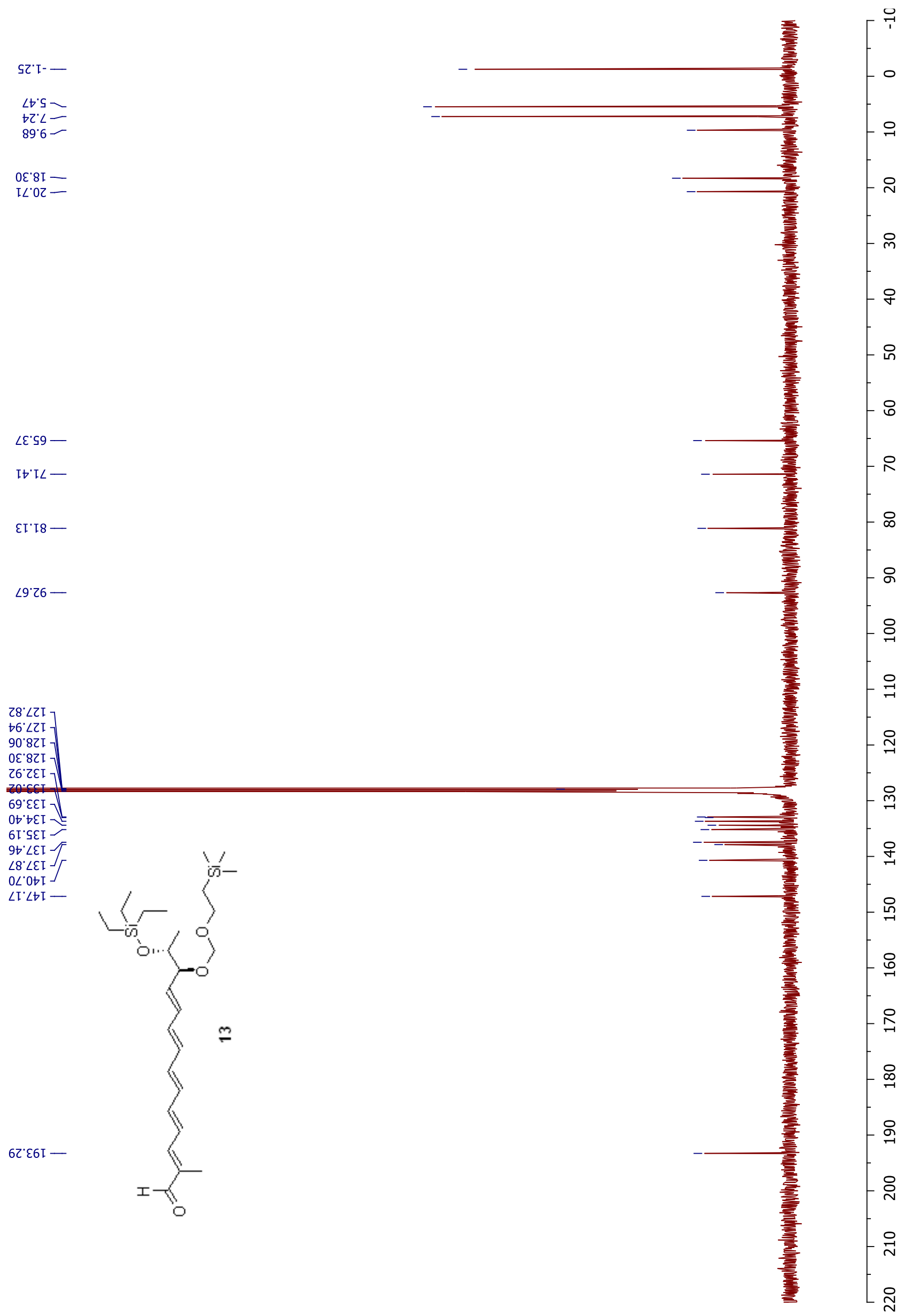


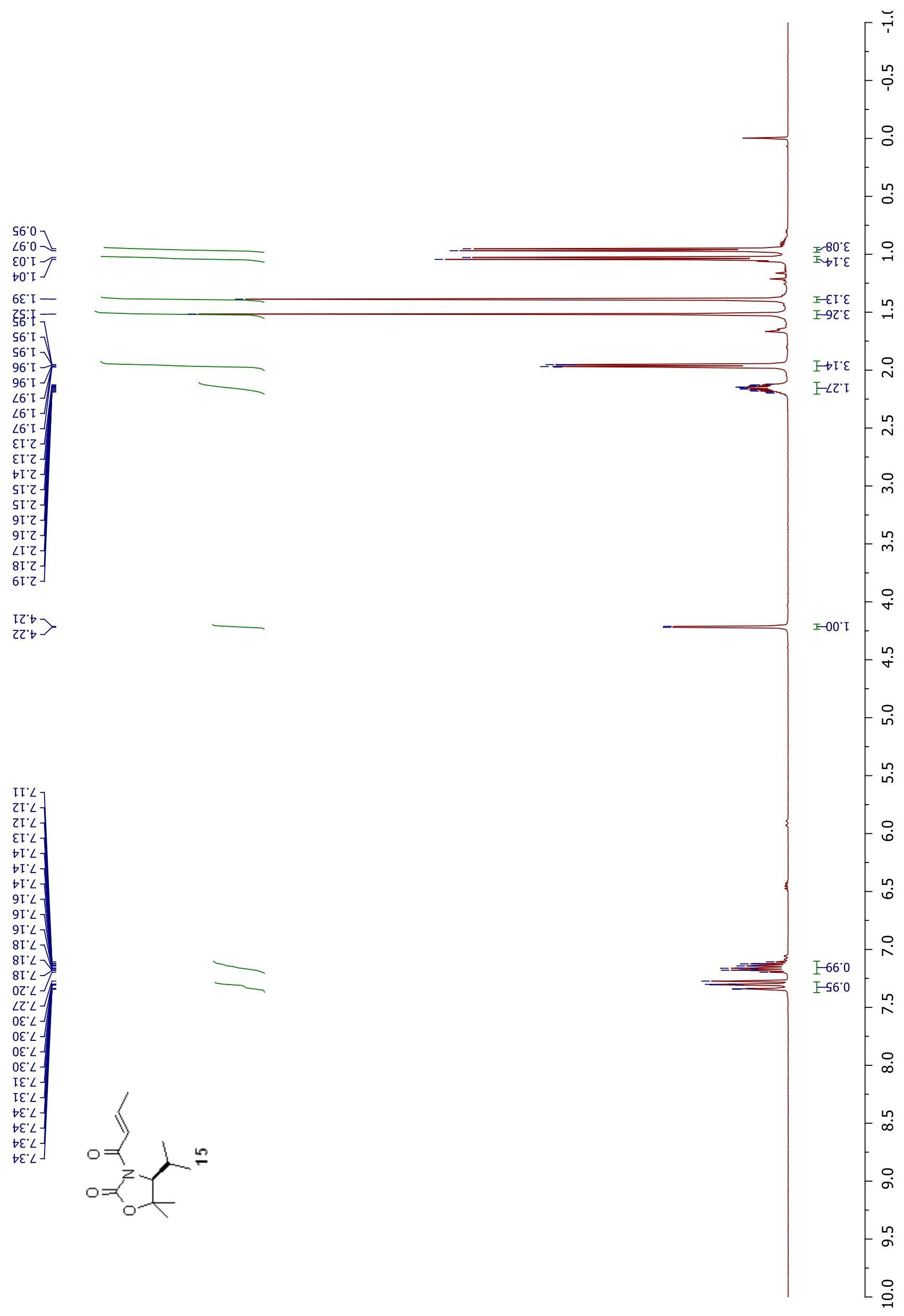


$\left.22^{\circ} \angle I\right]$

2S. I2

$\left.\begin{array}{l}2 S^{\prime} I 乙 \\ 9 S^{\prime} I 乙\end{array}\right]$

$26.82=$
08.62

$25.99-$

$78.9 L$

$9 I^{\circ} \angle L-\bar{\gamma}$

$86^{\circ} \angle L$

$98^{\prime} 28$

SO'ZZI -

$\varepsilon L ' 9 b I-$

IL'ESI -

8L'ง9เ -

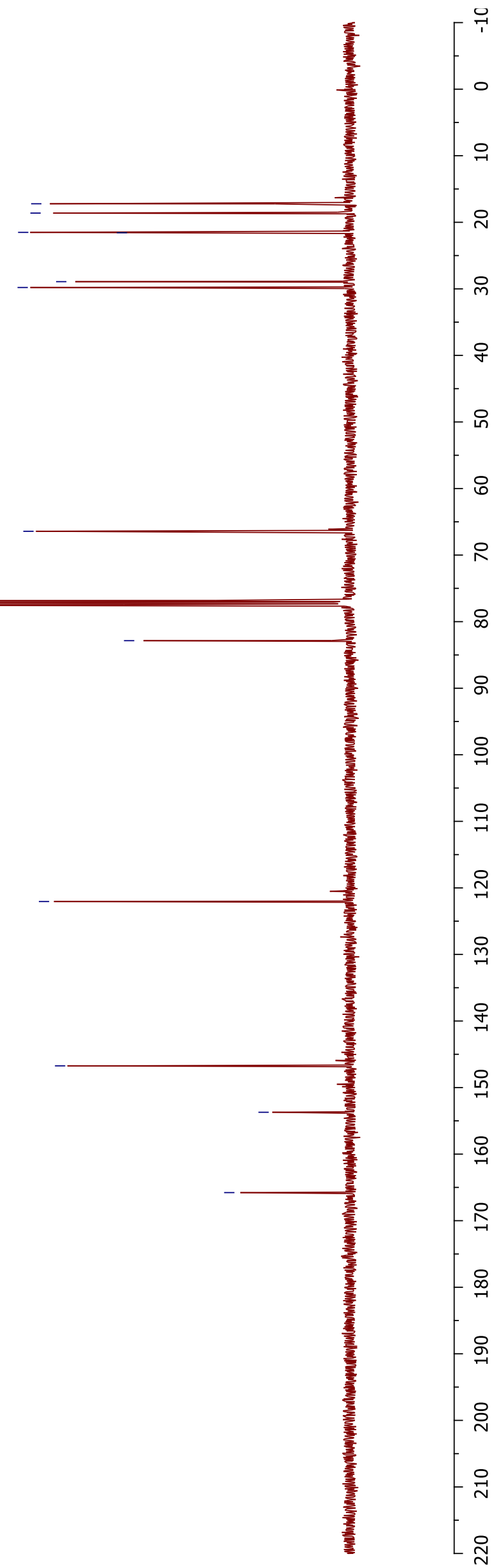




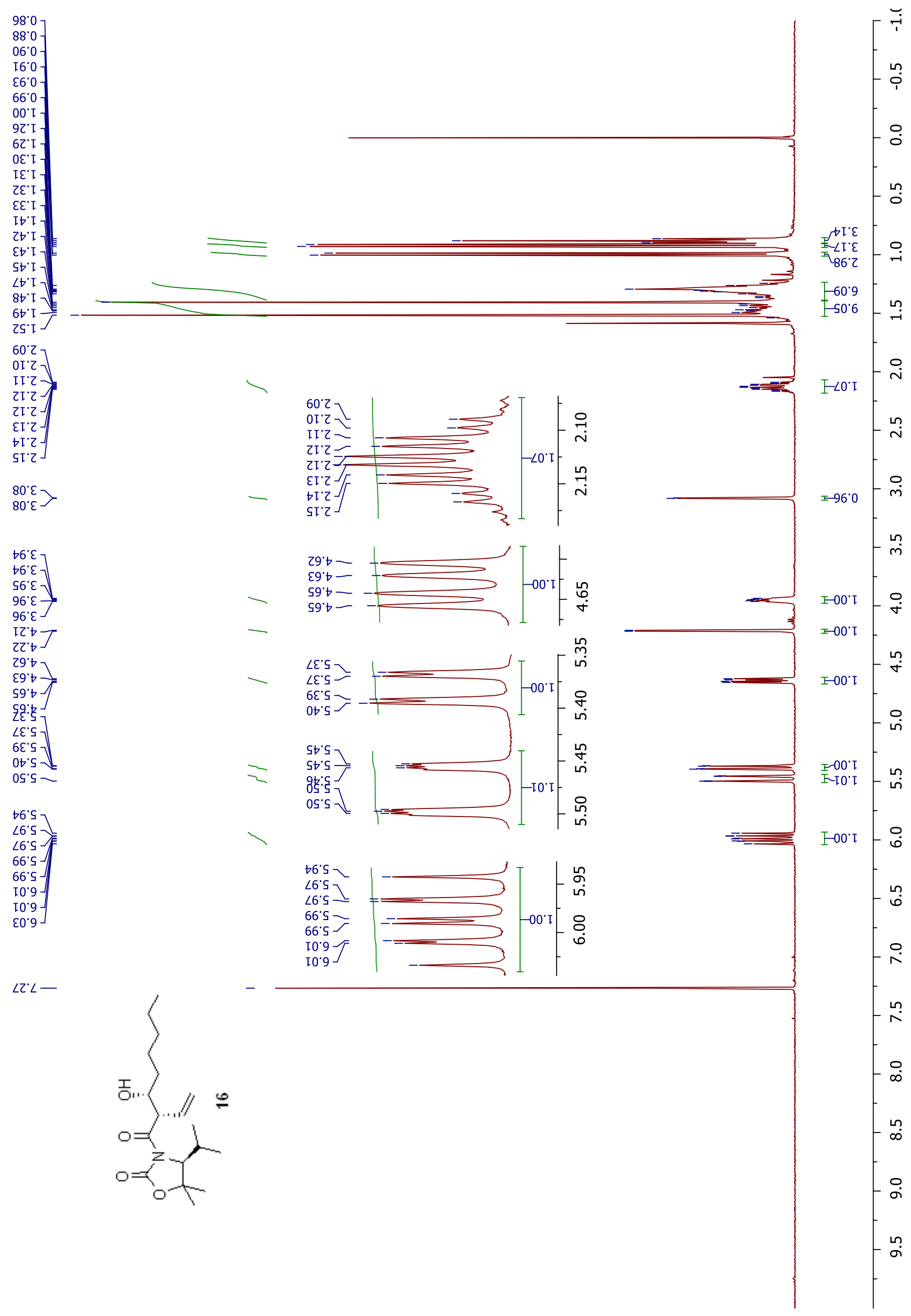




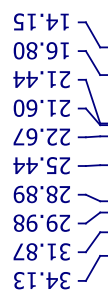

OZ'ZS -

ZI'99-

$\forall L ' T L$

$\downarrow 8^{\circ} 9 L$

$9 I^{\prime} \angle L T$

$86^{\circ} \mathrm{LL}$
$58^{\circ} \mathrm{Z}$

98. TEI -

SI'ESI -

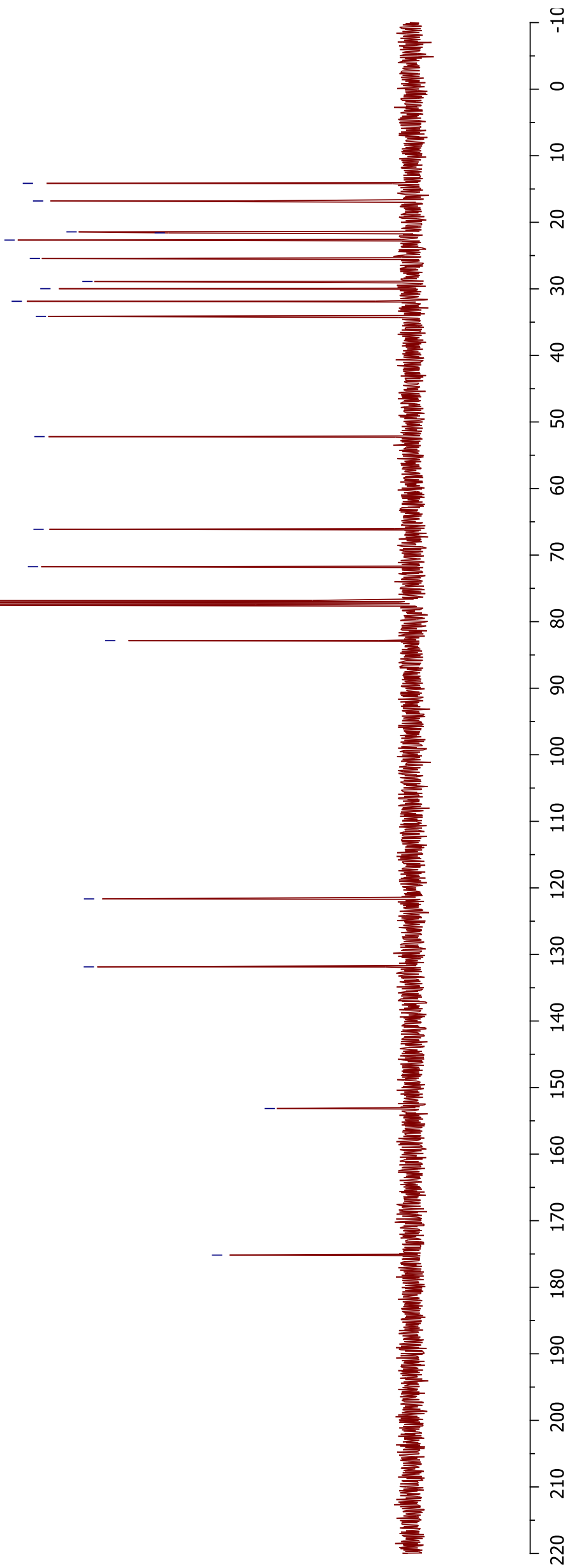




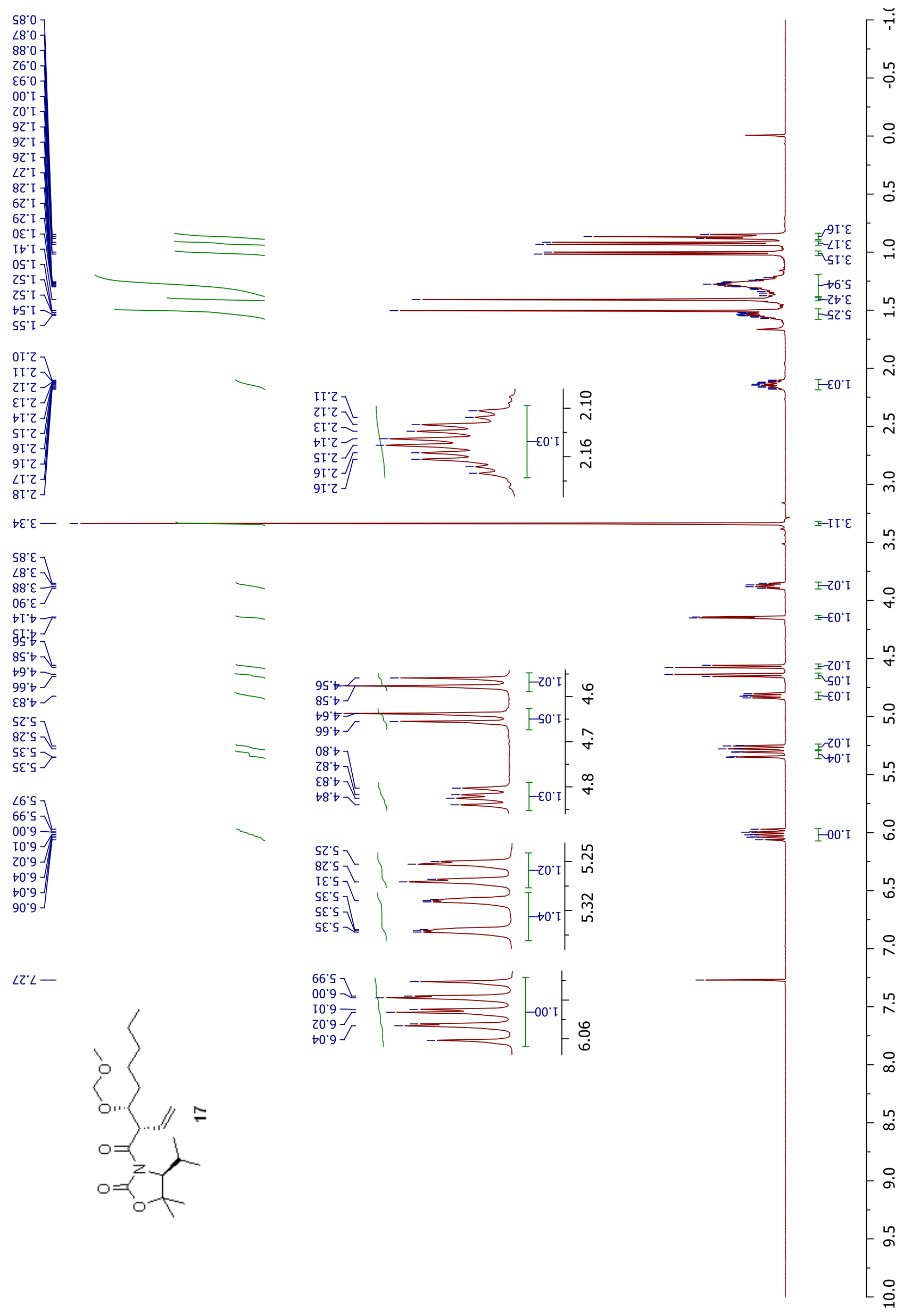




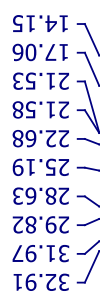

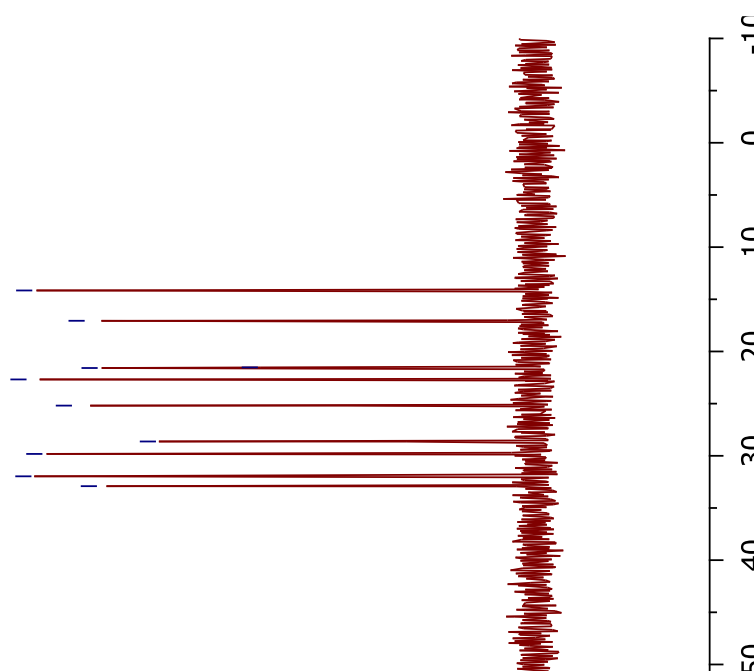

9S' IS -

80.95 -

$\angle 9^{\circ} 99$

$\dashv 8.9 L$

9I' $\angle L \frac{J}{5}$

$0 b^{\circ} 6 L$

$8[28$

$\angle S^{\prime} 96-$

$89^{\circ} 6$ I I -

96'દદ -

6t'ESI -

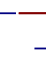


$58^{\circ} 0$

$\angle 8^{\circ} 0$

$96^{\circ} 0$

860

$\rightarrow 0^{\circ} \mathrm{I}$

ZZ' I

$\varepsilon \tau^{\prime} T$

$\nabla Z^{\prime} I$

92 '

$82^{\prime} \mathrm{T}$

OE' T

โย'[]

I $\varepsilon^{\prime}[]$

ธE' I

$\angle \varepsilon^{\circ}[-$

$8 \varepsilon^{\circ}[$

I ${ }^{\prime}$.

Et. I

St. I - I -

IS' I -

89. I

TL' $T$

IL'T

$\varepsilon[\mathrm{r}$

का' 2

9I' 2

LI'

$\angle I ' Z$

$\angle Z Z$
$8 Z^{\prime} Z$

$0 \varepsilon^{\prime} 乙$

乙ह'ح

$8 \varepsilon^{\prime} \tau-$

$8 \varepsilon ' Z$

$6 \varepsilon^{\prime} 乙$

०॰ट

故

th 2

でて

St

$\angle \varepsilon^{\prime} \varepsilon$

$\angle 0^{\circ} \circ$

$80^{\circ} \mathrm{t}$

60.5

OI'

II't

ZI'b

$\varepsilon I^{\prime} b$

SI'b

$\angle b^{\circ} t$

$6 b^{\circ} \downarrow-$

$6 b^{\circ} t$

OS' $\Rightarrow$

IS' $b$

IS'

$\angle 9^{\circ} \mathrm{t}$

$69^{\circ} \circ$

SI' $S$

SI'S

9I'S

6I'S

$6 I^{\prime} S$

เ8. $\mathrm{s}$

$98^{\prime} \mathrm{S}$

$88^{\circ} \mathrm{S}$

$68^{\circ} \mathrm{s}$

$06^{\circ} \mathrm{S}$

I6. $\mathrm{S}$

\&6. $\mathrm{S}$
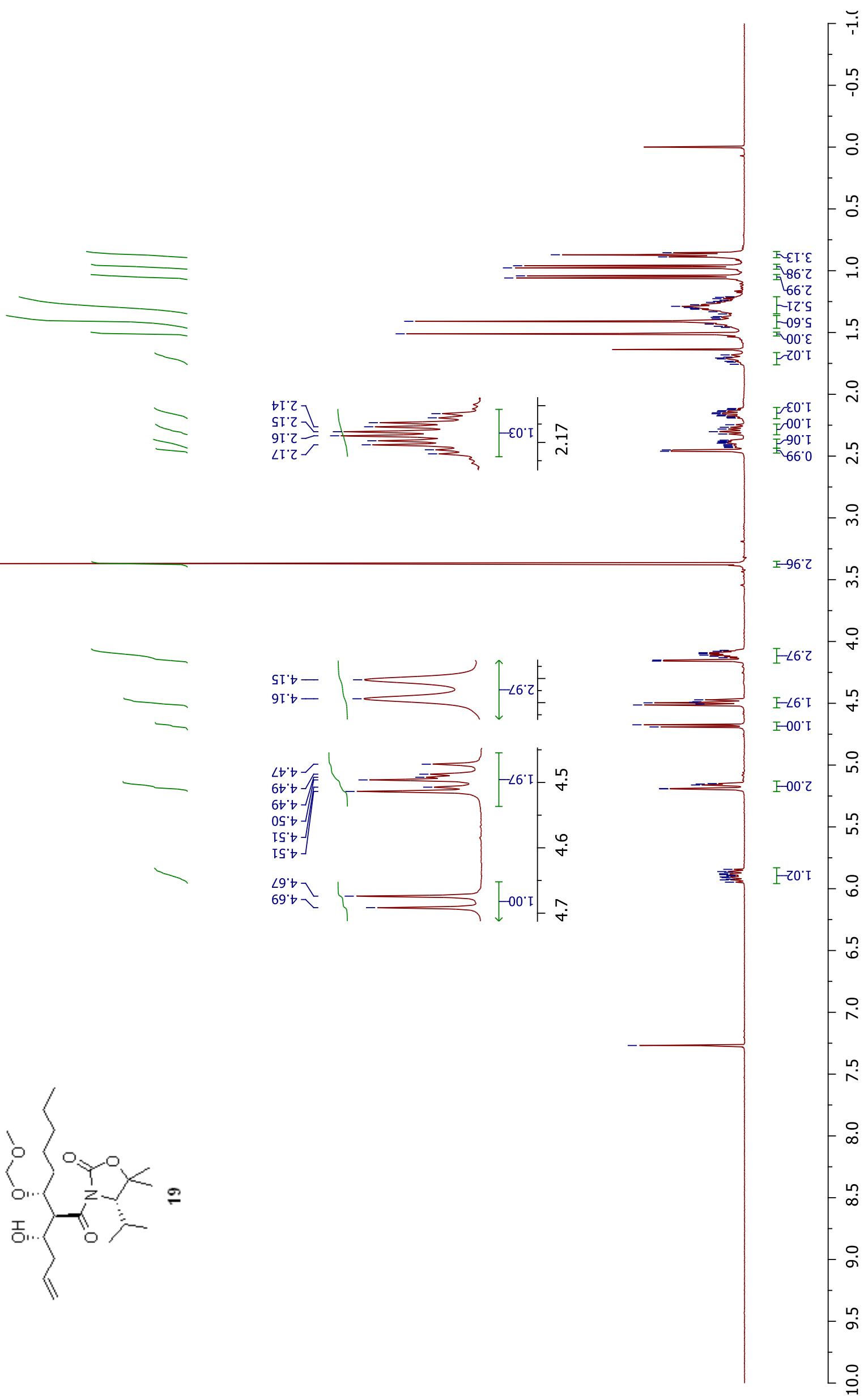


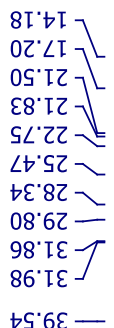

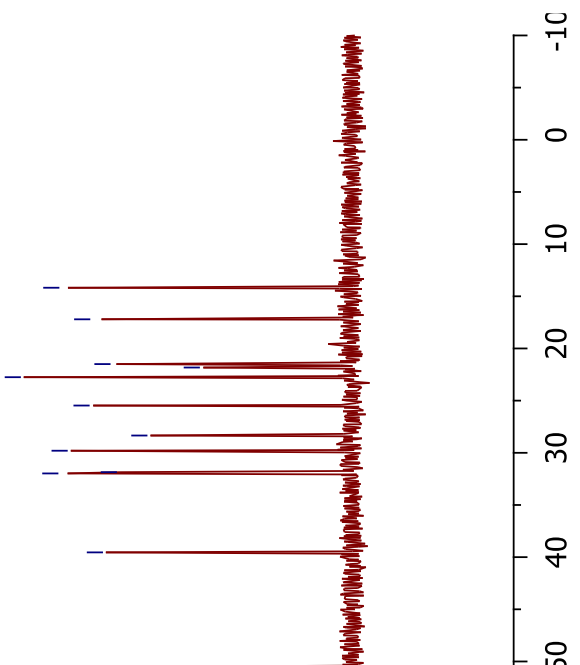

IS.0S -

sZ'9s -

$b Z^{\prime} \angle 9-$

$\angle I^{\prime} O L$

$\succ 8.9<>$

$9{ }^{\circ} \mathrm{LL}$

ว०' 8 -

6I'96-

68.8 I I -

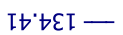

$\forall 0^{\circ} t S I-$

$06^{\circ} Z \angle I-$<smiles>C=CCCC(CCOC)C(=O)C1=C(C(C)C)C(C)(C)OC1=O</smiles>

음

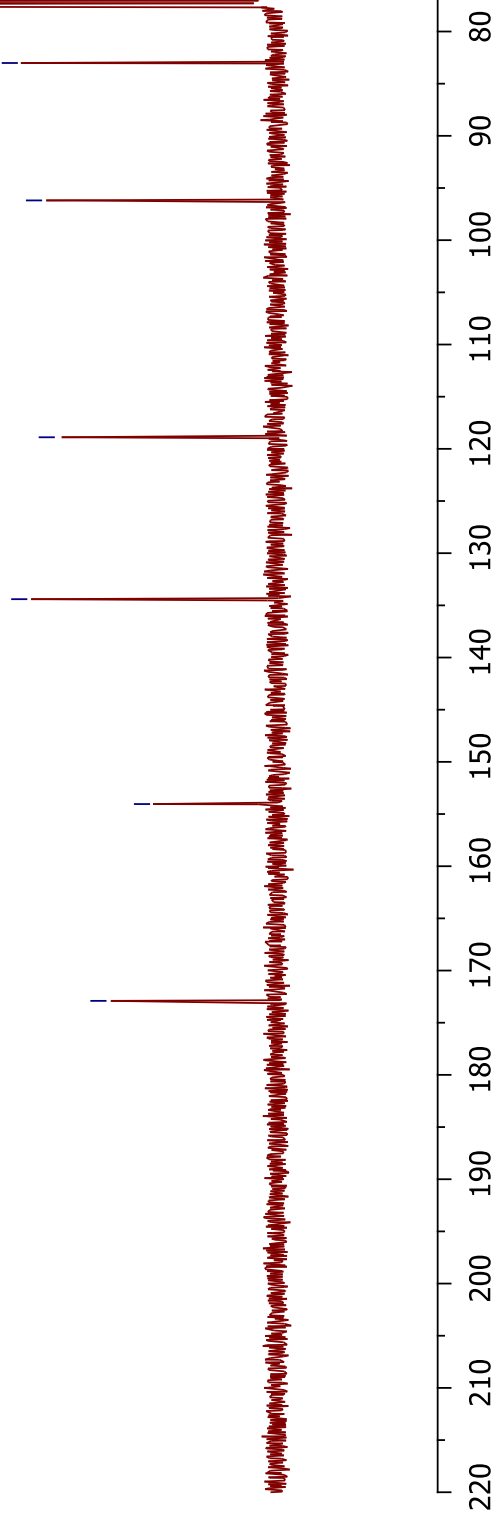




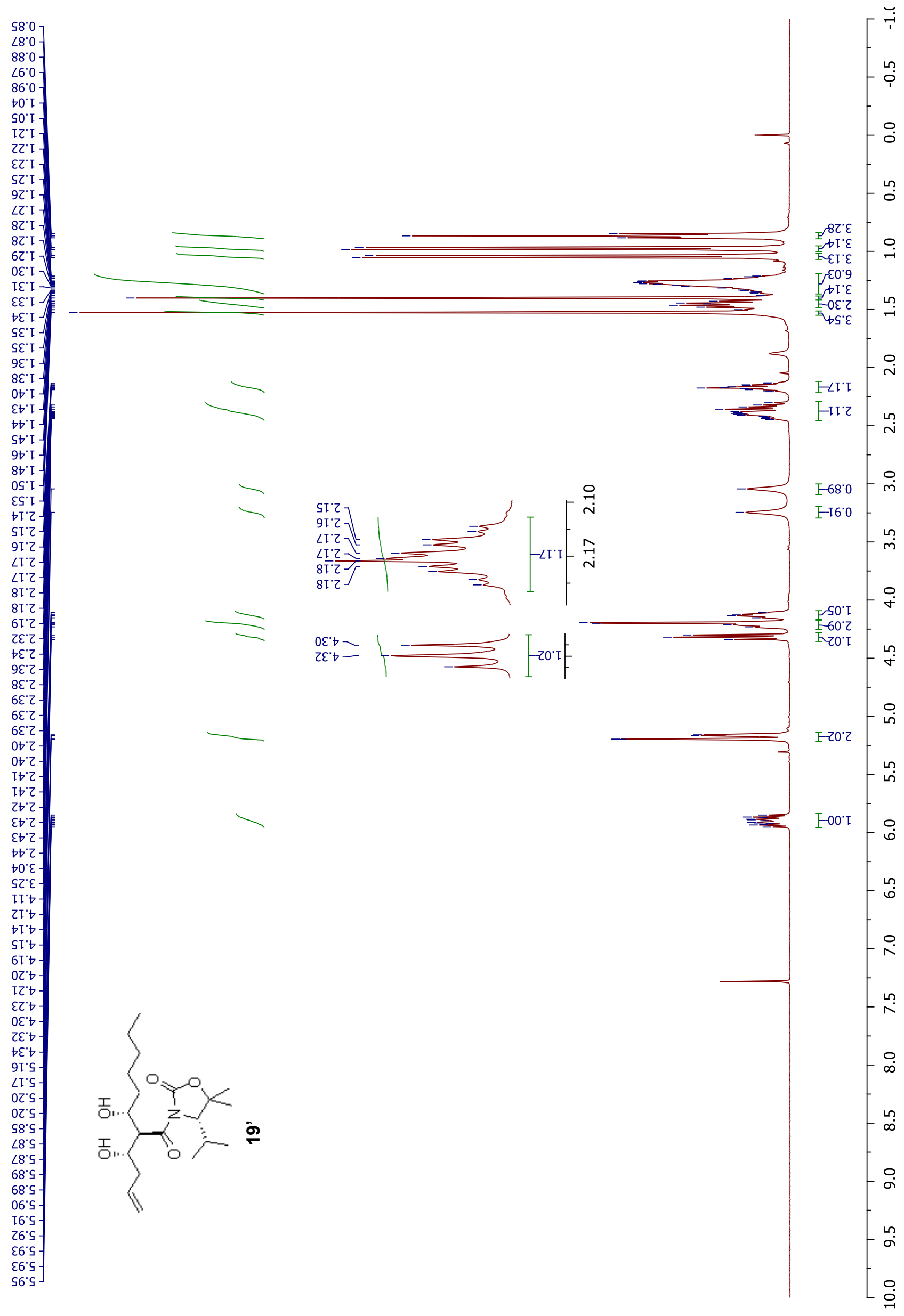


$\varepsilon \tau^{\prime} \downarrow \tau$

$90 \angle \mathrm{I} 工$

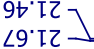

69.22

$\angle \mathrm{CZ}$

$\angle L \cdot 8 z-T$

$\angle 8.62$ 工

โ8' IE

$\rightarrow 8^{\circ} \downarrow \varepsilon$

$8 L^{\prime} 6 \varepsilon-$

โธ'ยS -

ह8: है: -

$\left.\begin{array}{l}\succ 8^{\circ} 9 L \\ 9 I^{\circ} L L\end{array}\right]$ L

$8 b^{\circ} \angle L$

00'๕8-

$00^{\circ} 6$ II -

Z乙'†ยI -

Z0" $\mathrm{HSI}$ -
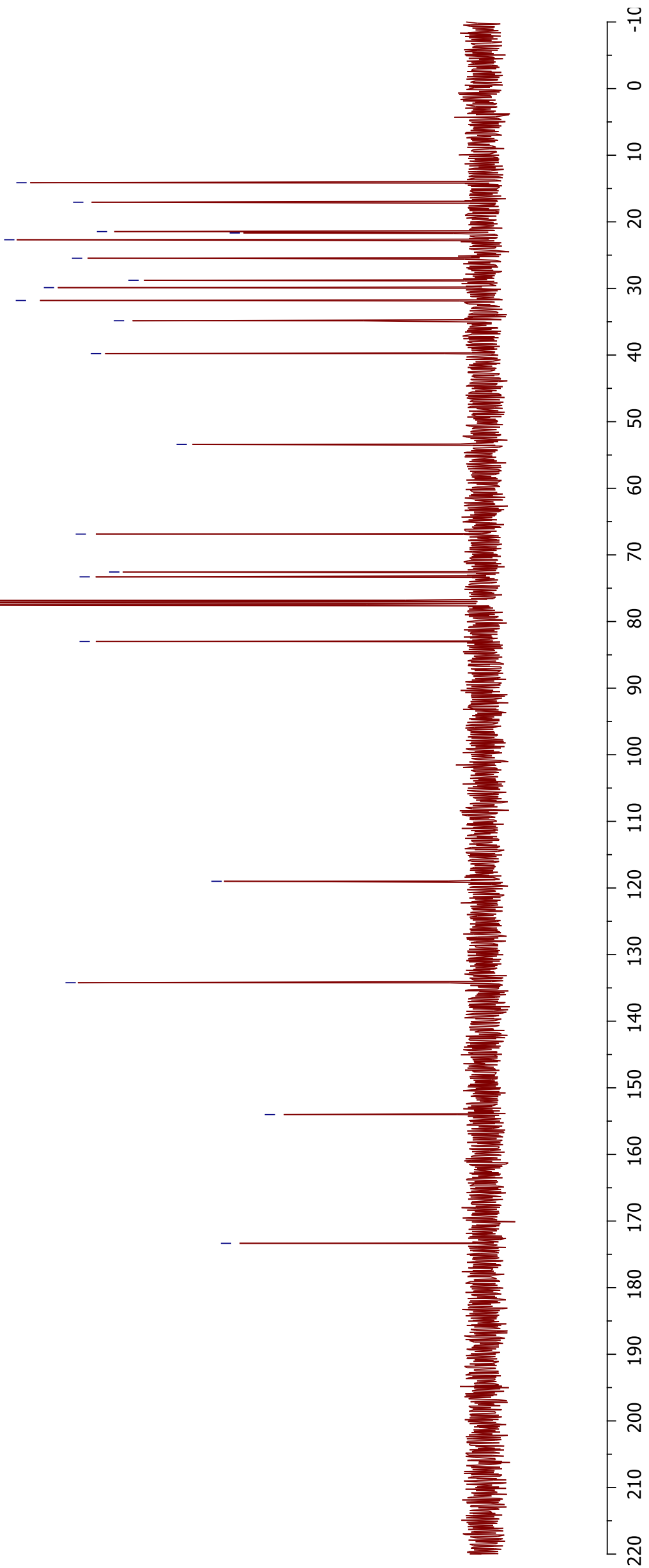


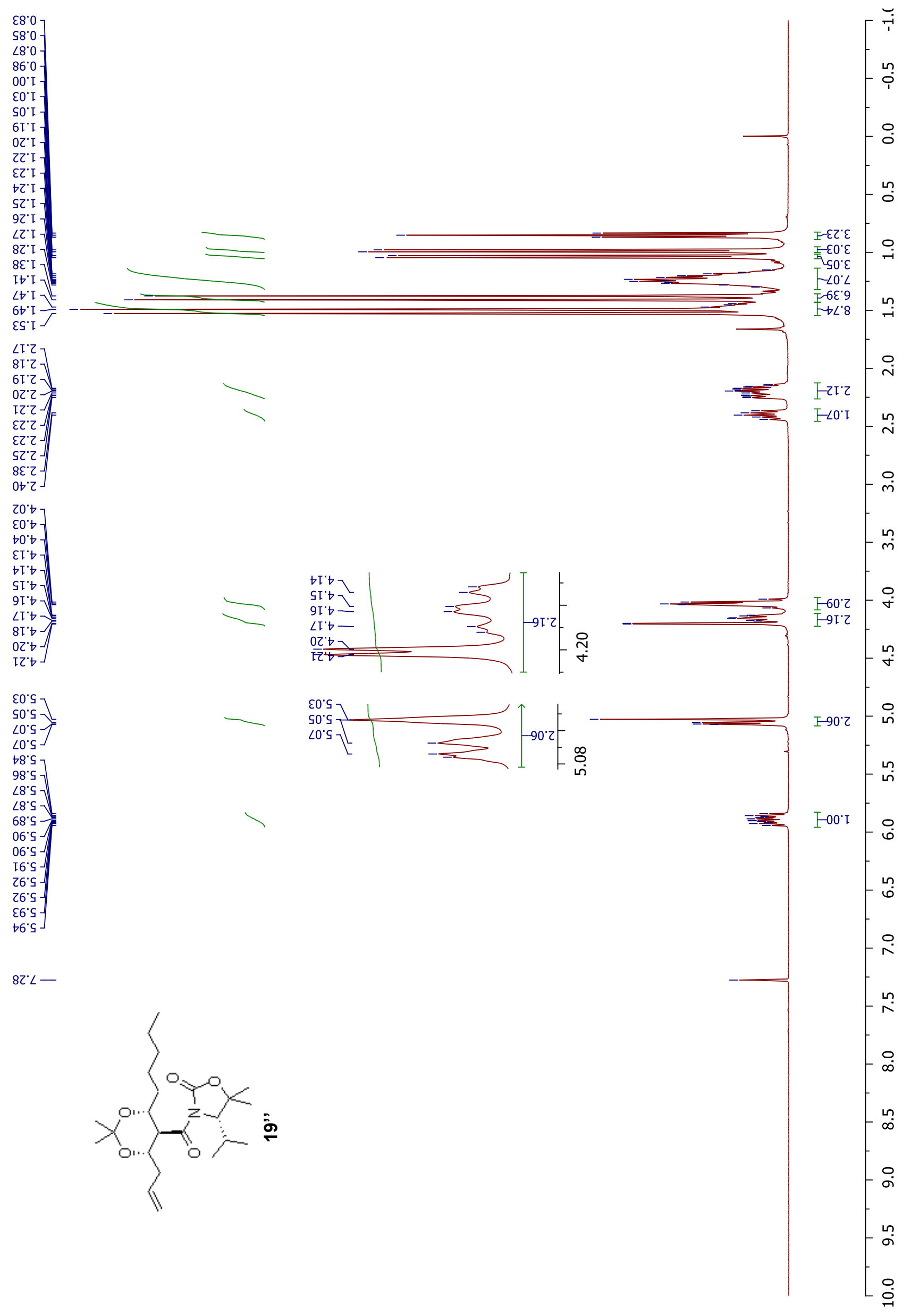




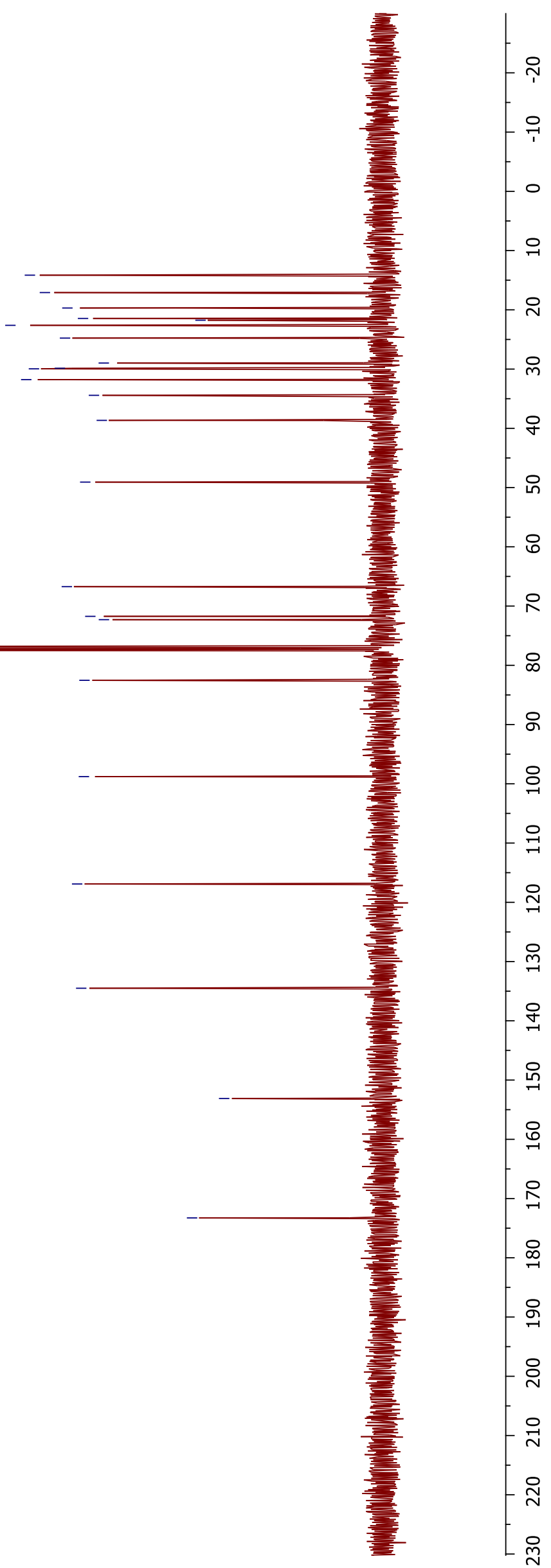




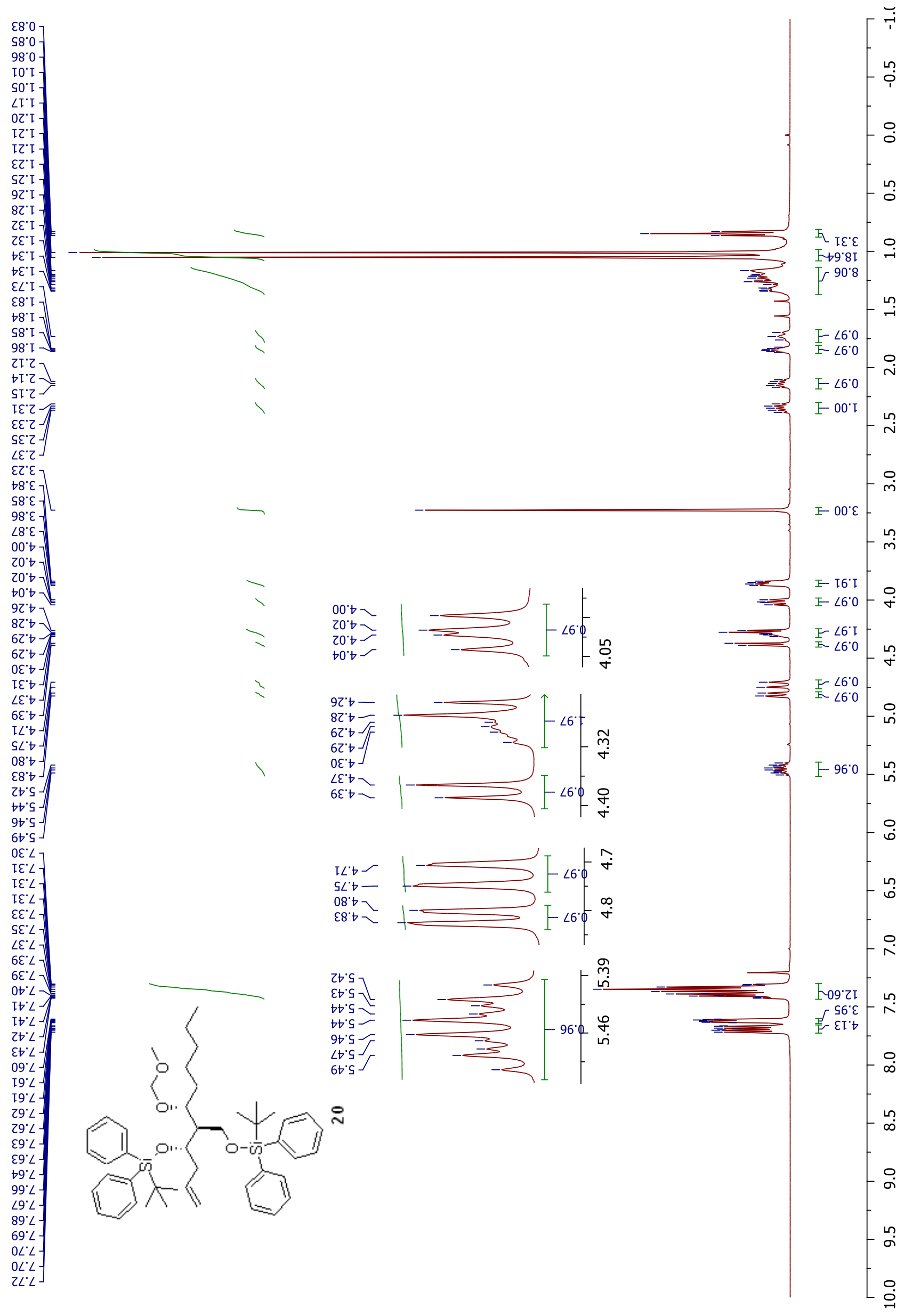




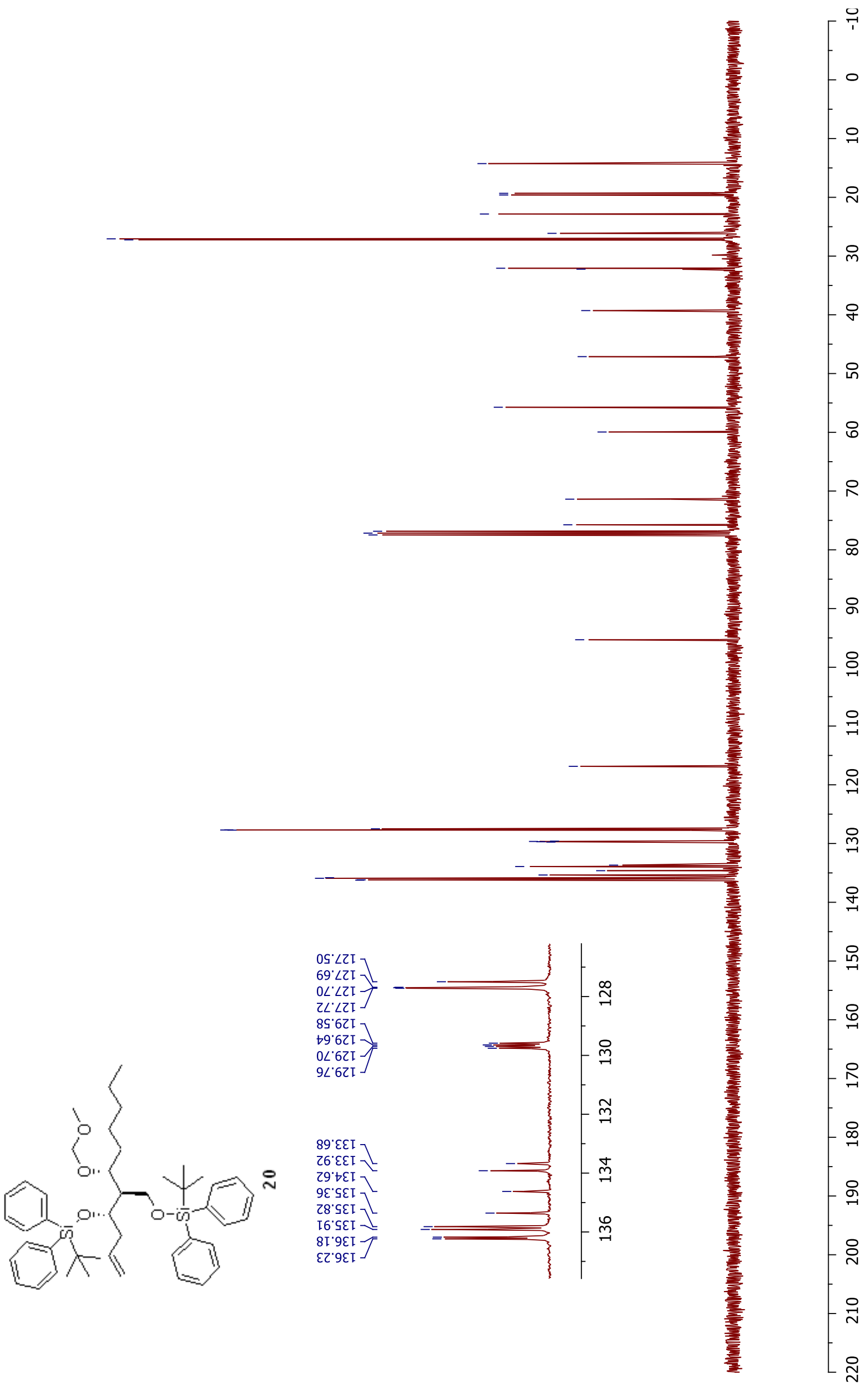




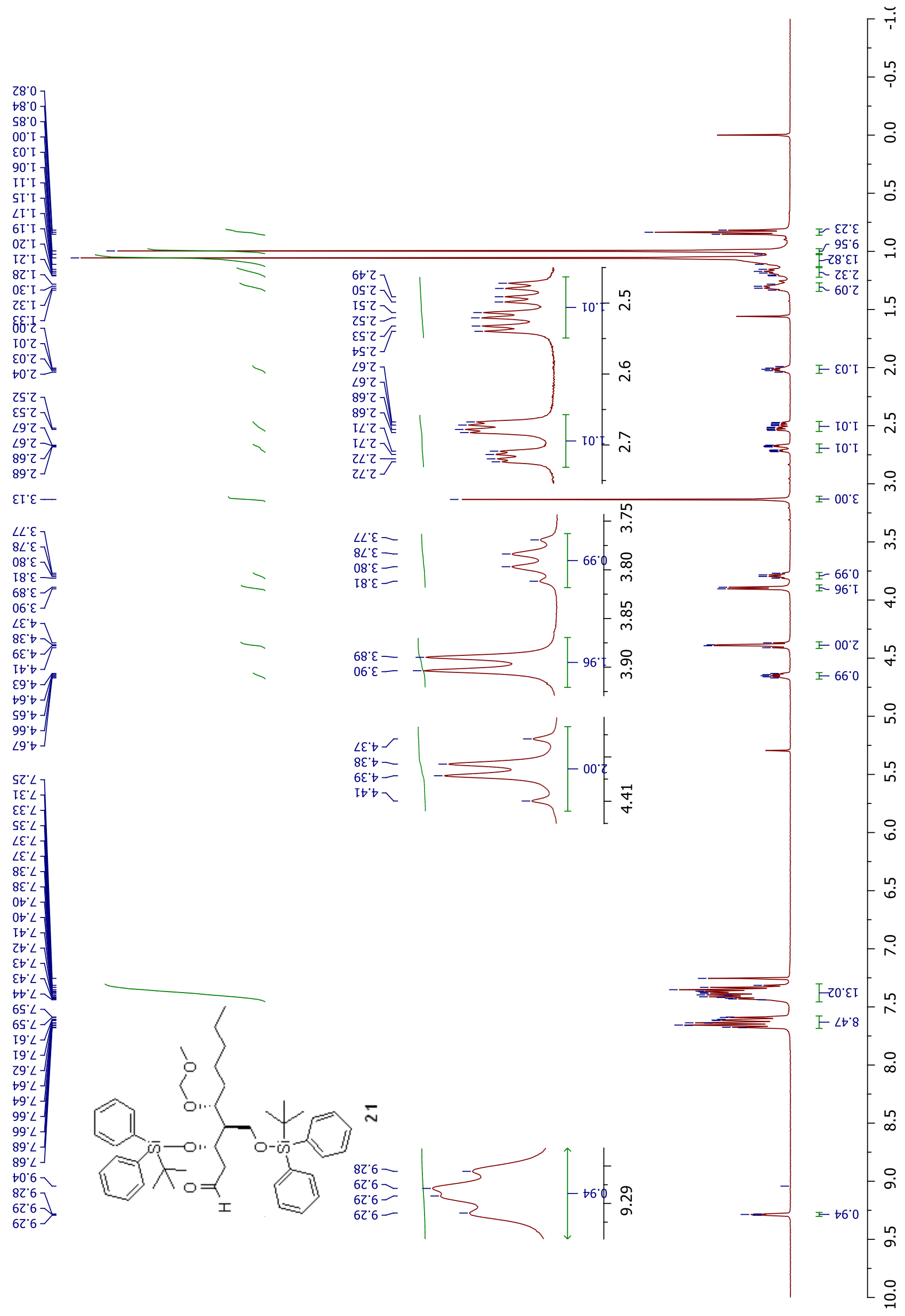


$0 Z^{\prime} \downarrow I-$

દย'6[

$\angle \varepsilon^{\prime} 6[]$

SL'ZZ

$9 I^{\circ} \mathrm{SZ}-$

II'LZ -

$86^{\circ}$ โย

20'Zع $>$

$78.8 \mathrm{t}$

$62.65-$

I8. 95

$8 L ' 65$

$\downarrow \tau: \angle 9-$

$\rightarrow S^{\prime} 9 L$

9I $\angle L-$

$\left.80^{\circ} \angle L\right]$

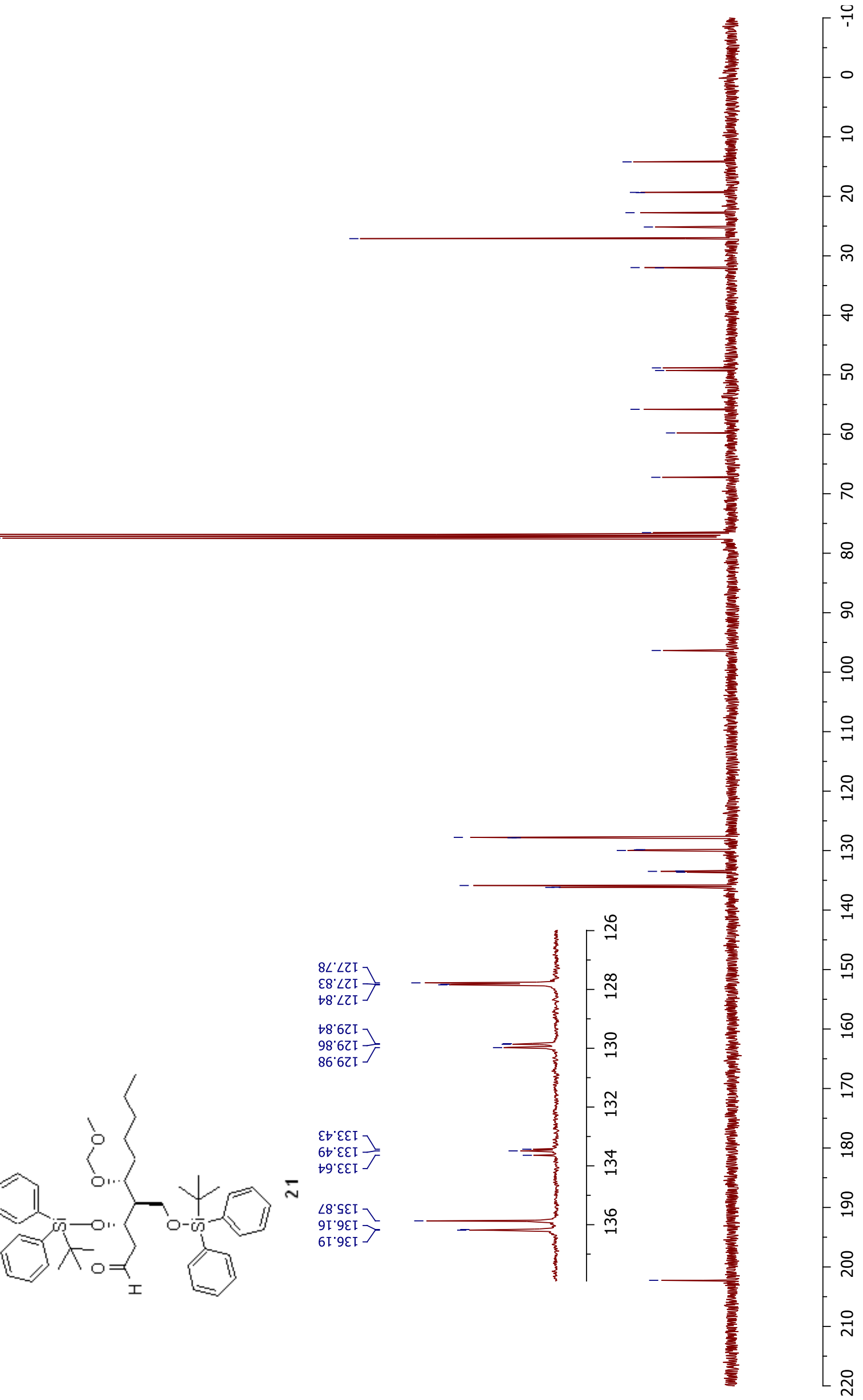

$9 \varepsilon^{\prime} 96-$

$8 L^{\circ} \angle Z I$

$\varepsilon 8^{\circ} \angle Z I$

$\checkmark 8^{\circ} \angle Z I_{-}$

$98^{\circ} 62 \mathrm{~L}$.

86.62I

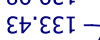

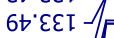

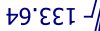

$\angle 8$ 'SE I

9โ'9ع ]

6['9ع[ ]

sz'zoz- 

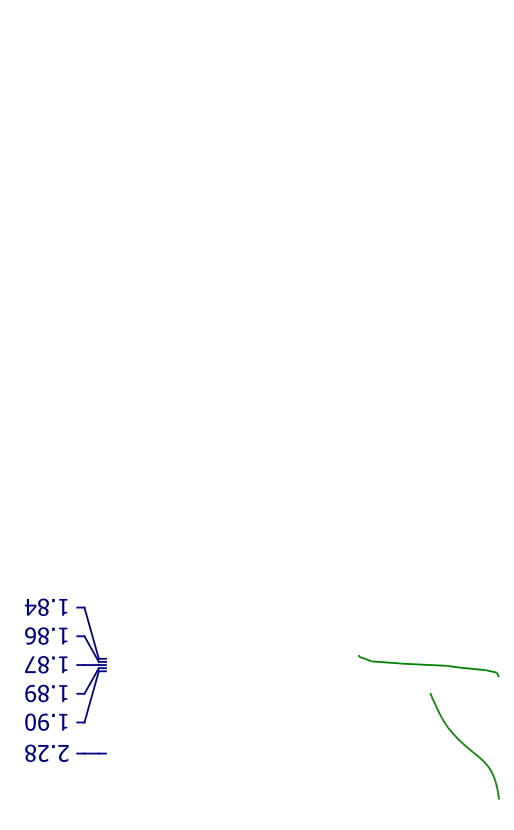

$\left.\begin{array}{l}\varsigma 9^{\circ} \varepsilon \\ \angle 9^{\circ} \varepsilon \\ 89^{\circ} \varepsilon \\ 6 L^{\circ} \varepsilon\end{array}\right]$
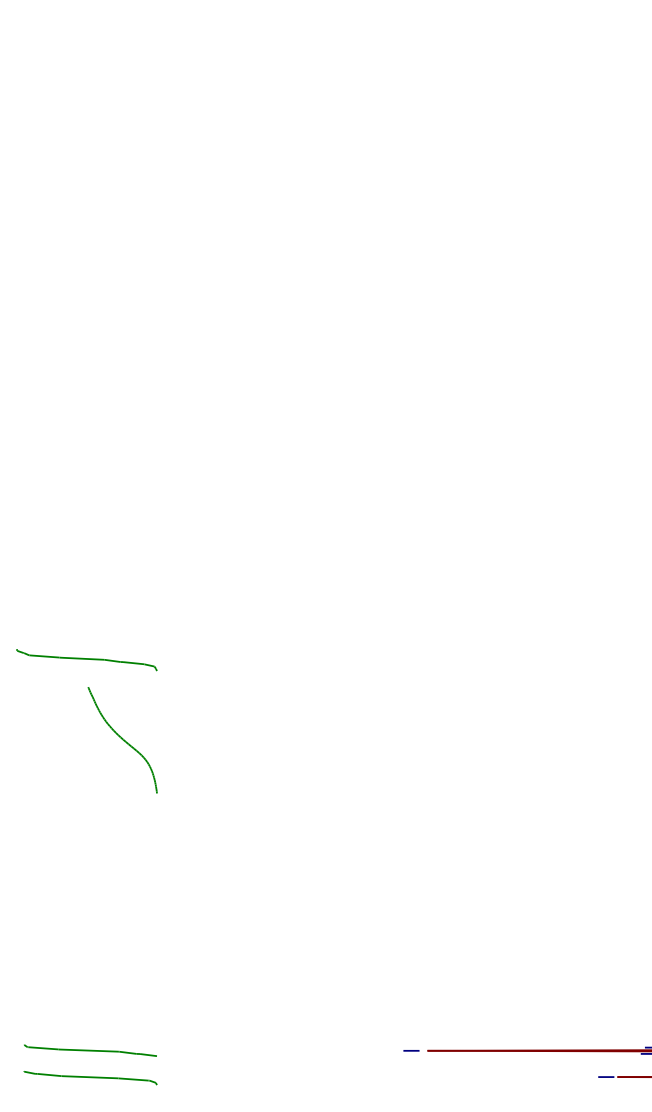

عS'† -
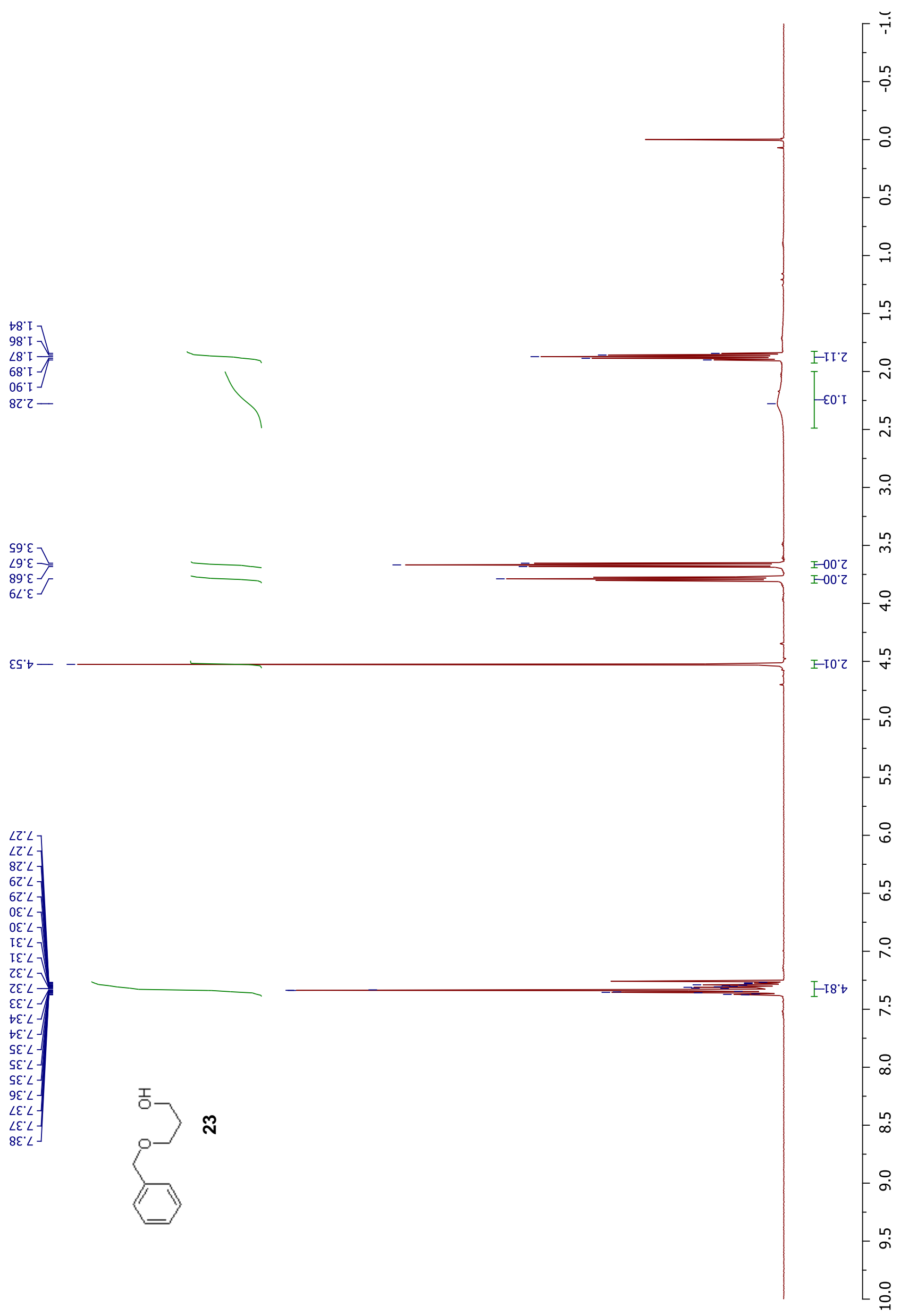


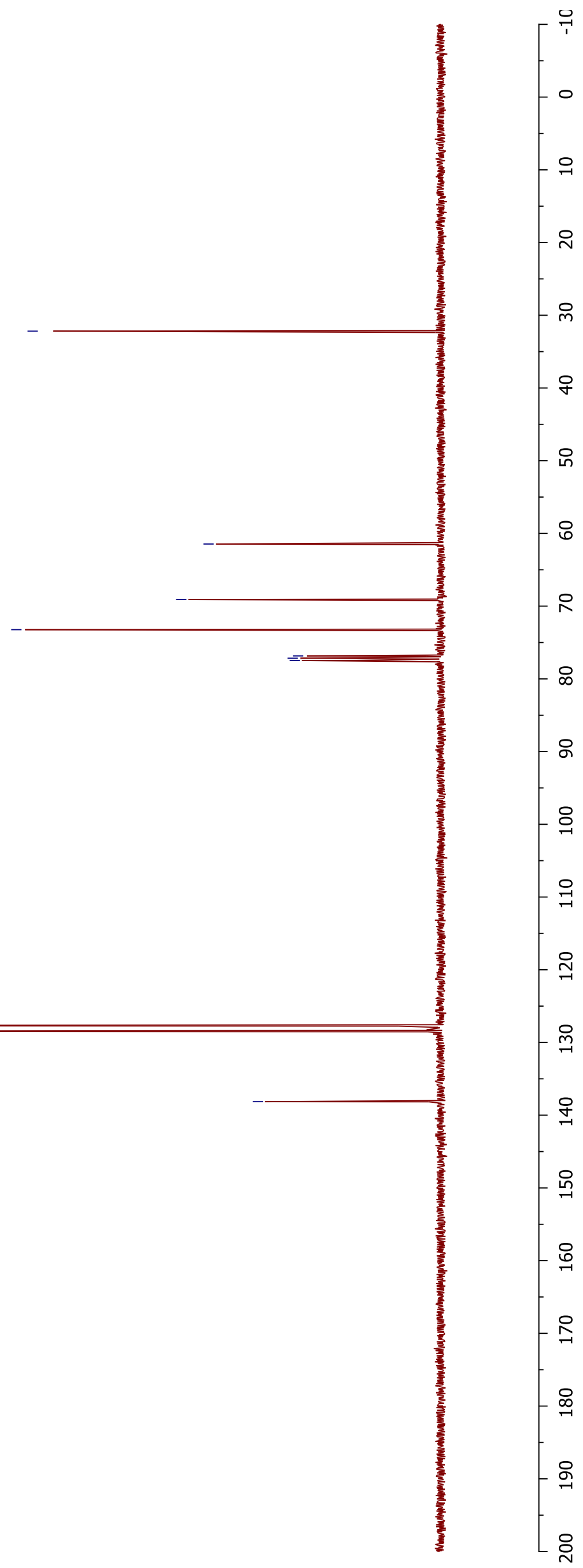

69' $\angle Z I$

${ }_{\angle L^{\prime} \angle Z I} \cdot \bar{T}$

$\downarrow I^{\circ} 8 \varepsilon \mp-$

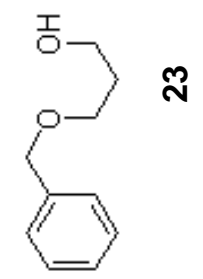



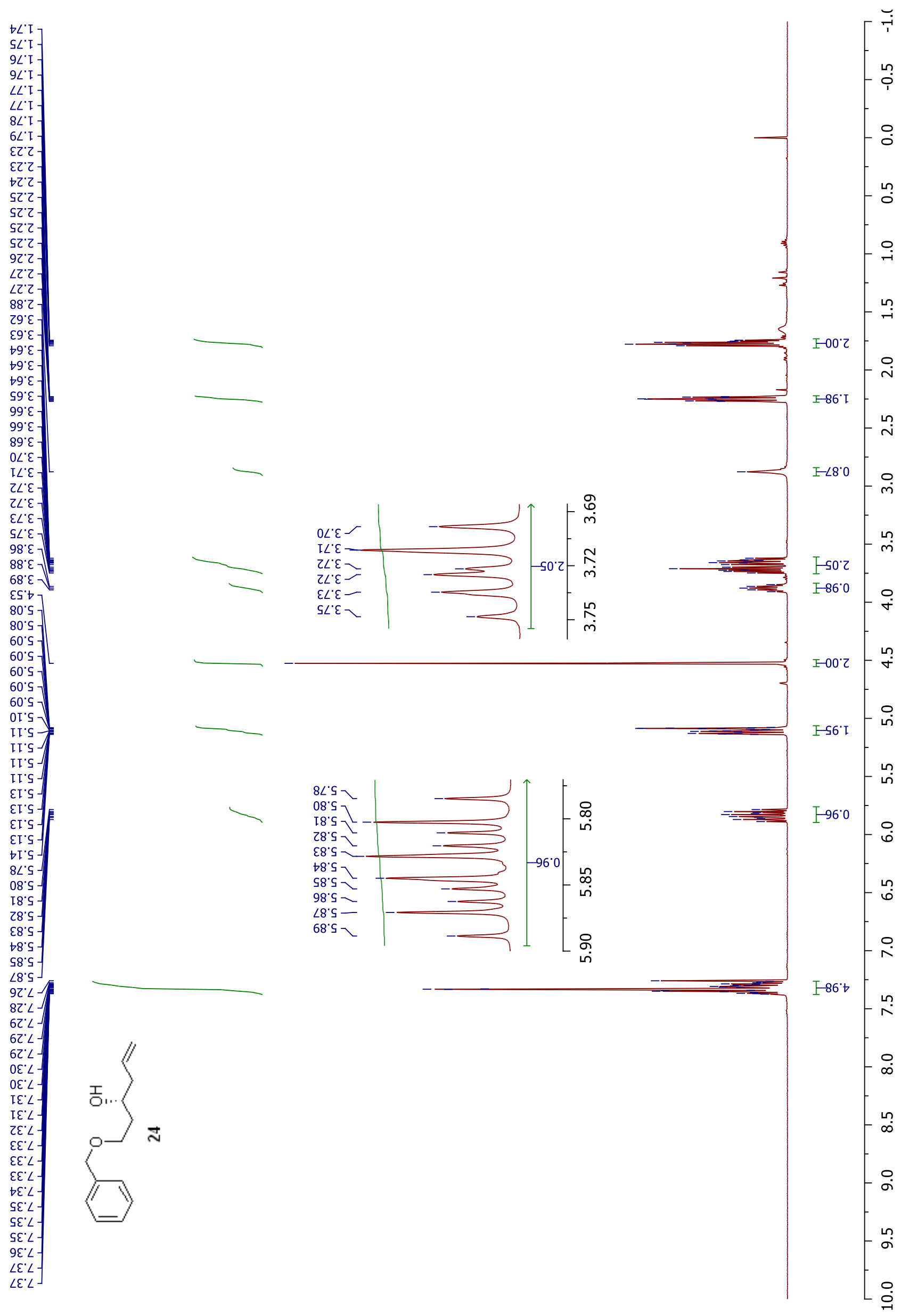


$$
\begin{aligned}
& \text { 88. } \\
& \text { } 66^{\circ} \mathrm{t} t
\end{aligned}
$$

86.89

$05^{\circ} 0<-$

$\varepsilon \varepsilon ' \varepsilon L>$

to $\angle L>$

†E'LL]

$09^{\circ} \angle I I-$

$69^{\circ} \angle Z I$

$9 \angle 2 Z T]$

$\angle D^{\circ} 8 Z \mathrm{I}$

$68^{\circ} \mathrm{HEI}$ -

$96^{\circ} \angle E I-$

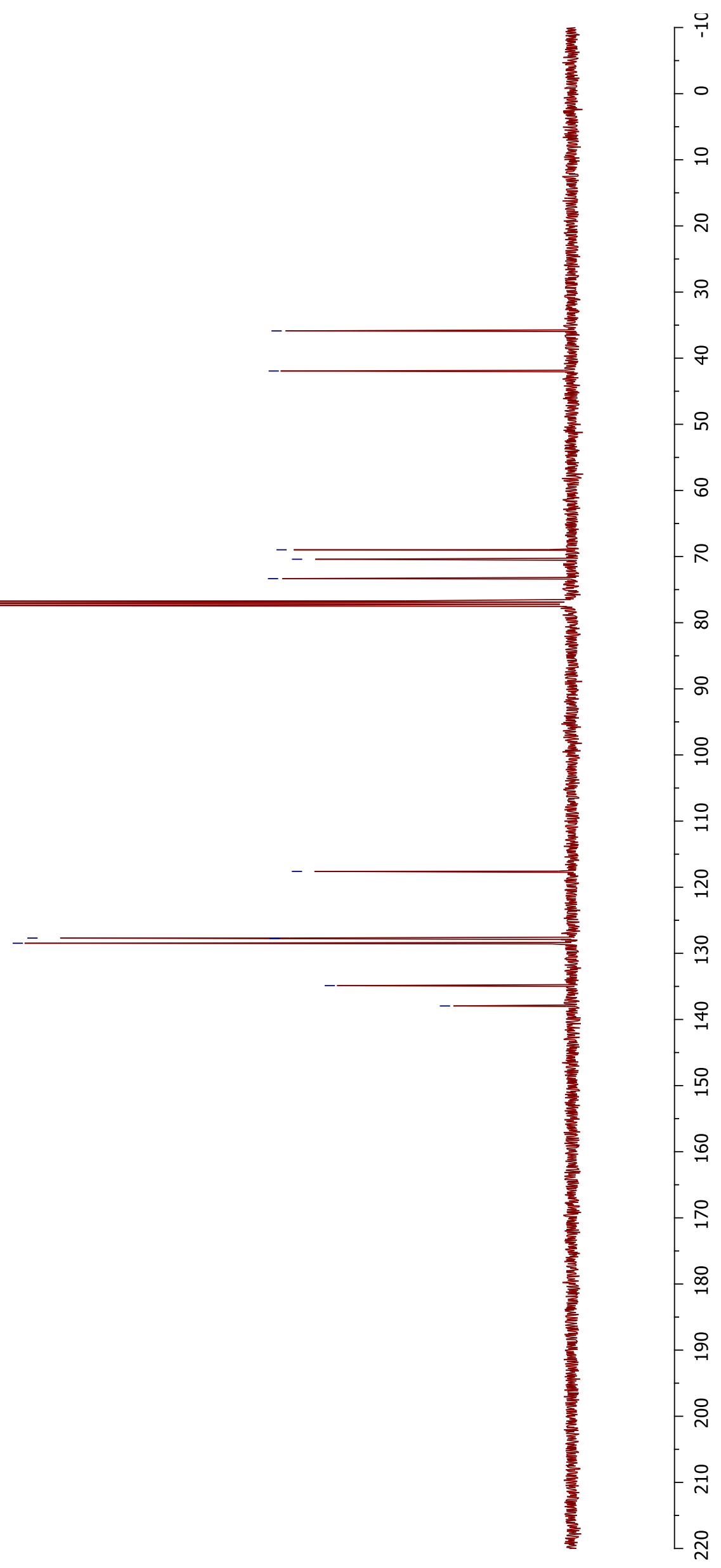




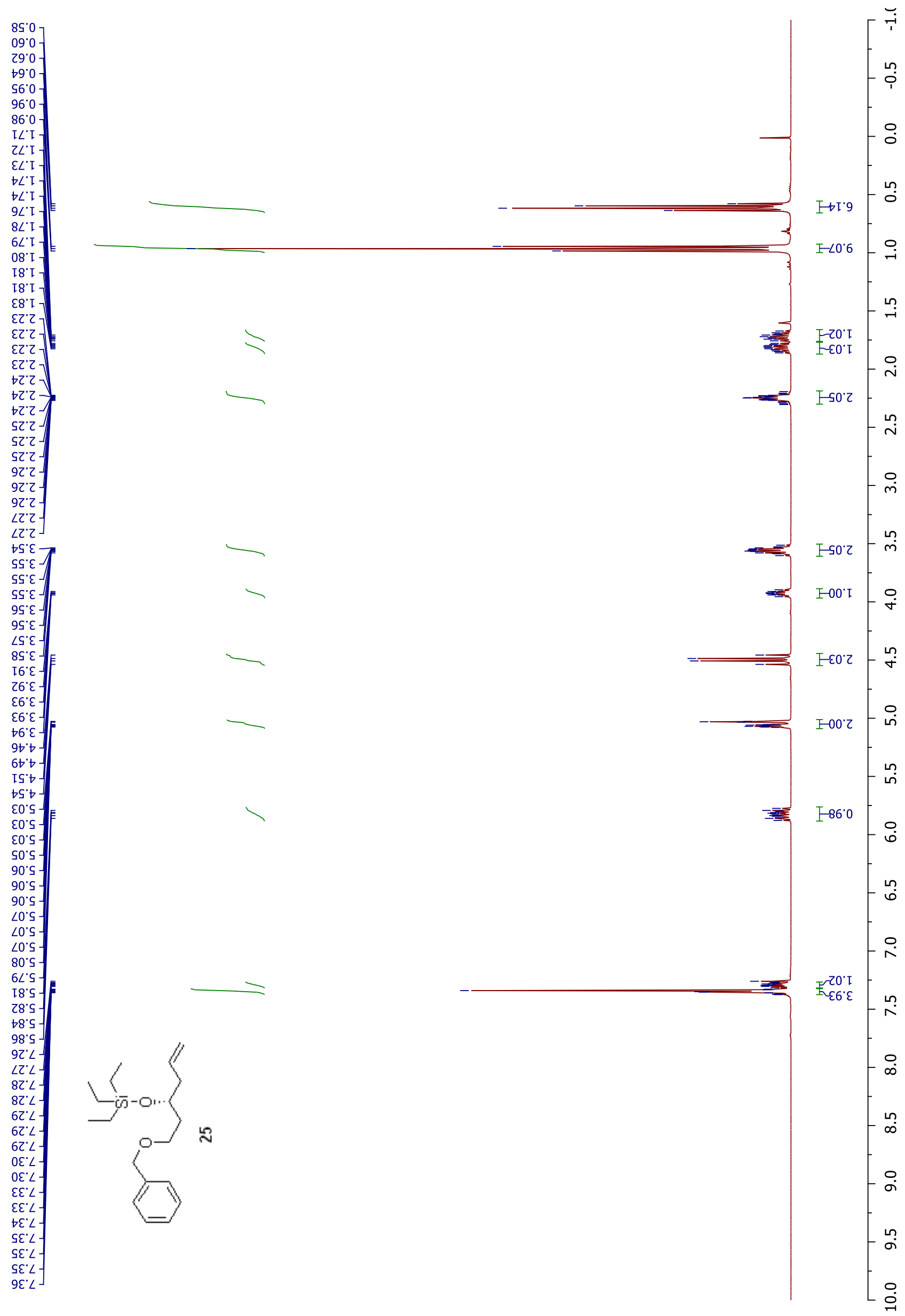


ยั.

$\dashv 8.9 \varepsilon-$

ยเ'てち-

50. $\angle 97$

$00^{\circ} \varepsilon L>$

†० $\angle L$

$9 \varepsilon: \angle L J$

SO $\angle I I-$

IS' $\angle Z[$

$0 \angle \angle Z I \frac{J}{J}$

ธع'8ZI

ย6.

$\angle S^{\prime} 8 \varepsilon I$ -

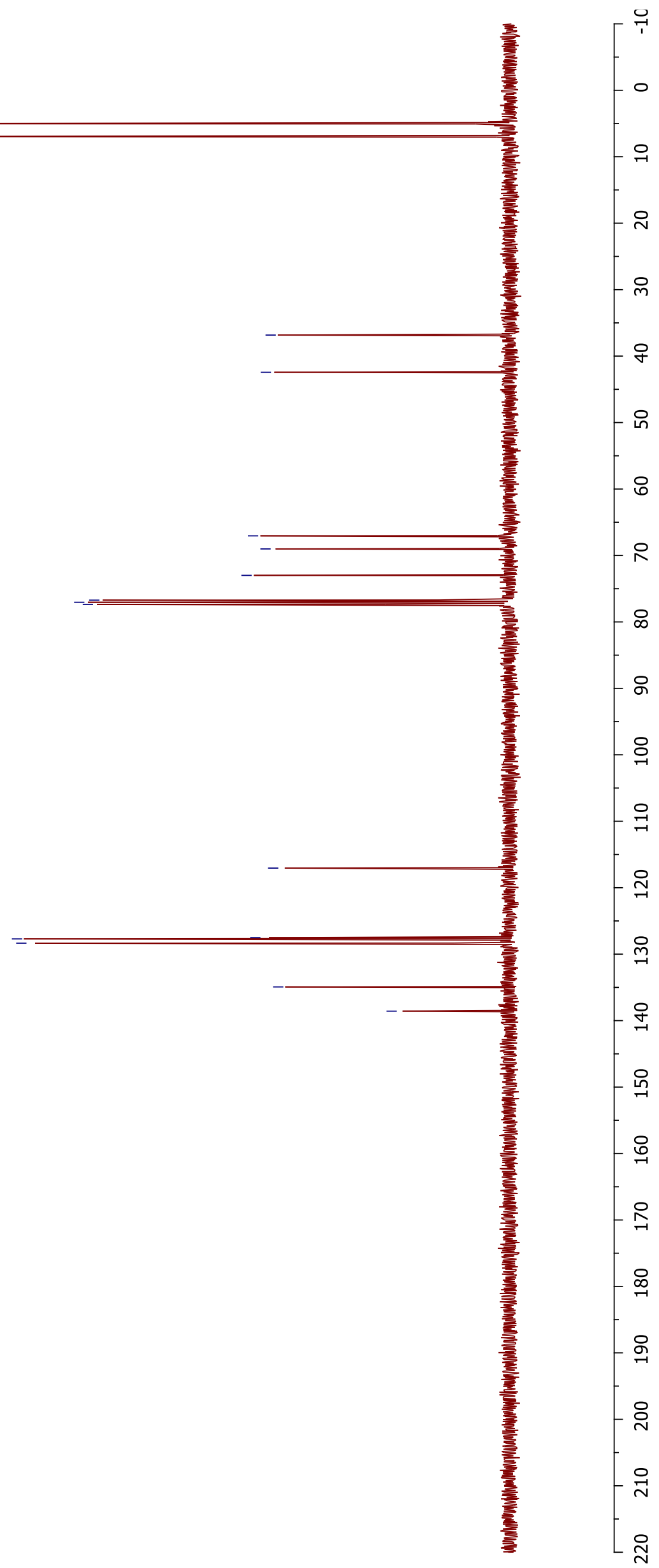




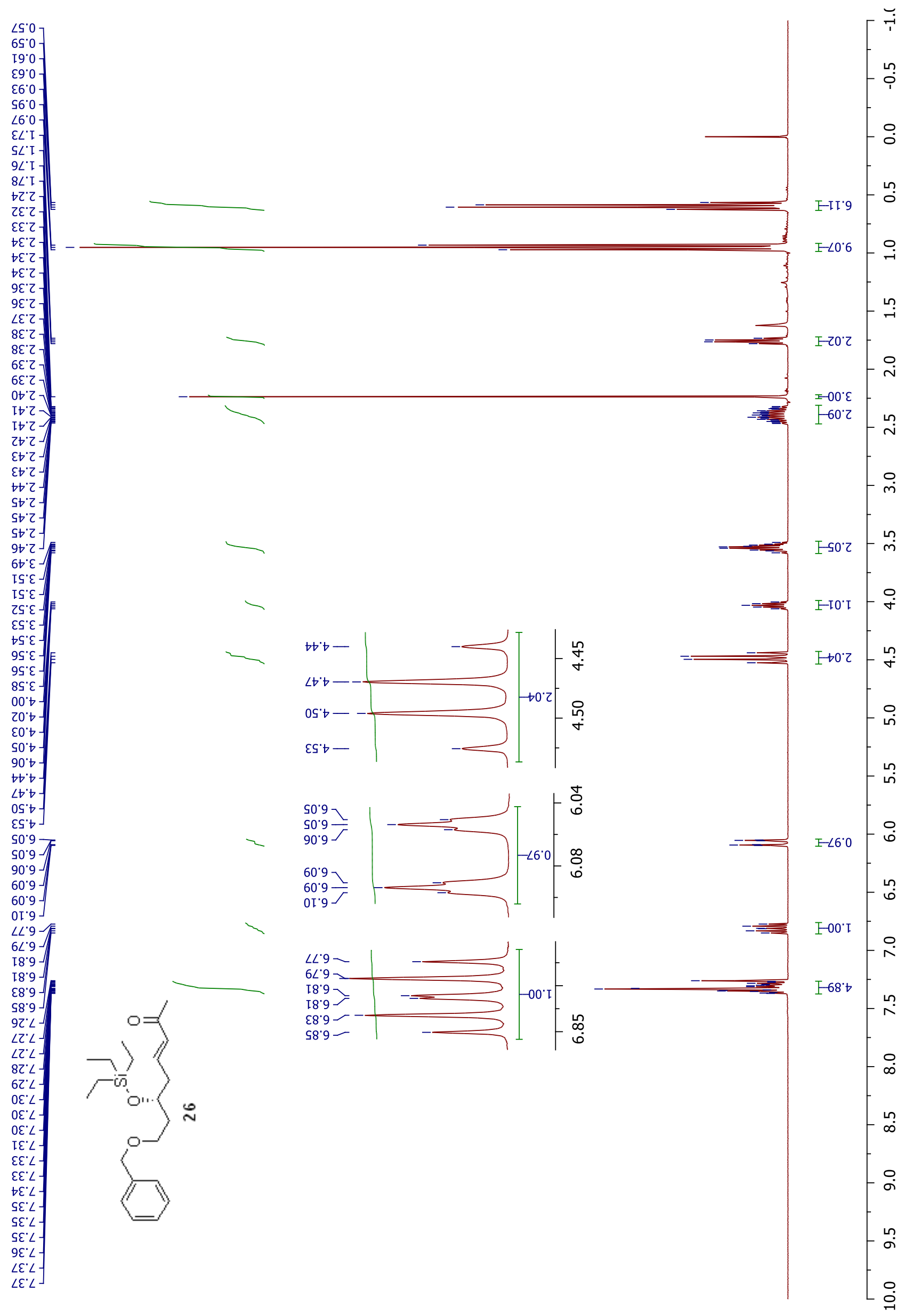


$\angle 6^{\circ} \circ-$

IL'9Z -

$S \mathcal{L} \angle \mathcal{E}-$

$08^{\circ} 0 t$ -

s9.99 乙

七t 89

$60^{\circ} \varepsilon L$

$[\angle ' 9 L]$

$\varepsilon 0^{\circ} \angle L-$

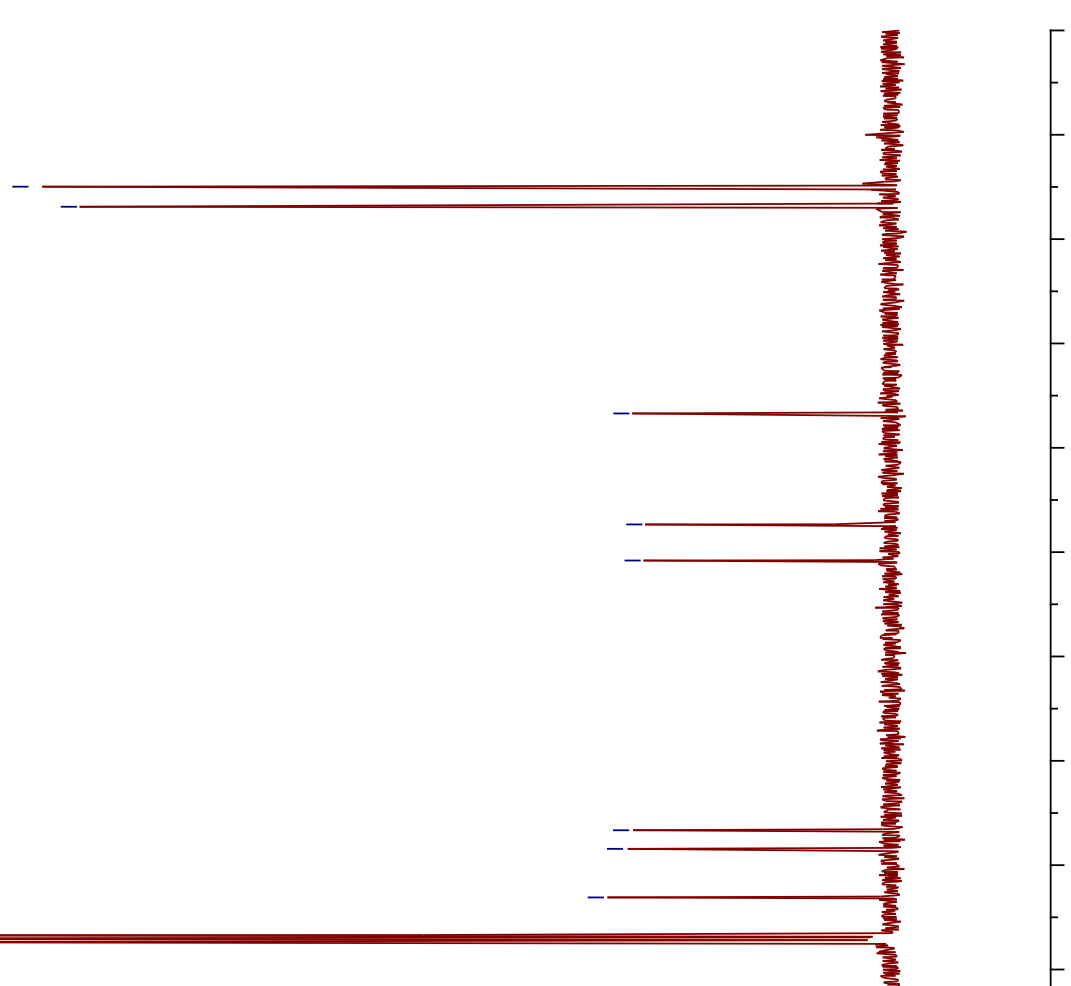

[국

๑ย $8 \varepsilon I$ -

$89^{\circ}$ t十I -
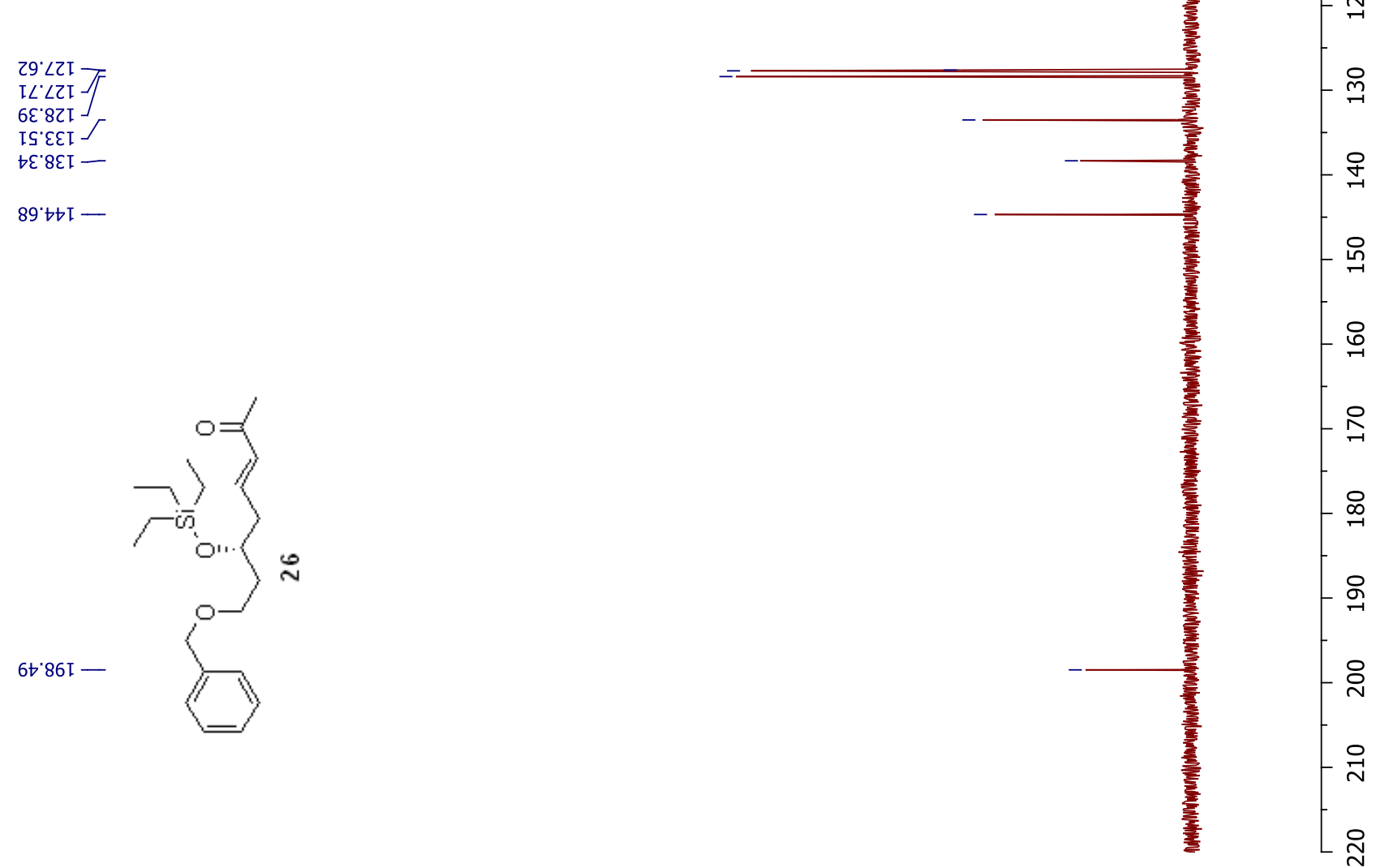


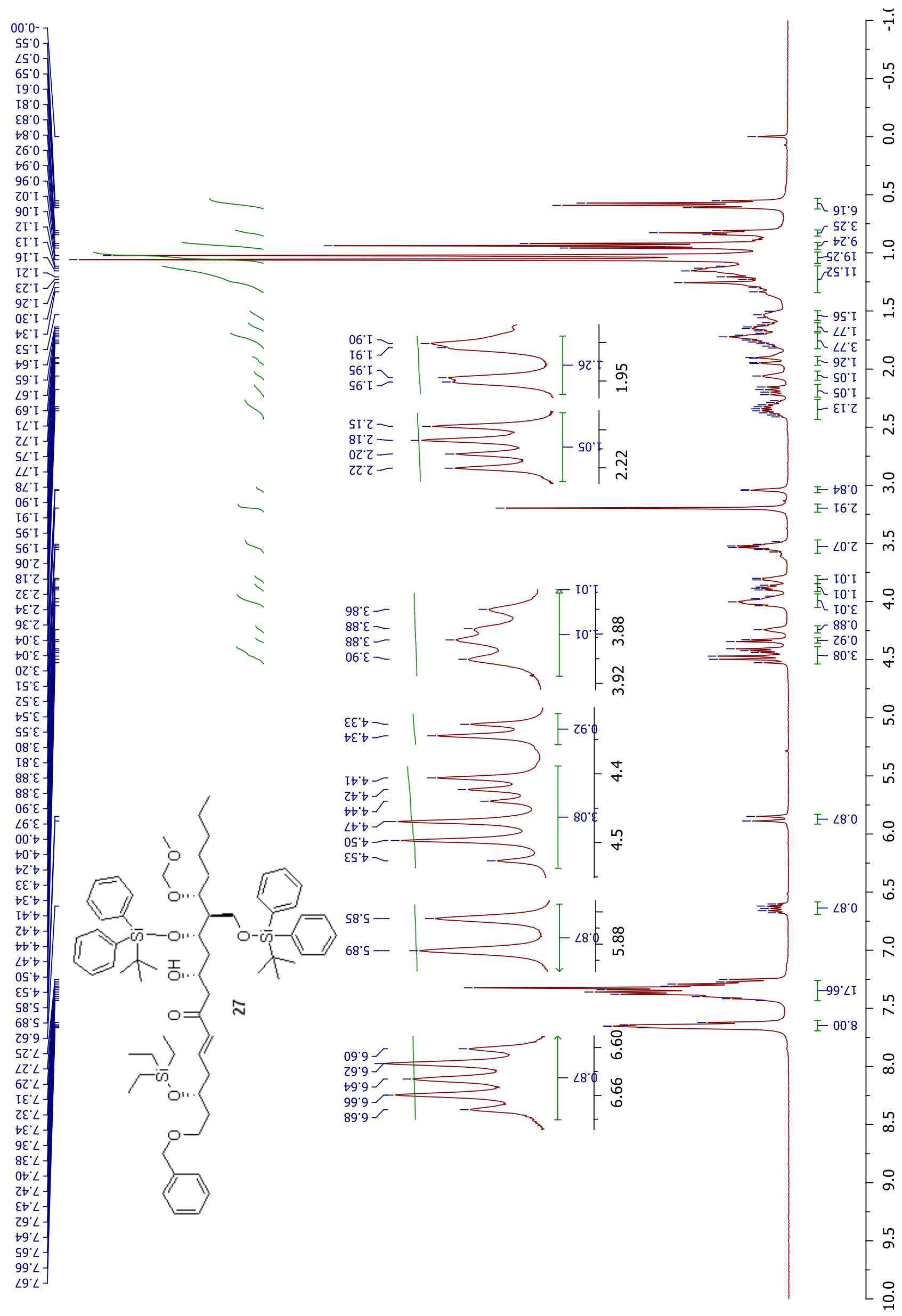



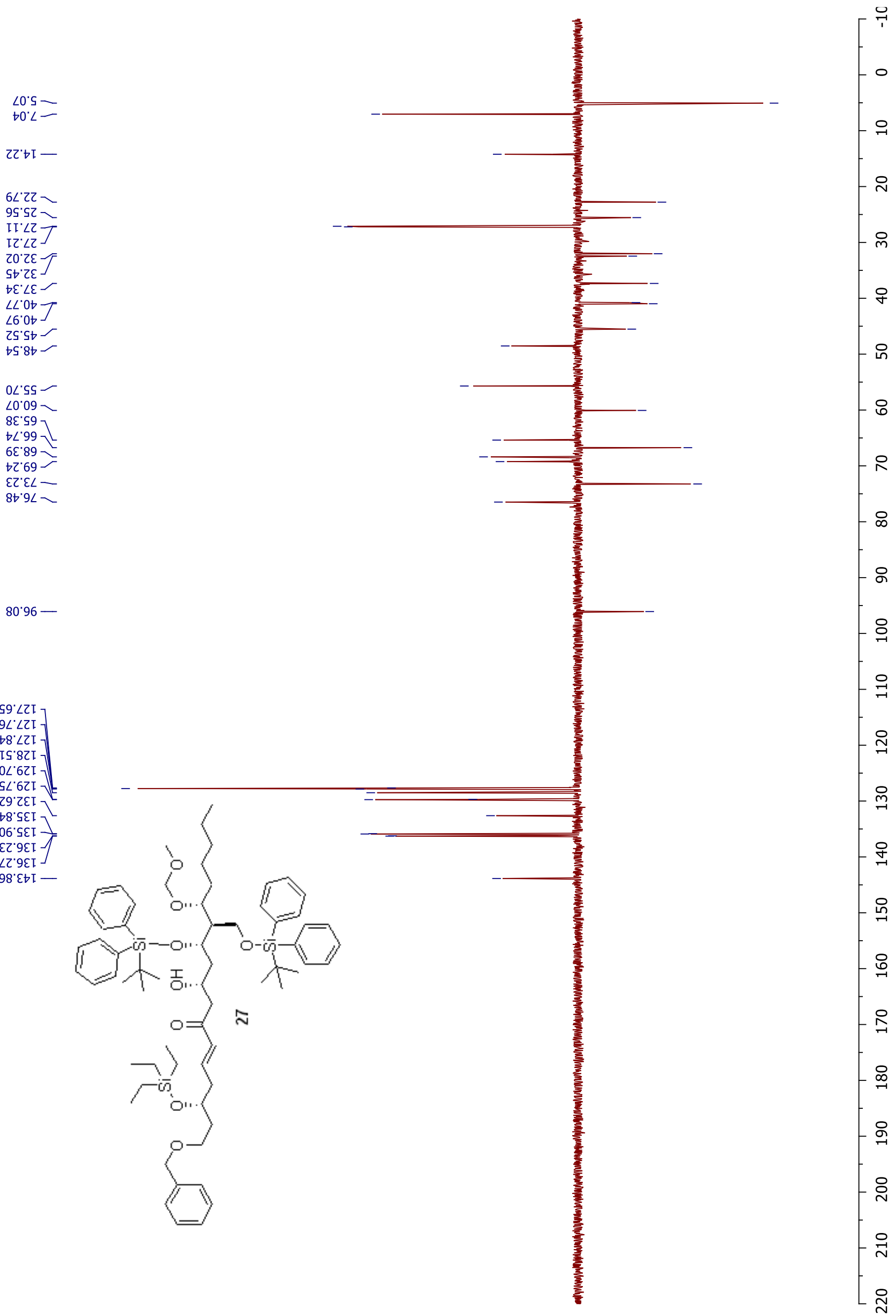

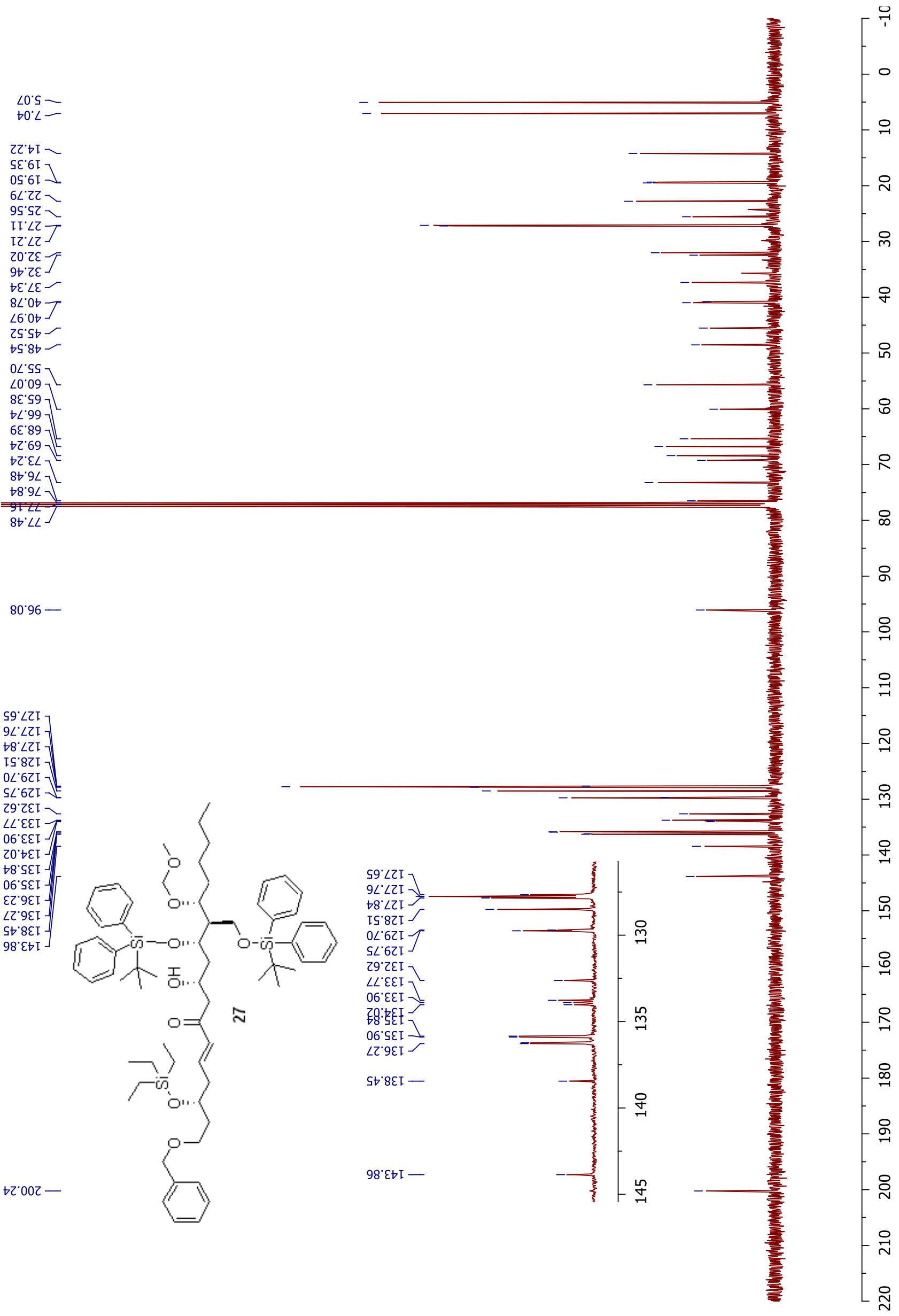


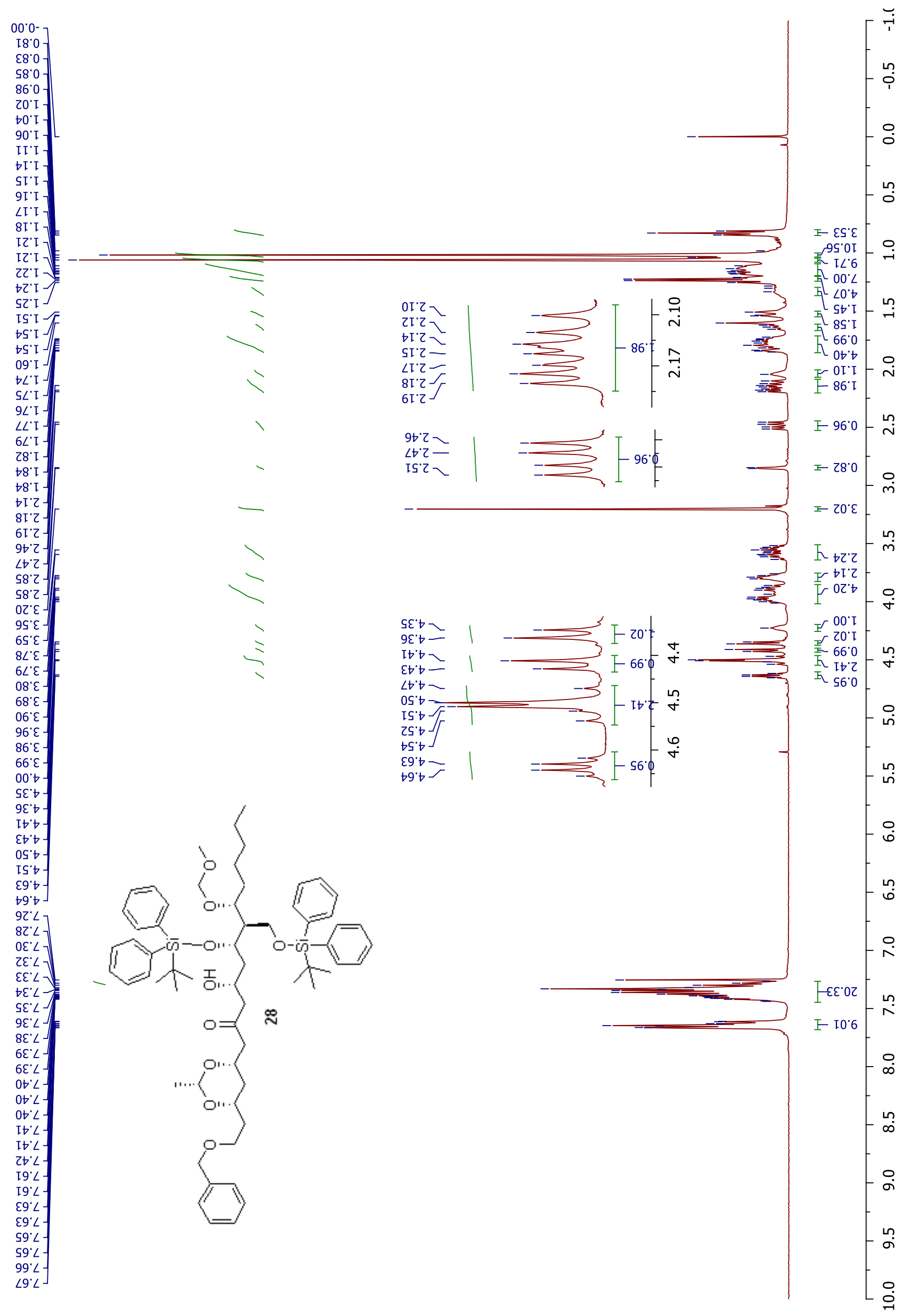


ZZ'เI-

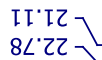

$2+92$ 工

โI'LZ

$8[\cdot[2]$

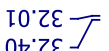

SI'9 $9 \varepsilon-5$

6L'9ع

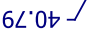

$09.8 \mathrm{~b}$

s0.6t-

$t S \cdot 6 b]$

$\forall \angle$ 'SS -

20.097

$6+59$

0โ' 99

$6 \varepsilon 69$

OI' $2 \angle$

OT' $\varepsilon L$

$8 \mathrm{~T} \cdot \varepsilon L>$

ZS' $9 L-$

tน.96-

$\angle 9.86$ -

$89^{\circ} \angle Z I$

$\forall \angle \angle Z I]$

$8 L^{\circ} \angle Z T$

IS'8ZT

$80^{\circ} 62 \mathrm{I}$ -

†8 ऽ\&I

88. जEI J

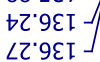
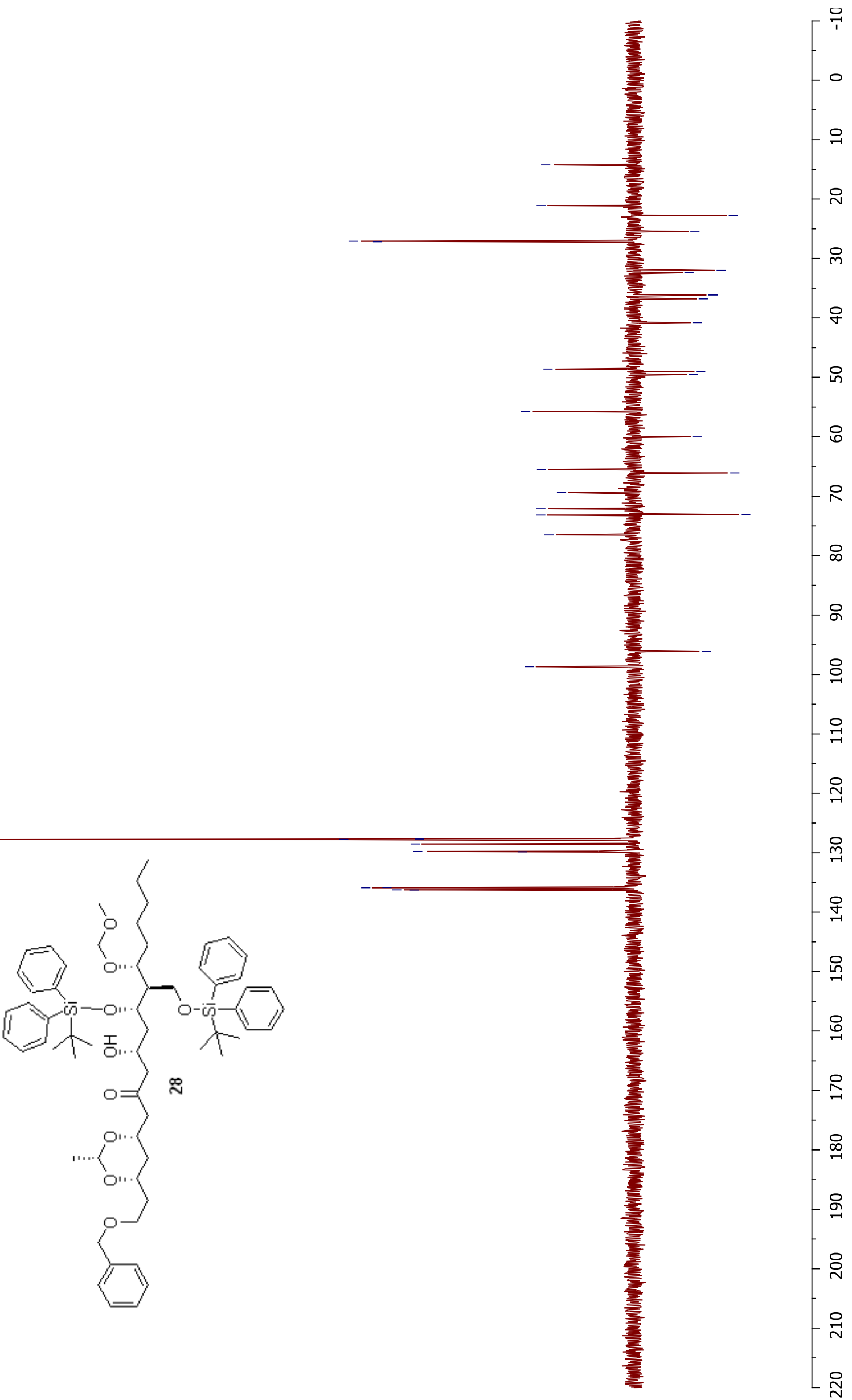


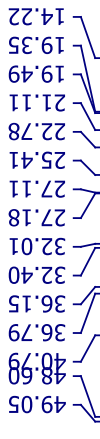

tLSS

20.09

66.59

0['99]

$05^{\circ} 69$

Oโ' $Z L$

$0 I^{\prime} \varepsilon L$

$8 I^{\prime} \varepsilon L$

ح'. $9 L$

$9 I^{\circ} \angle L-$

$8 b^{\circ} \angle L$

†I' 96

$\angle 986$

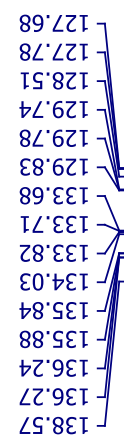

$80^{\circ} 602-$

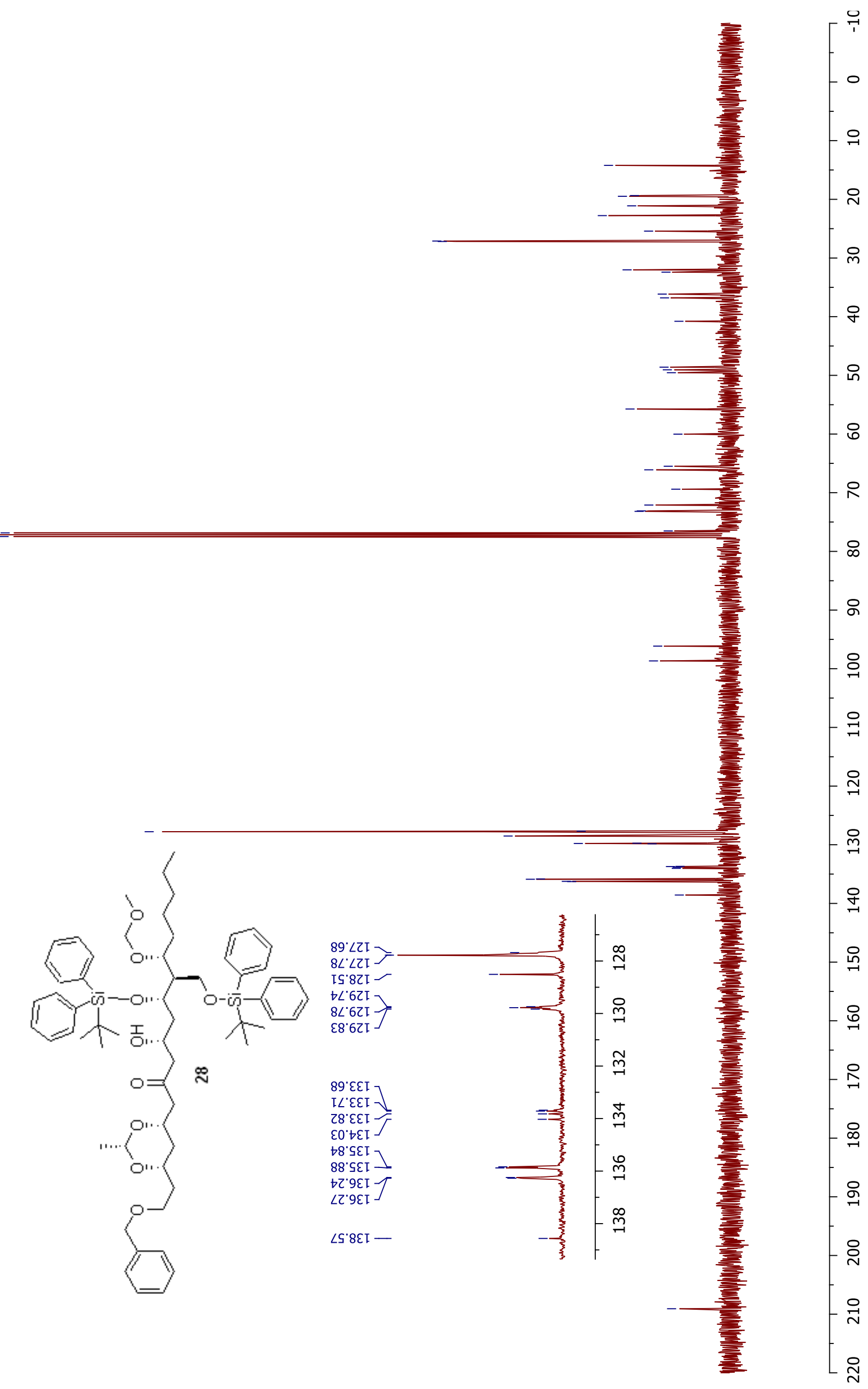




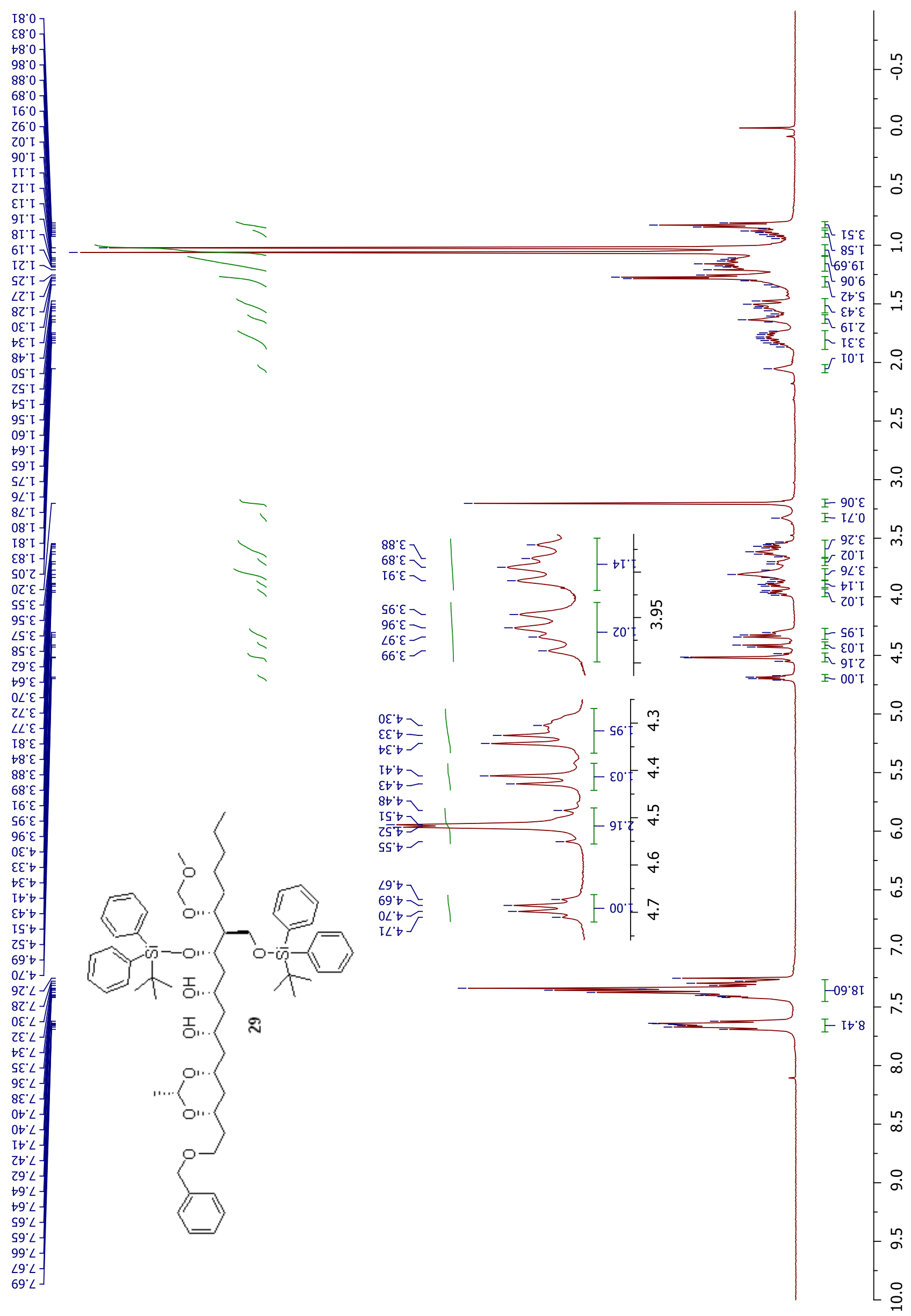




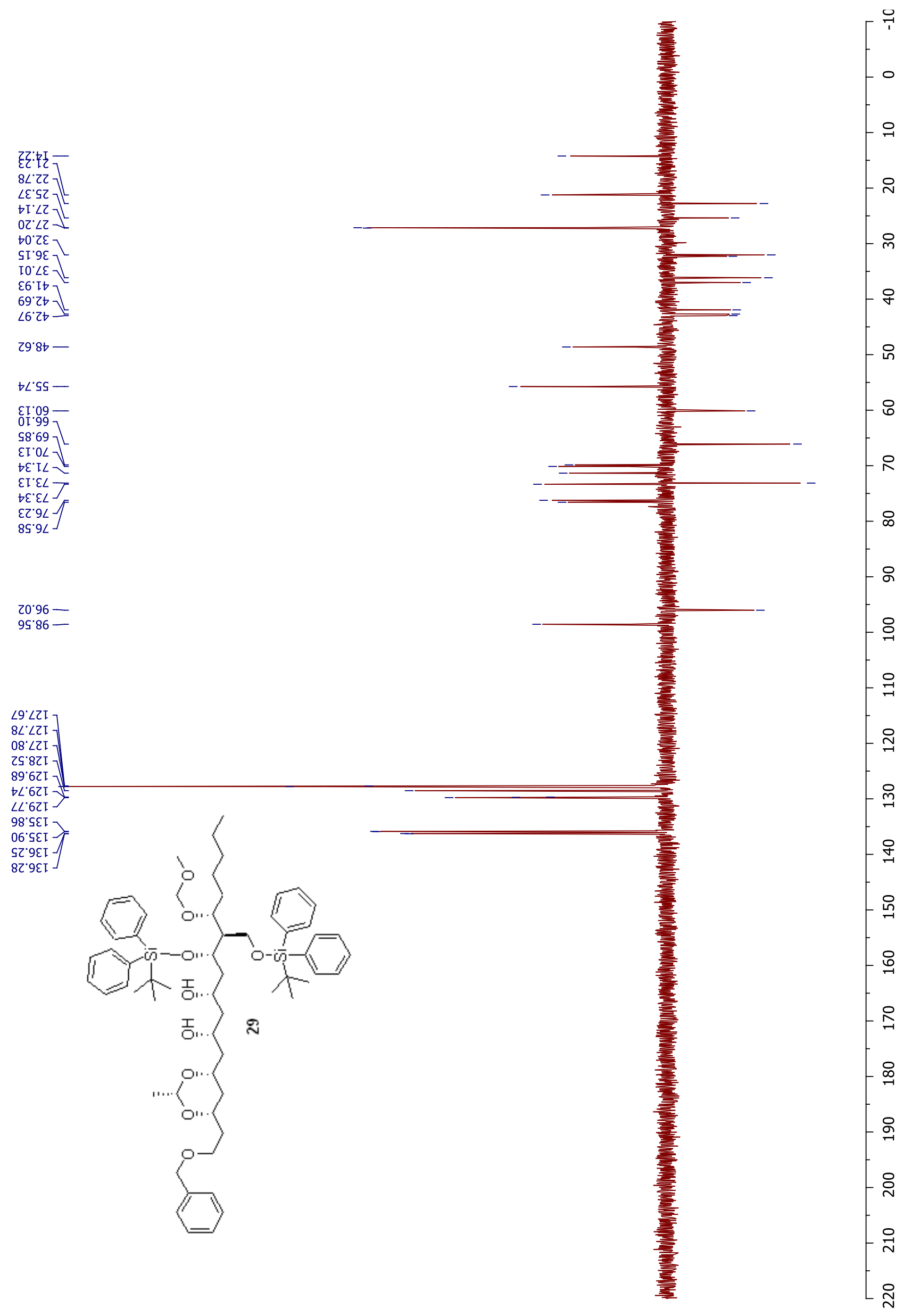




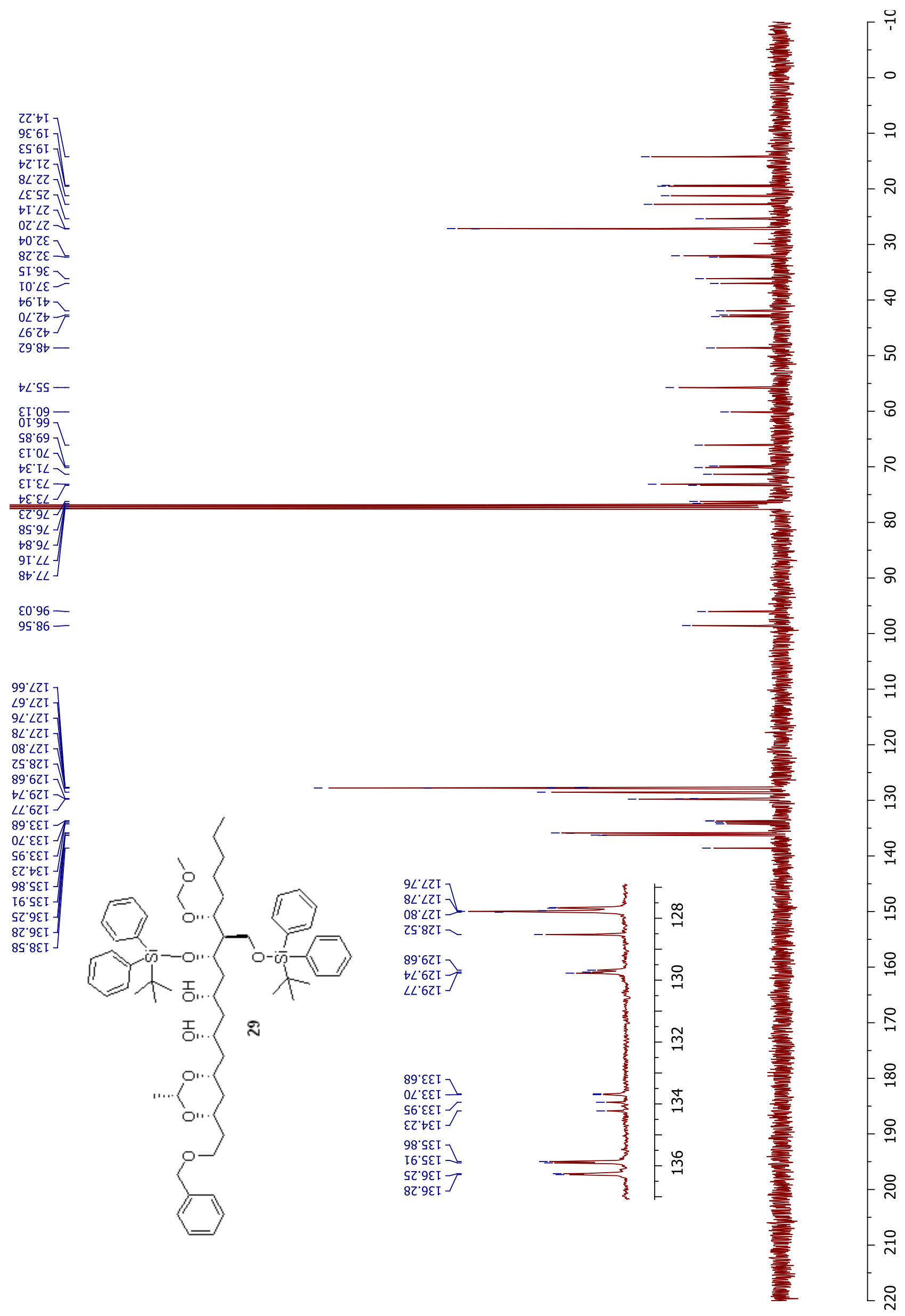



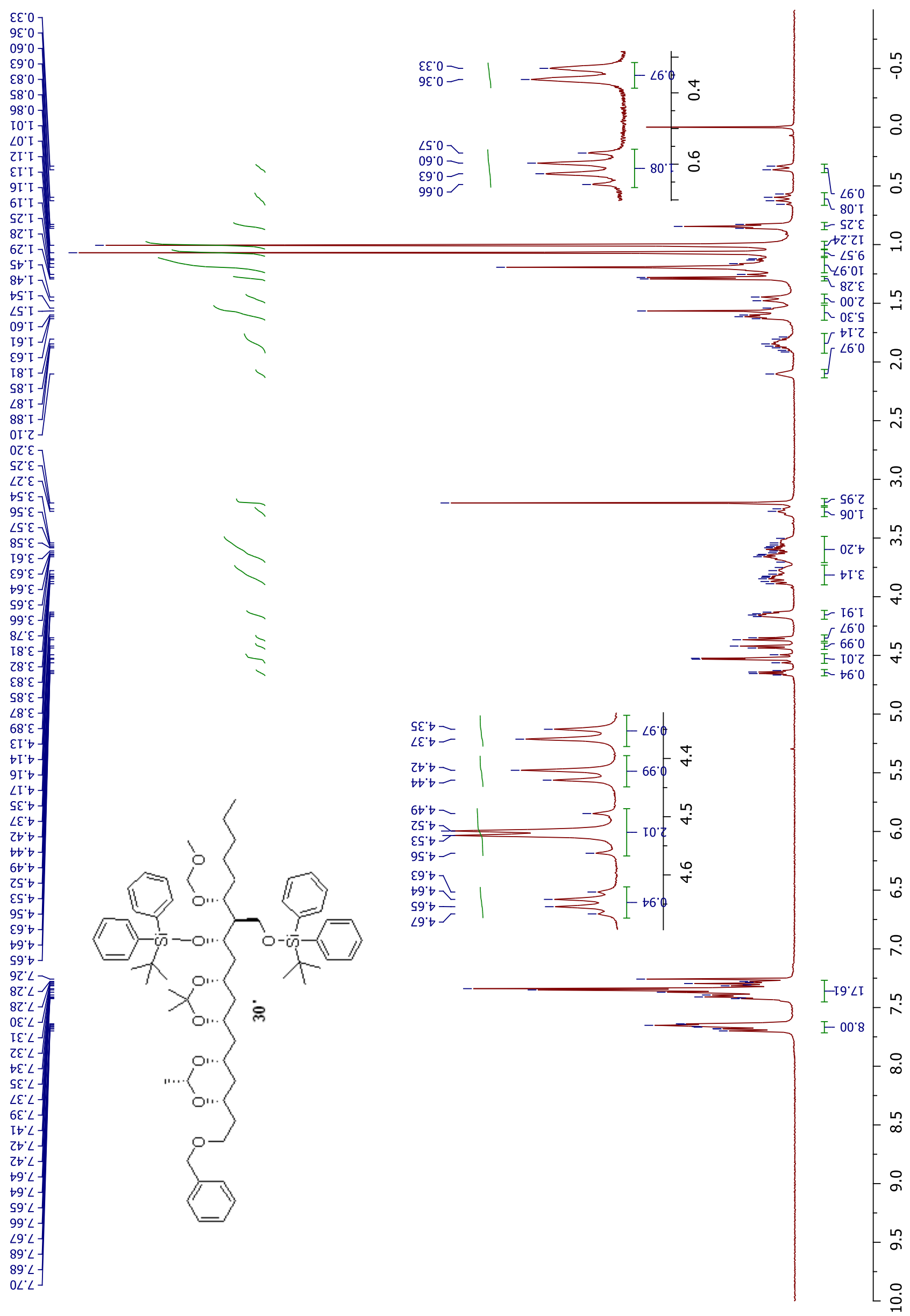

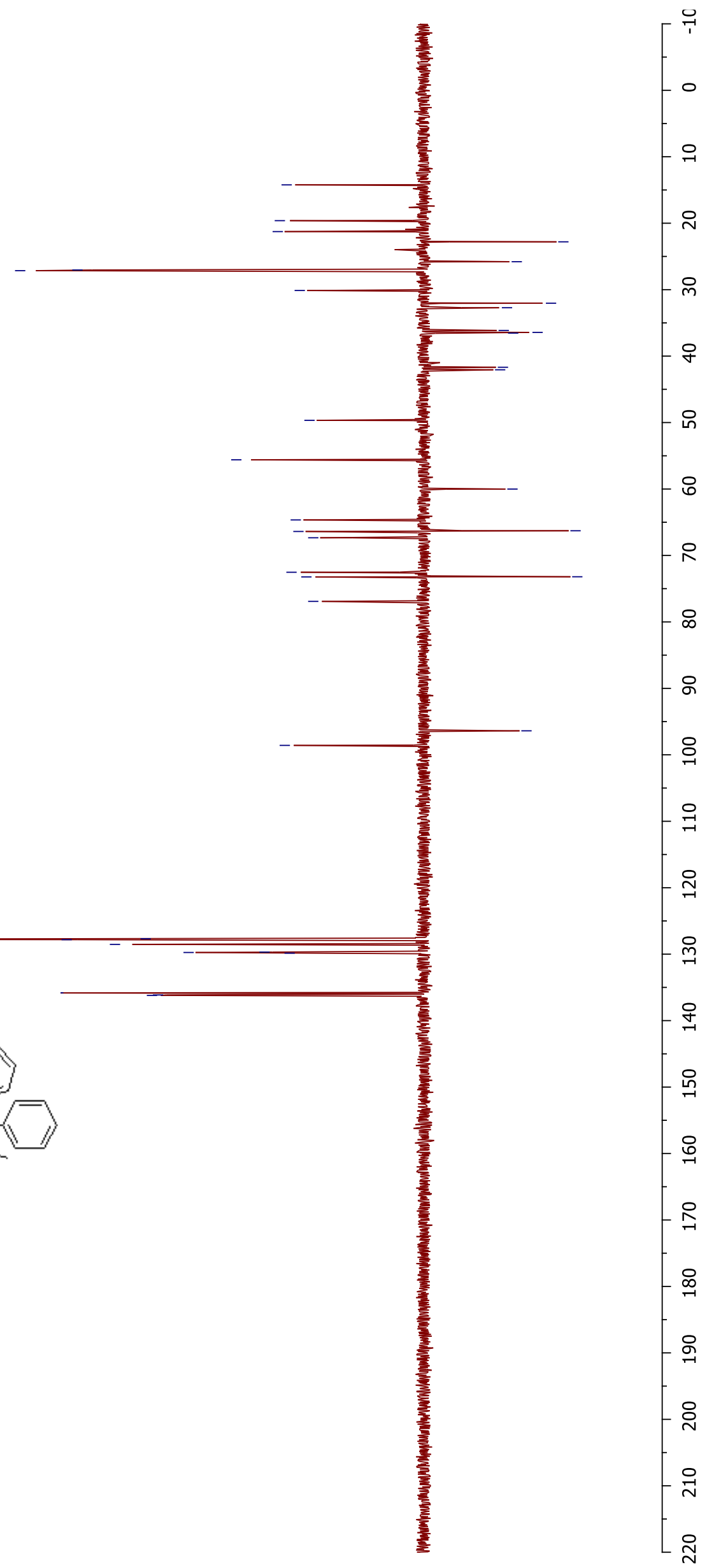


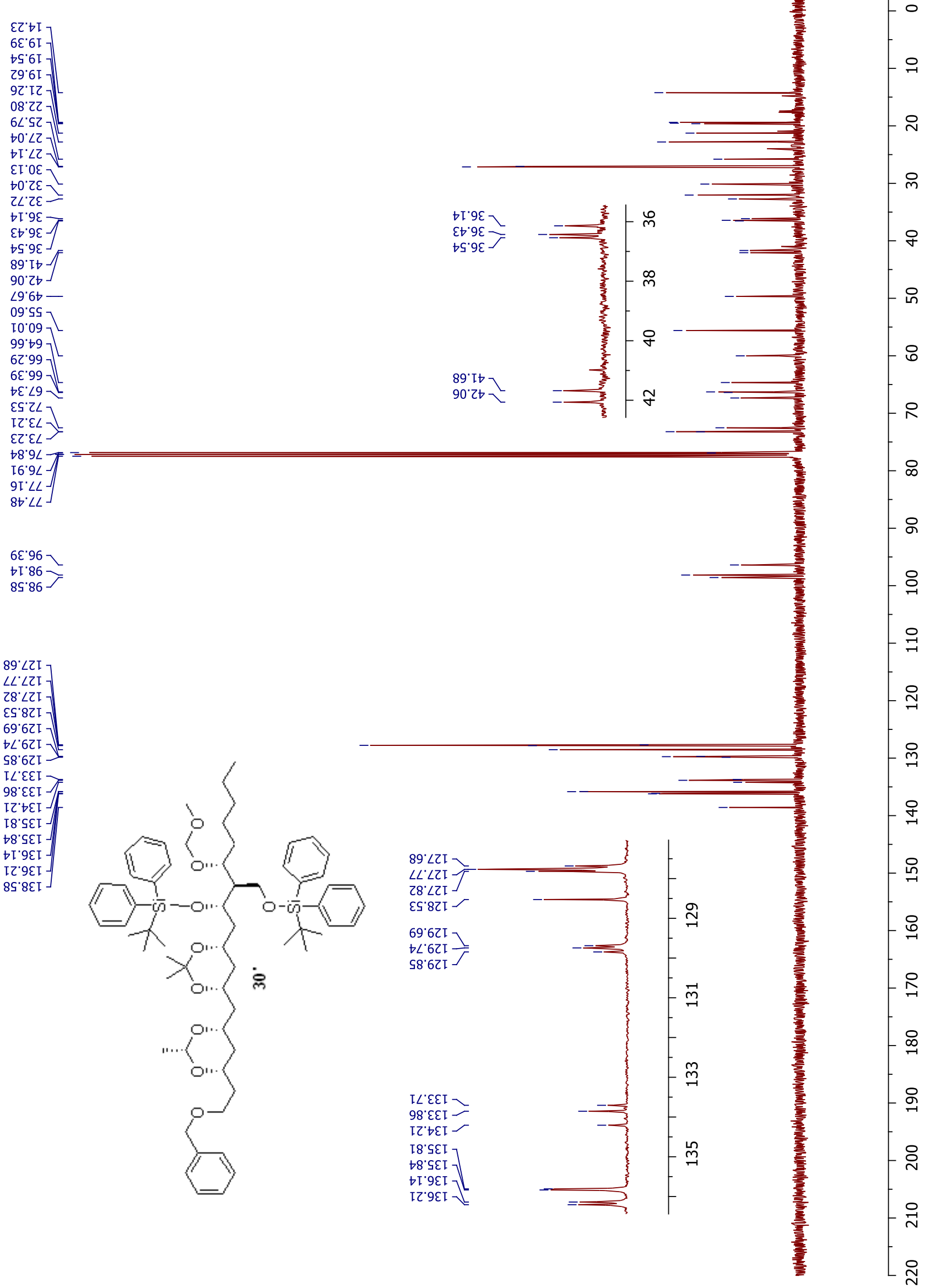



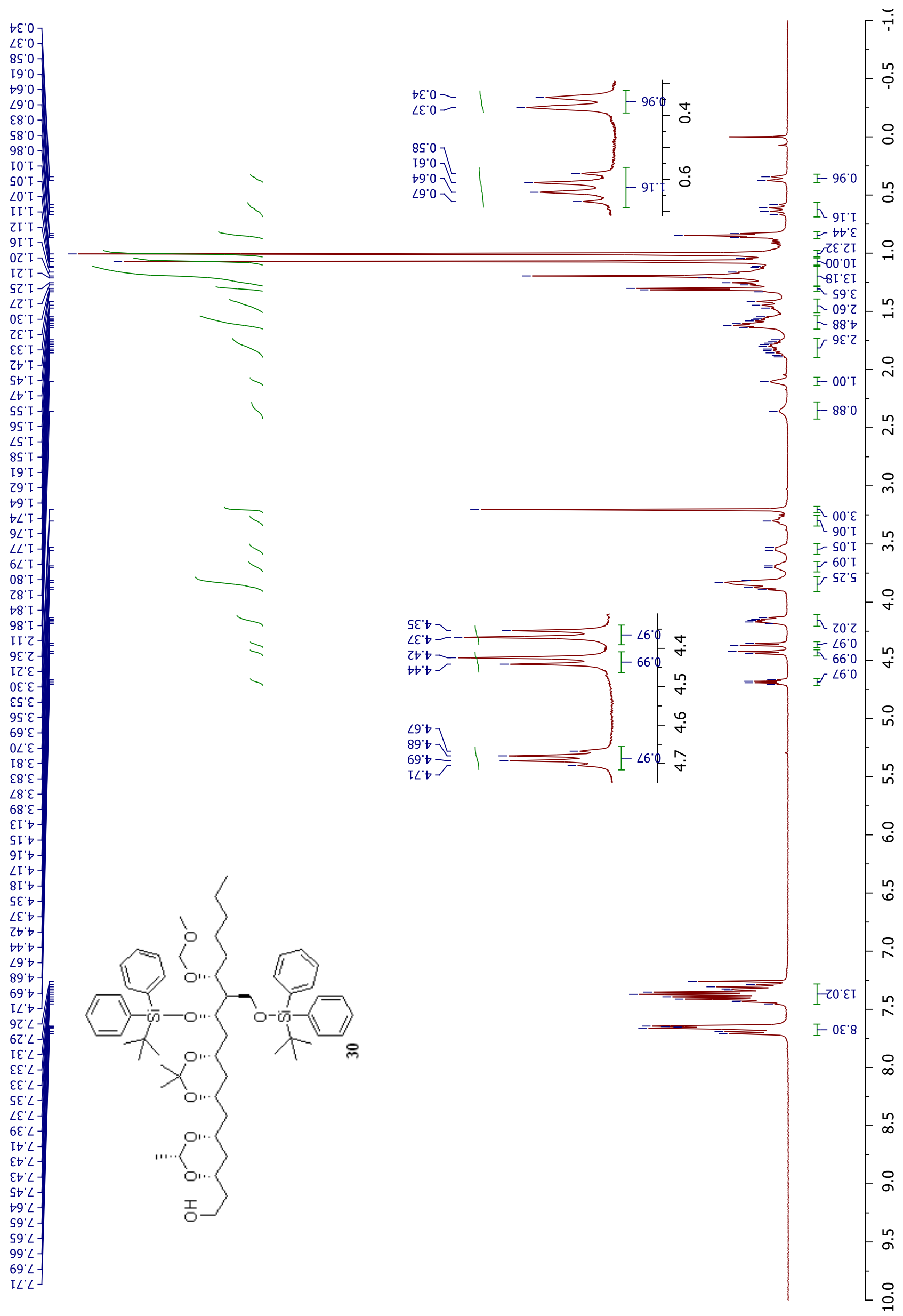
$\left.\begin{array}{l}\varepsilon Z^{\circ} \triangleright[ \\ \varepsilon 9^{\circ} 6[\end{array}\right]$

โย' $น 乙]$

$\left.08^{\circ} \mathrm{ZZ}\right]$

$08.52]$

$\rightarrow I \cdot L C \sqrt{2}$

$\varepsilon I^{\circ} 0 \varepsilon$

So'ZE ไ

$\varepsilon L ' \tau \varepsilon$

0โ' $9 \varepsilon>$

I乙'9ع

$60{ }^{\circ} 8 \varepsilon$

99. It

26. It

99.6b-

I9'SS -

10.09

28.09 -

$89^{\circ}+9$

$0 t^{\prime} 99-$

$9 \varepsilon^{\prime} \angle 9 \Omega$

$\angle S ' Z L$

S6.SL

โ6.9L

$05^{\circ} 96-$

โ9.86-

69. $\angle 2 I$

$\angle L ' \angle Z I]$

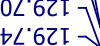

98.62I

โ8'SEI

๑8. SEI

$\rightarrow$ † $9 \varepsilon[-$

[Z'9EI
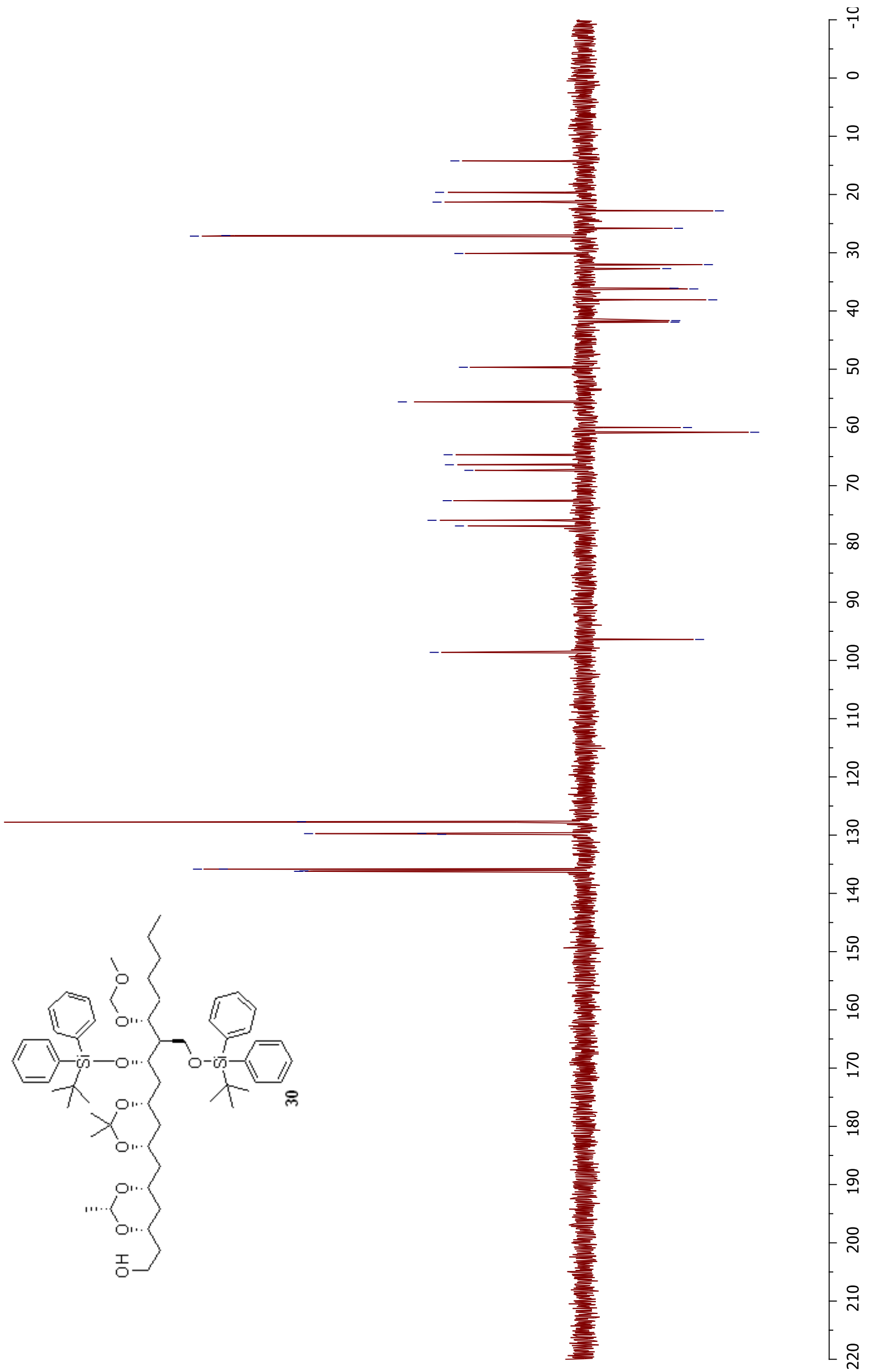


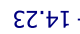

$0 t^{\circ} 6 \mathrm{I}$

SS.6I ]

โย'น乙]

โ8.22]

$\left.08^{\circ} \mathrm{s}\right]$

50:22

SI' $\angle Z \sqrt{ }$

SO'ZE

$\varepsilon / 2 \varepsilon>$

$0{ }^{\prime} 9 \varepsilon$

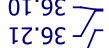

$60.8 \varepsilon$

$\angle 9^{\circ}$ It

26. It

99.6t-

29.5

[0.09

ع8.09

$69^{\circ}+9$

[t.99 ]

$\angle \varepsilon^{\circ} \angle 9$

8S' $2 L$

S6 $S L]$

$78^{\circ} 9 L$

$8 b^{\circ} L L$

$0 t^{\circ} 96$ 工

SI. 86

29.86

$0 L^{\circ} \angle Z I$

$\angle L^{\prime} \angle Z I$

$\checkmark L \cdot 62 I]$

98.62I V

69. દย

98.

ธน' $\downarrow \varepsilon I-\bar{F}$

Z8. ऽEI -

† ' '

Sโ'9ع ]
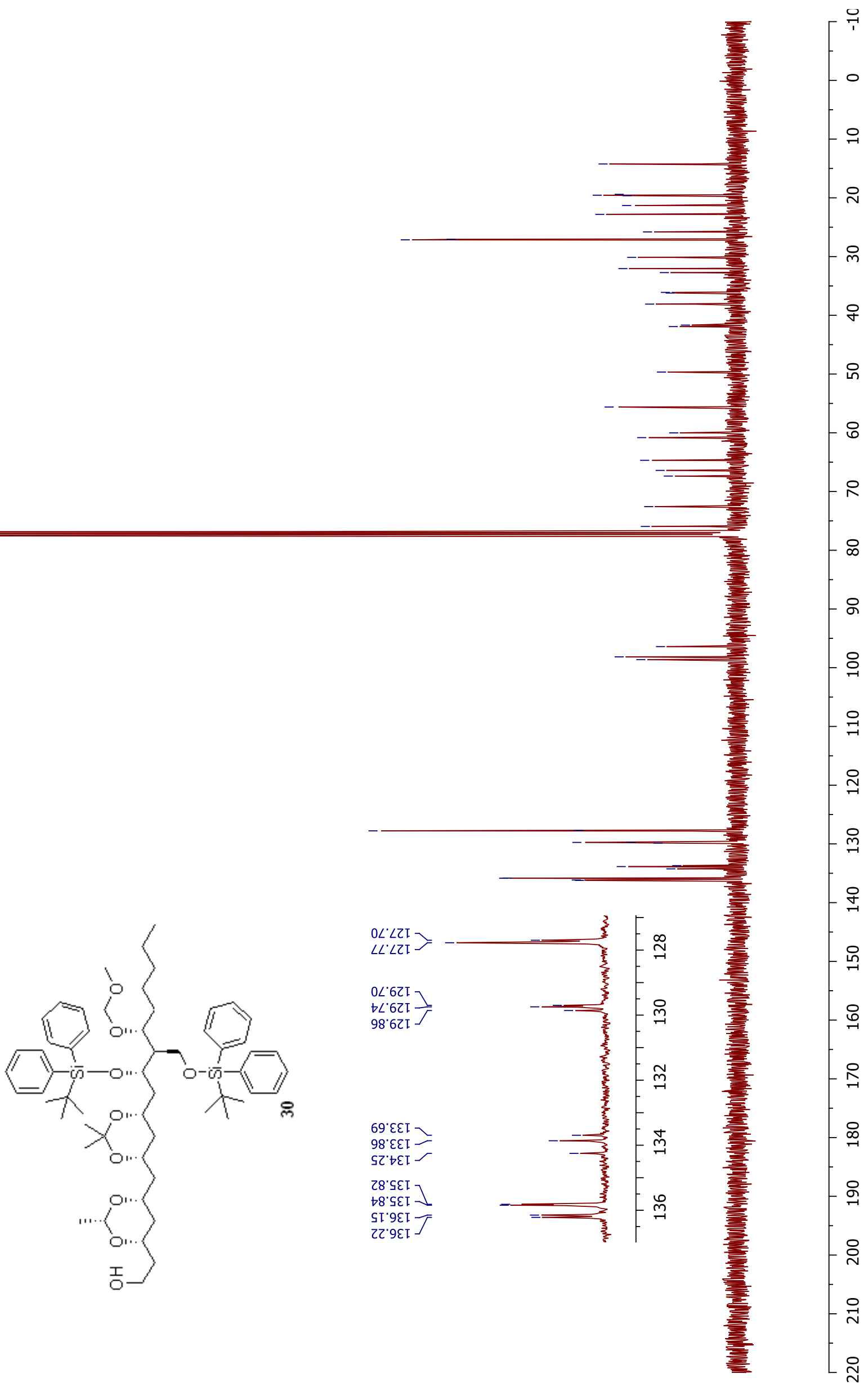

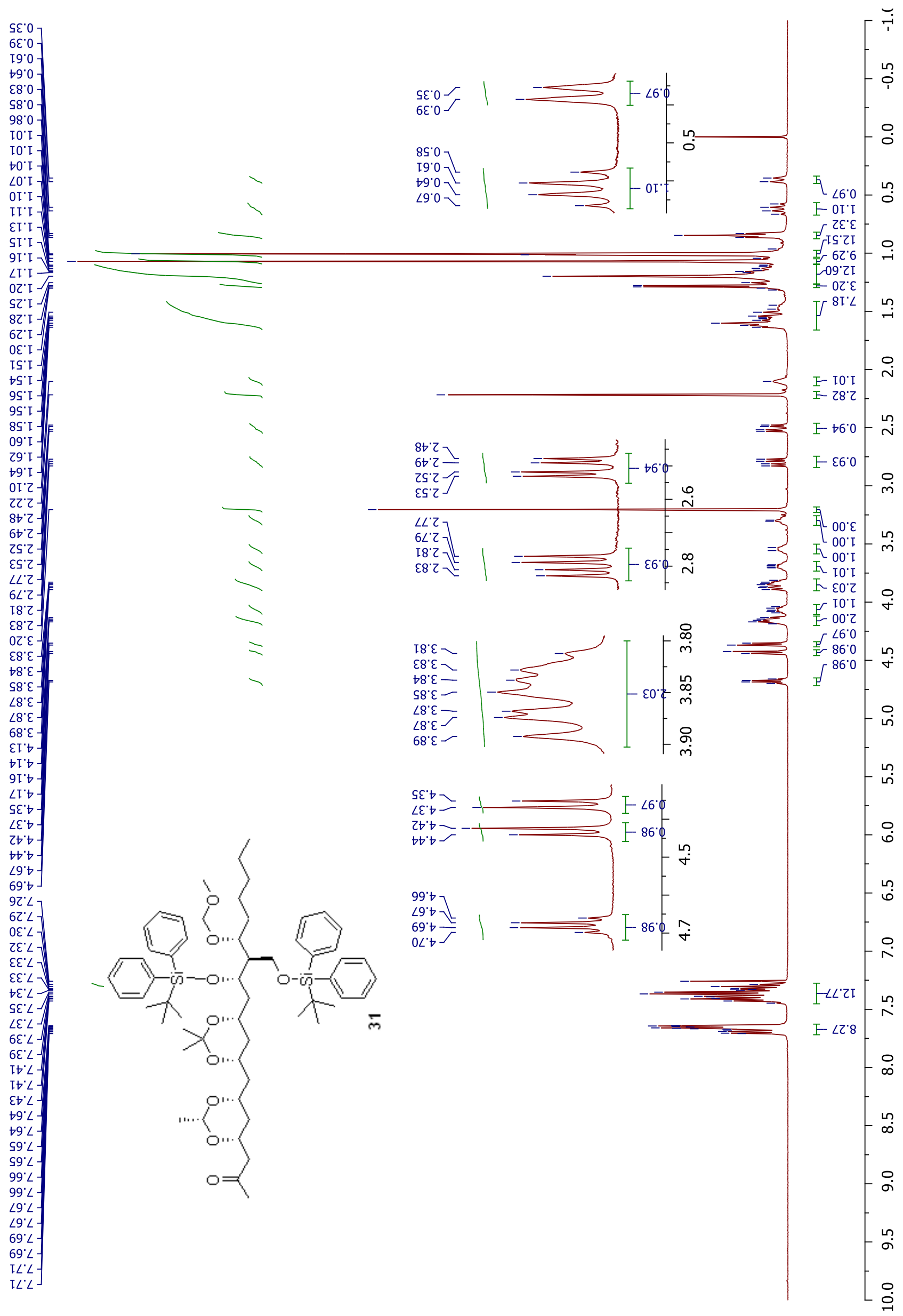


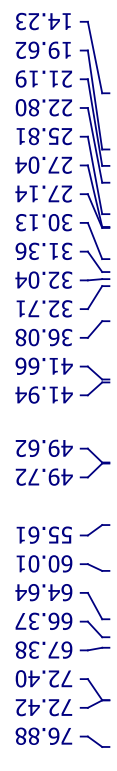

$\angle \varepsilon \cdot 96-$

$\angle 9^{\circ} 86$ -

$89^{\circ} \angle Z I_{7}$

$\angle L ' \angle Z I]$

$\rightarrow\left[{ }^{\circ} 62 \mathrm{~L}\right]$

ह8.62I

โ8. SEI

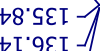

$\neg$ โ $9 \varepsilon[-$
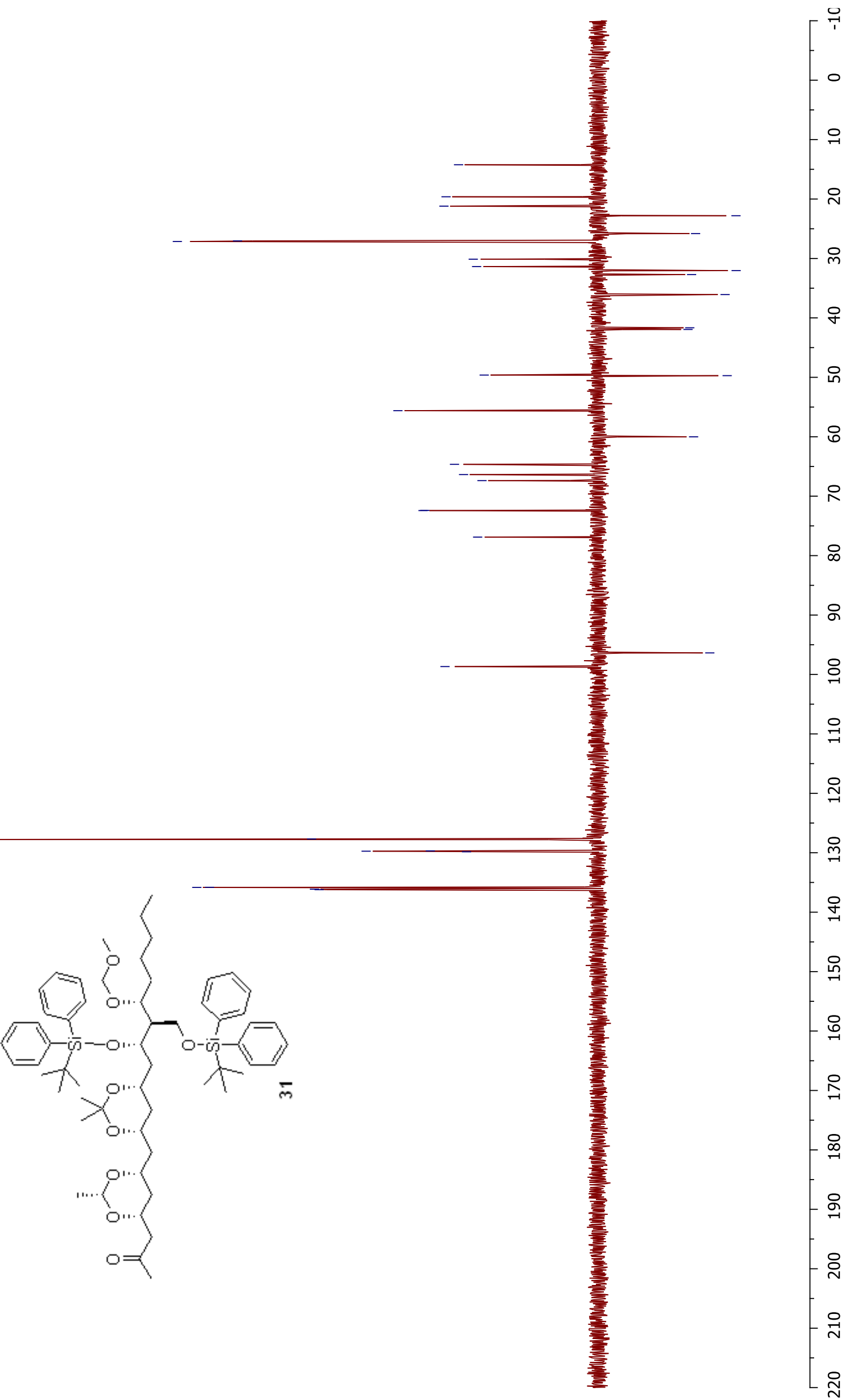


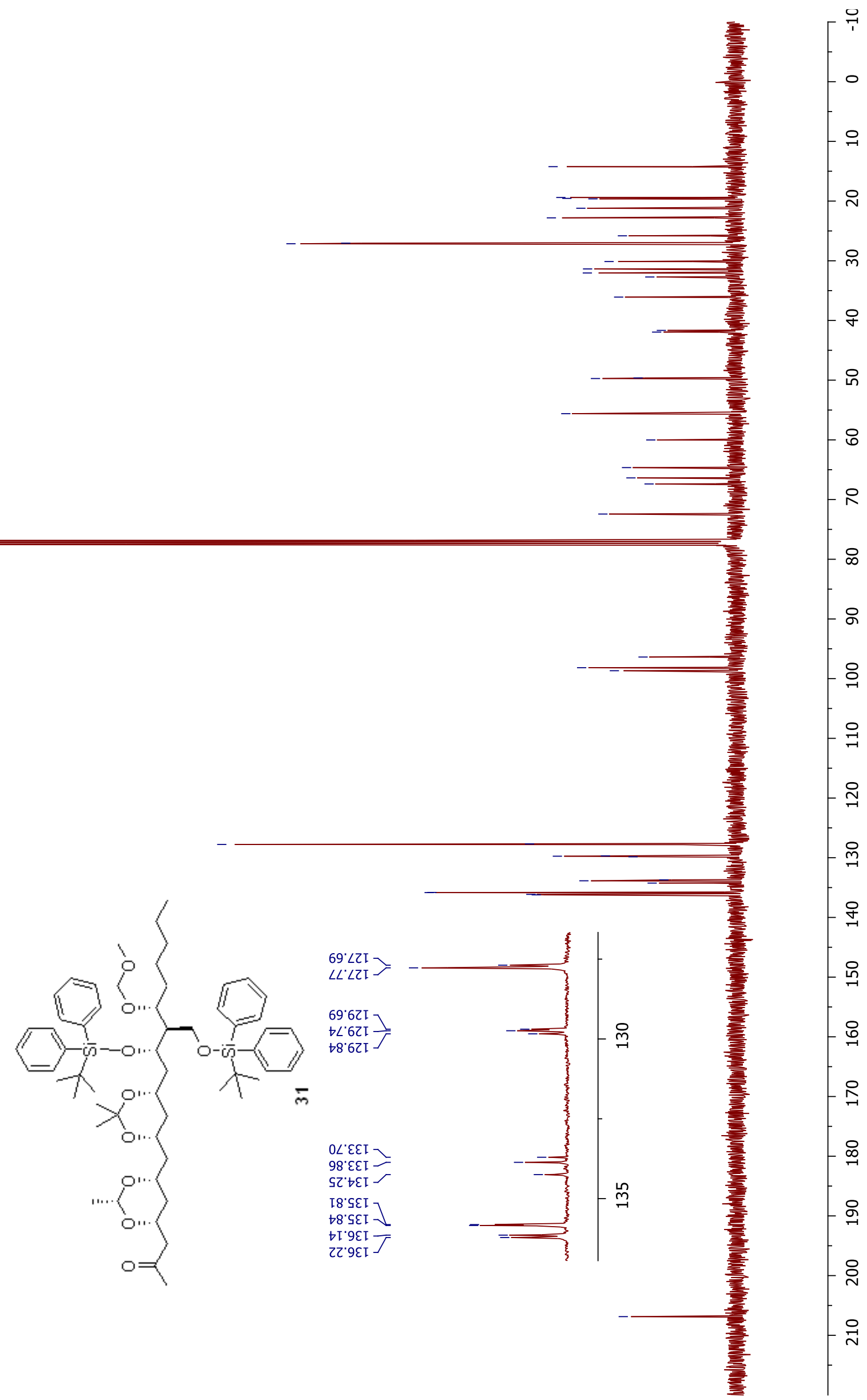




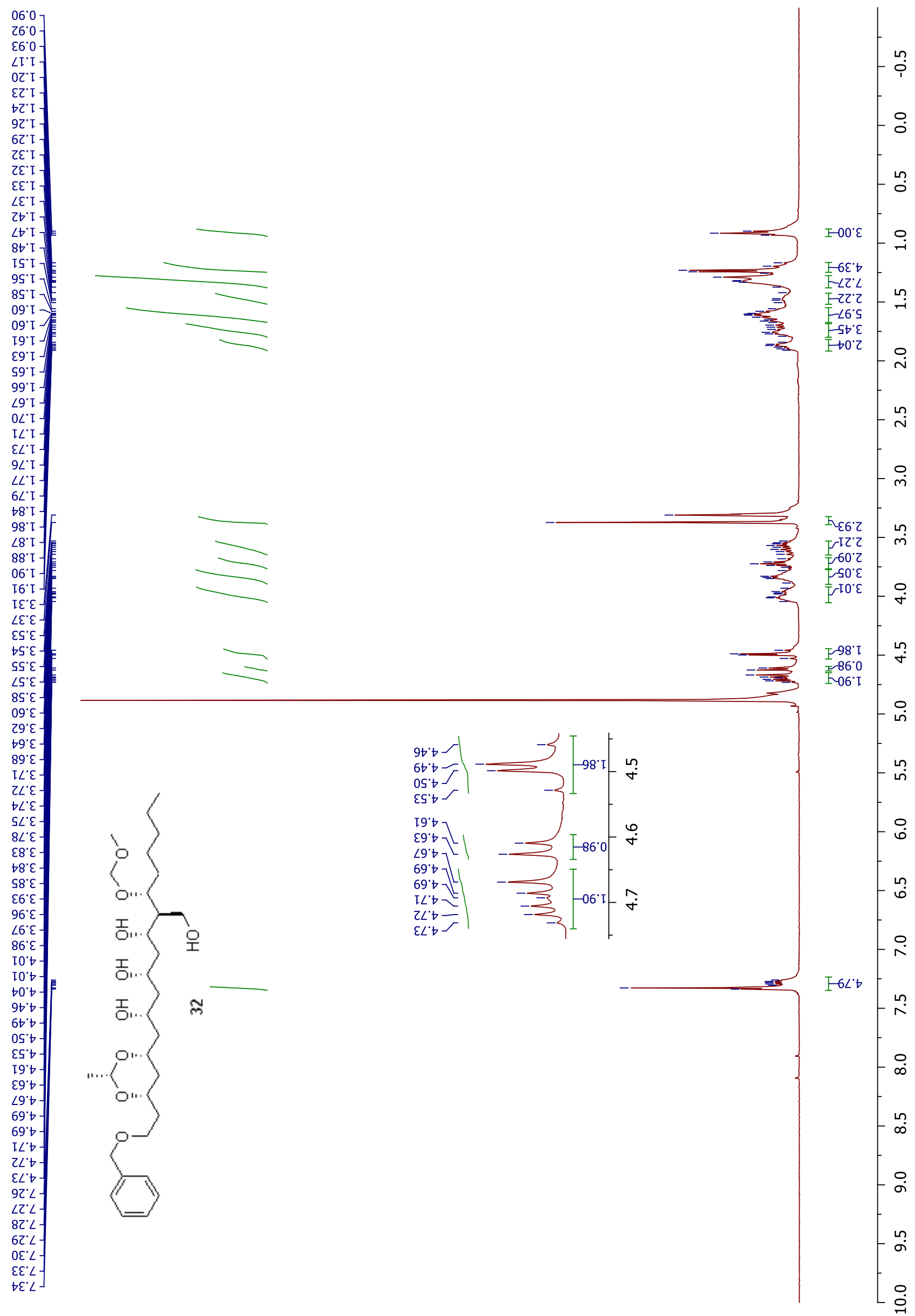




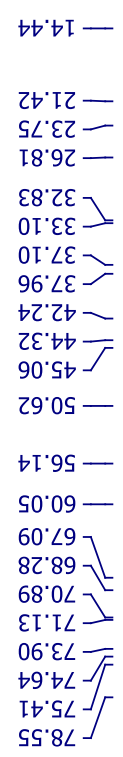

$8 \varepsilon \angle 6-$ ZL' 66 -

$\angle 9^{\circ} 8 Z I$
$\left.\angle 8^{\circ} 8 Z I\right]$
$\angle E^{\circ} 6 Z I$
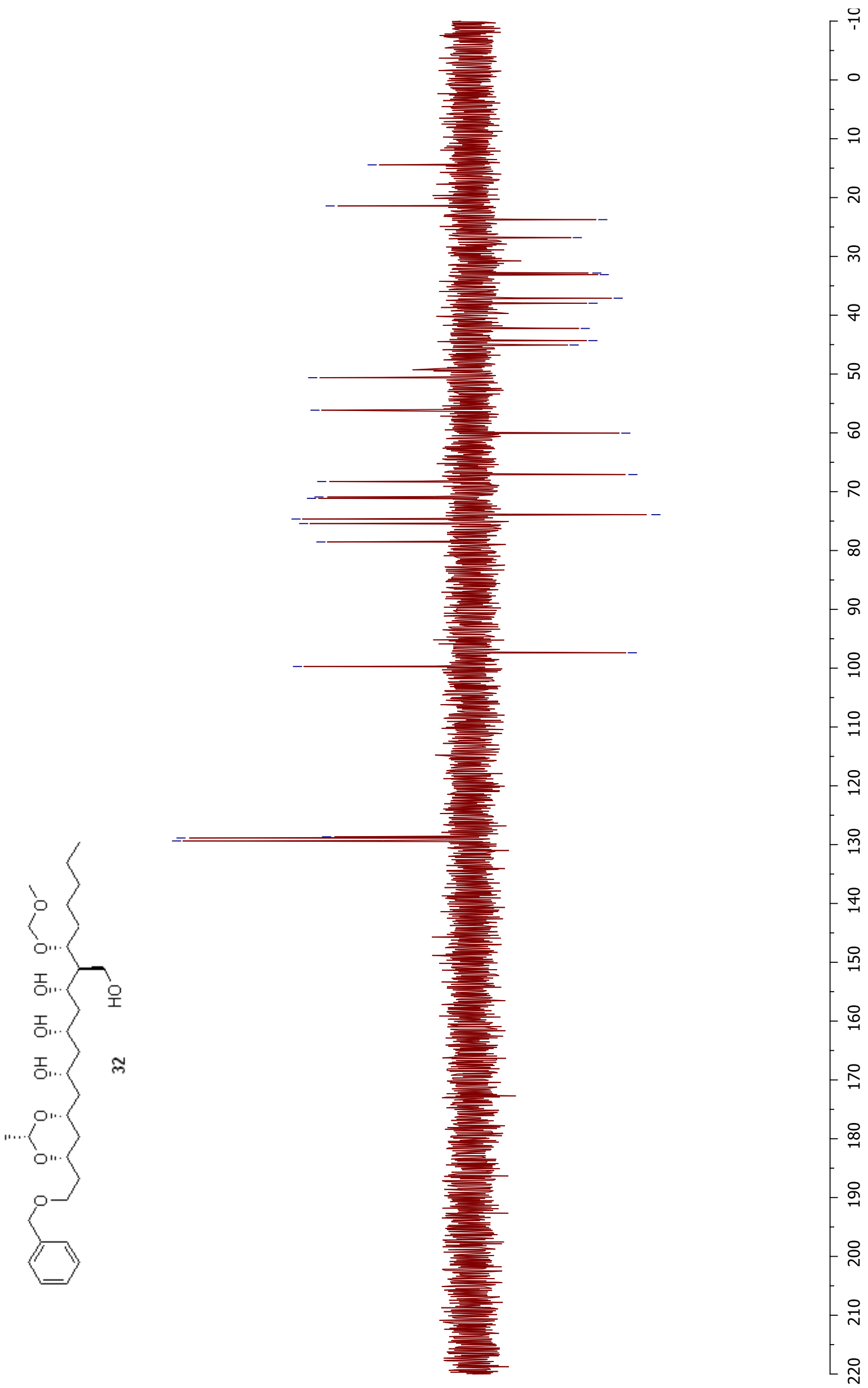


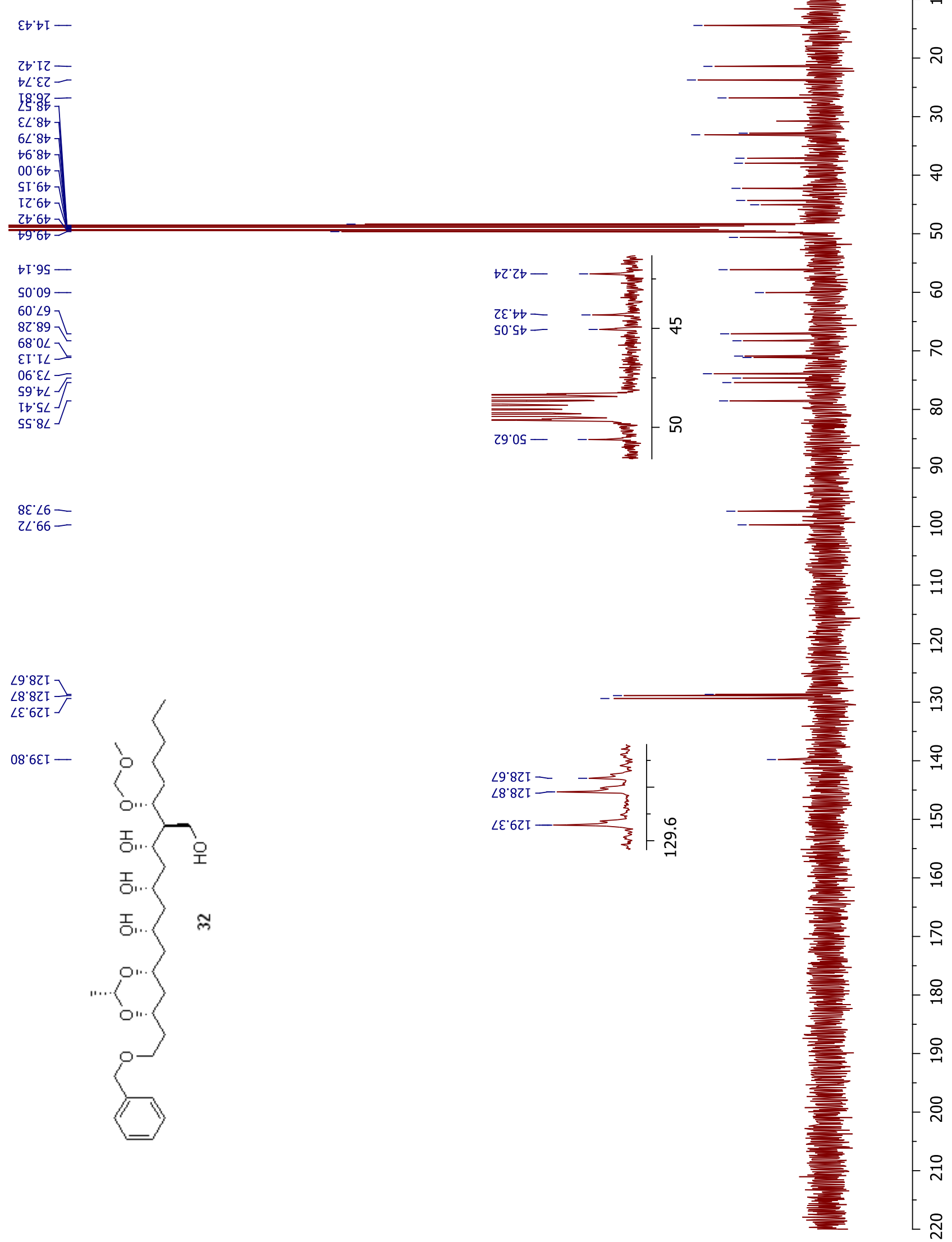




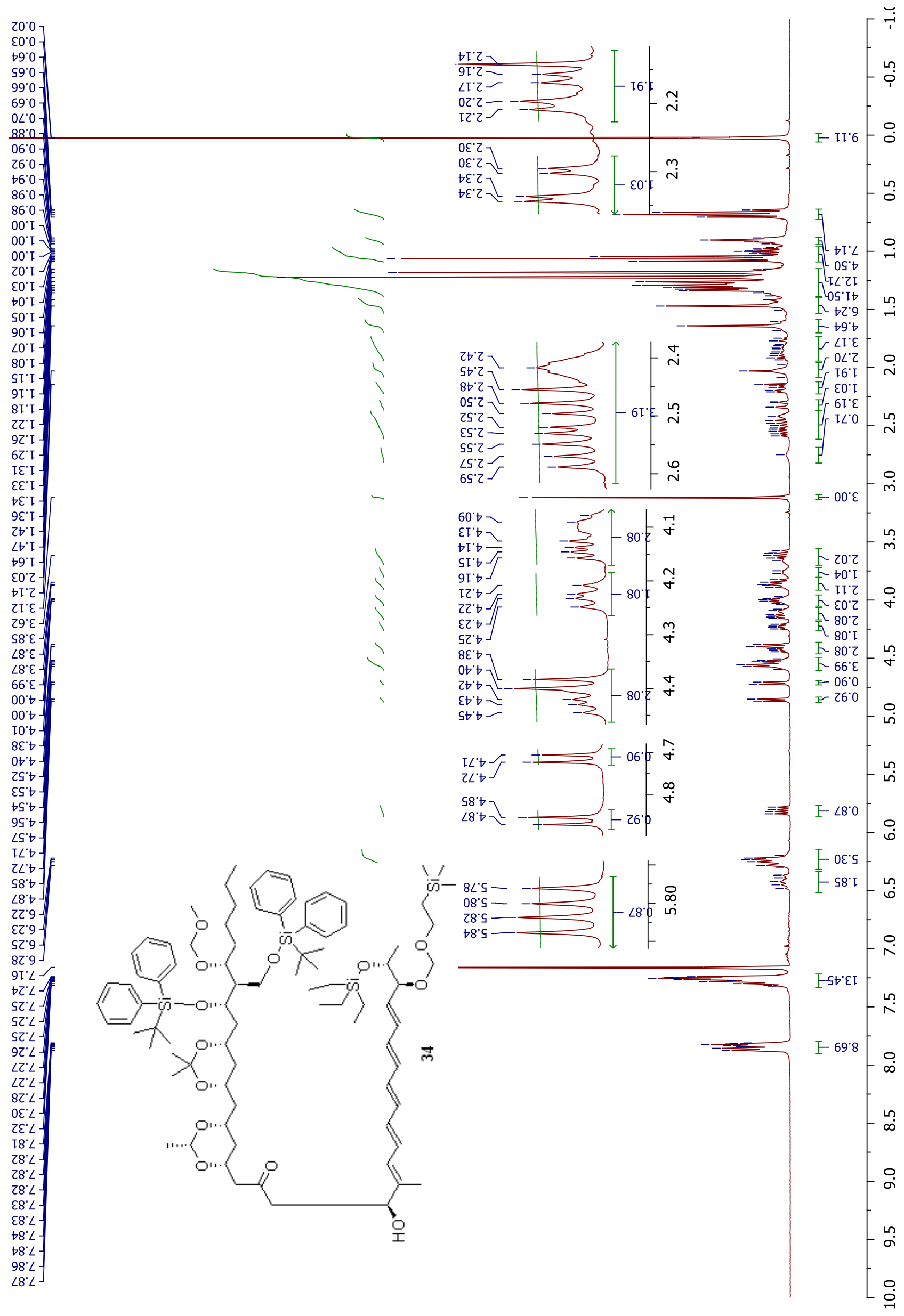



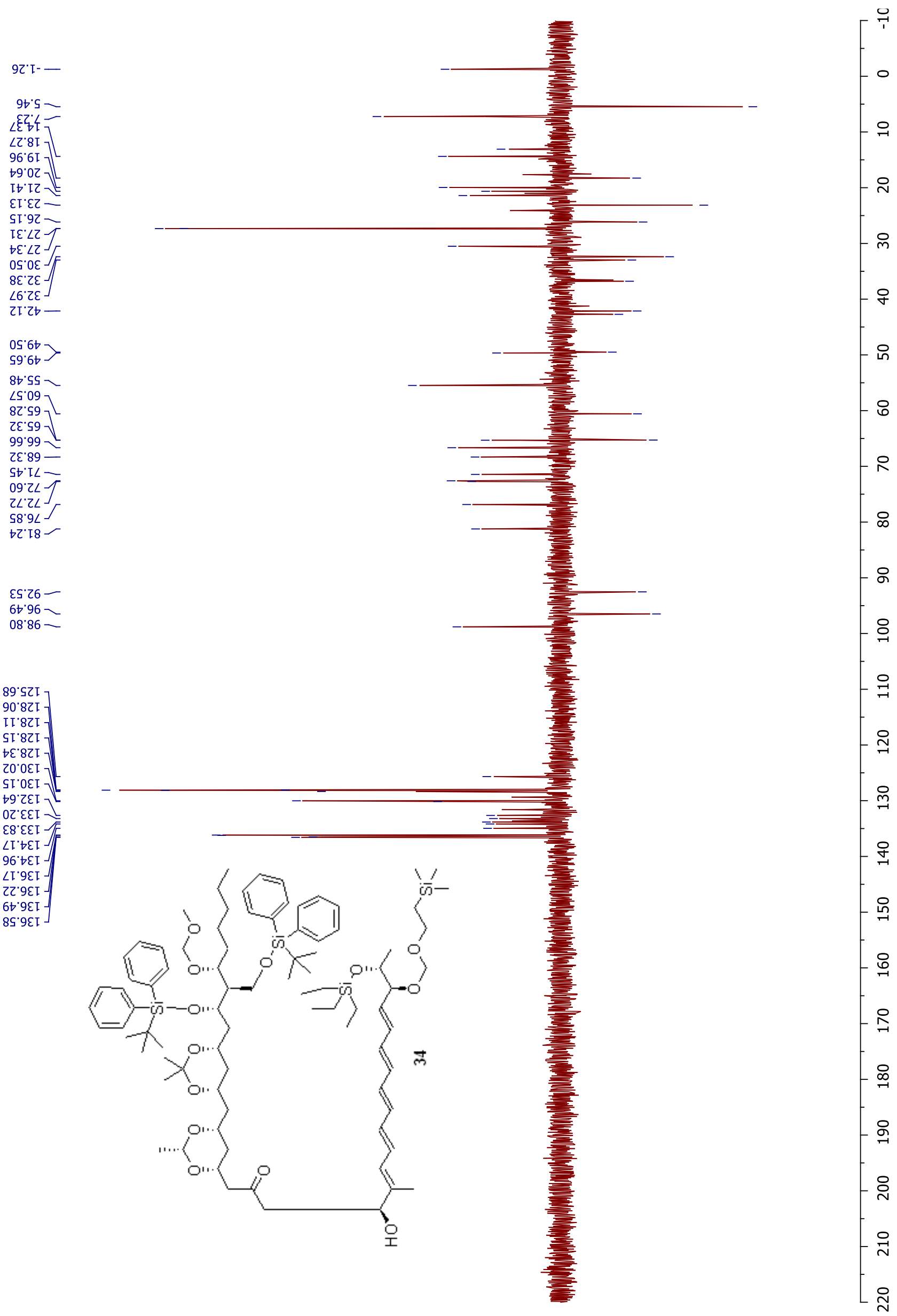


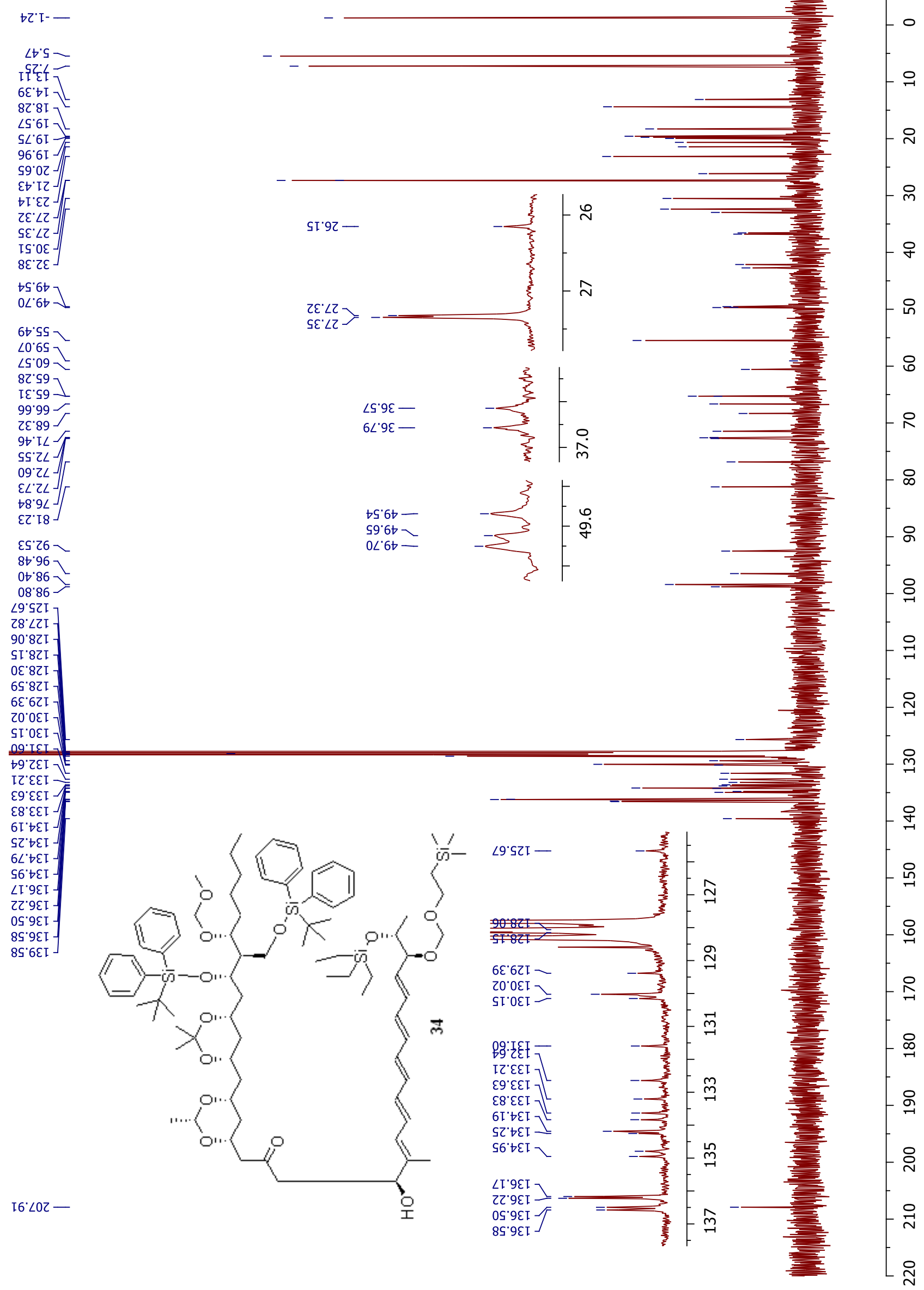




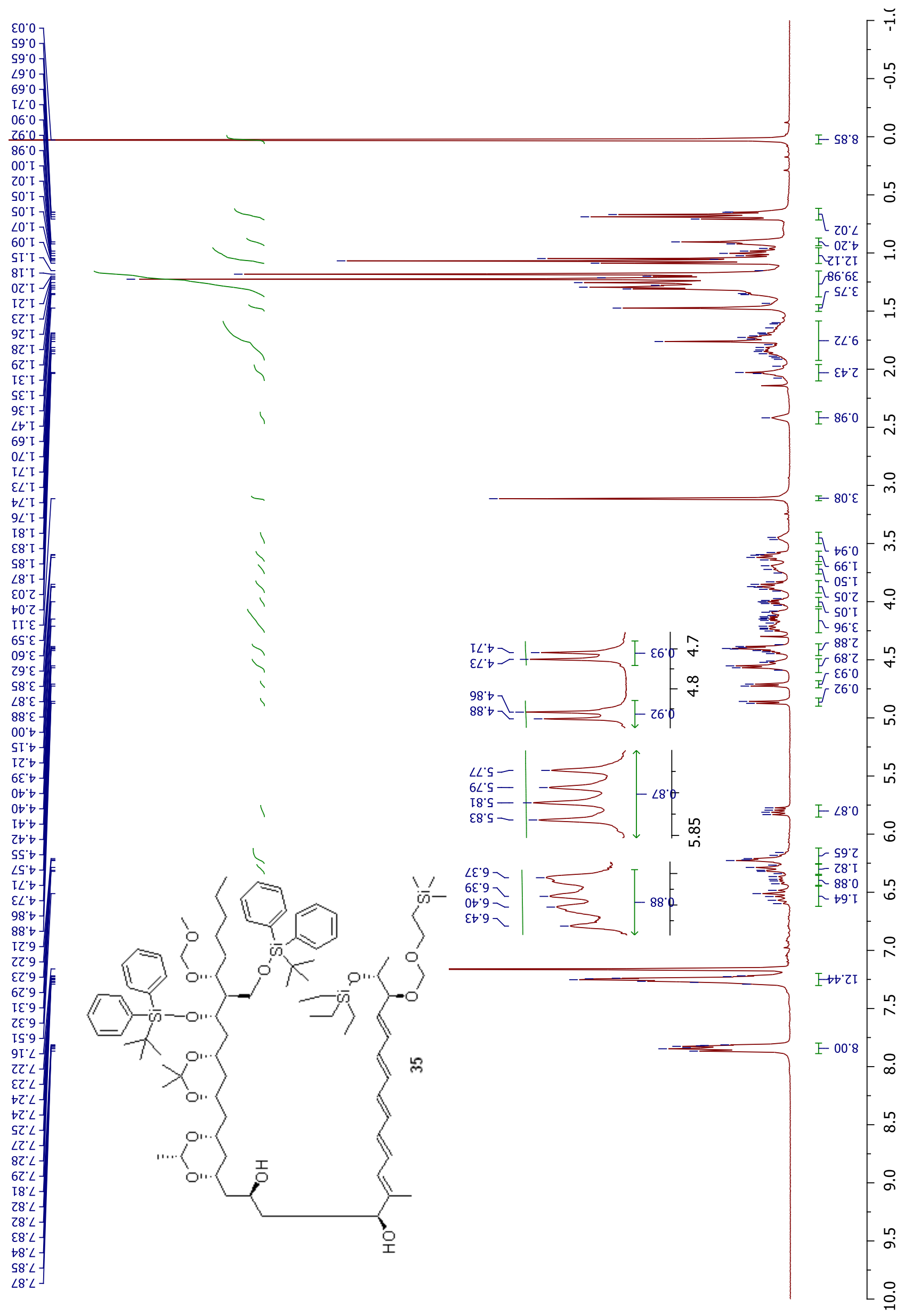



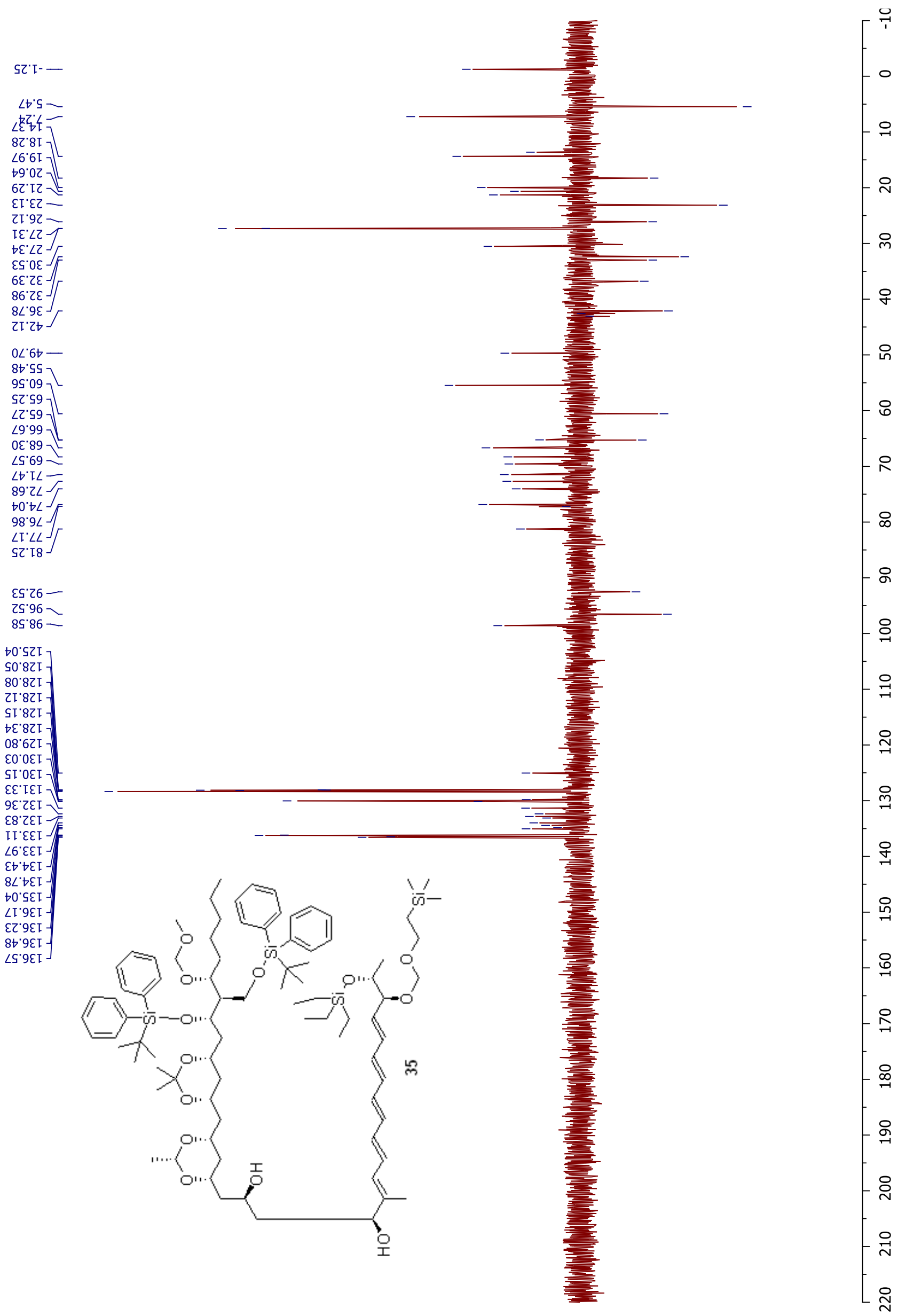


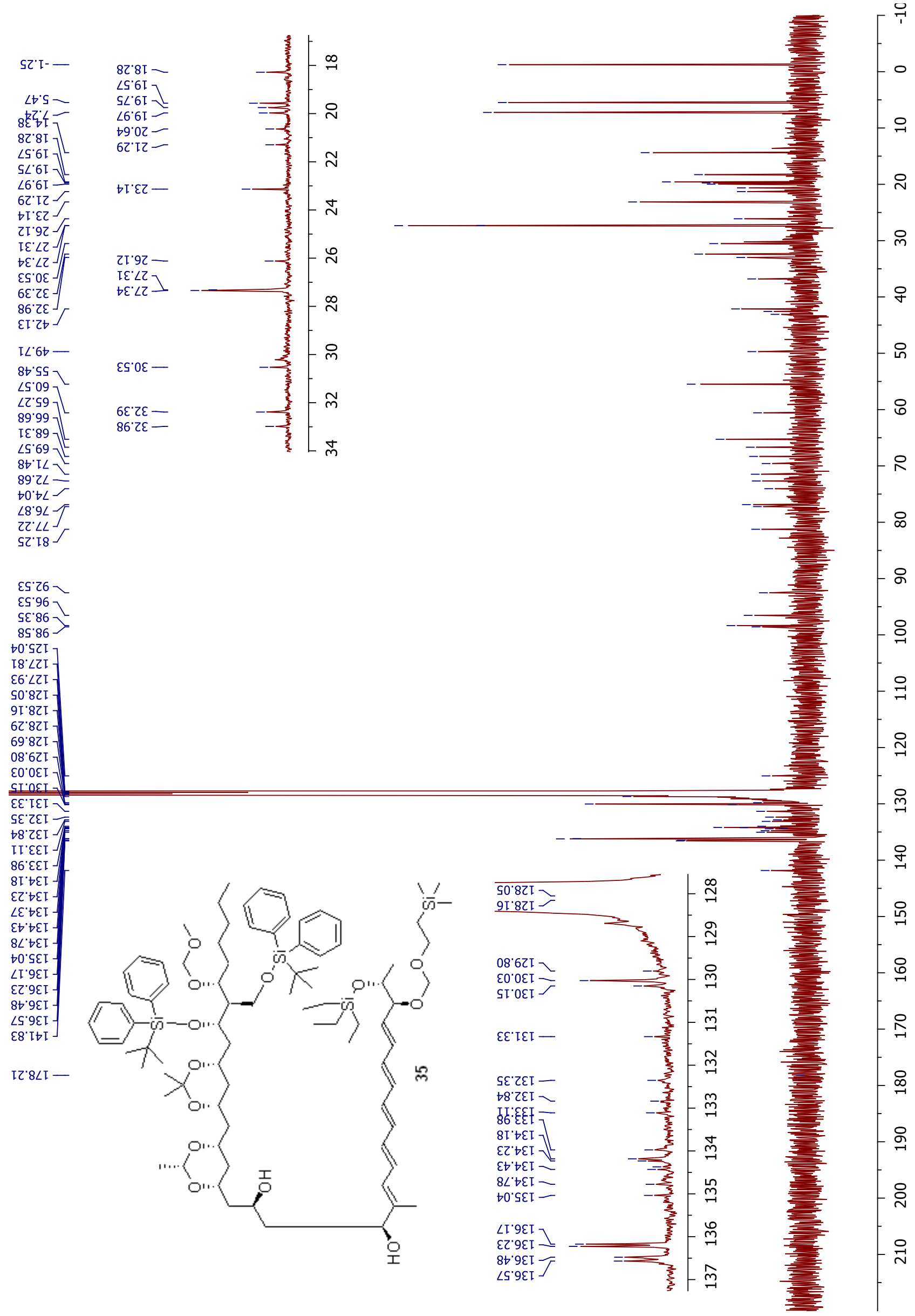




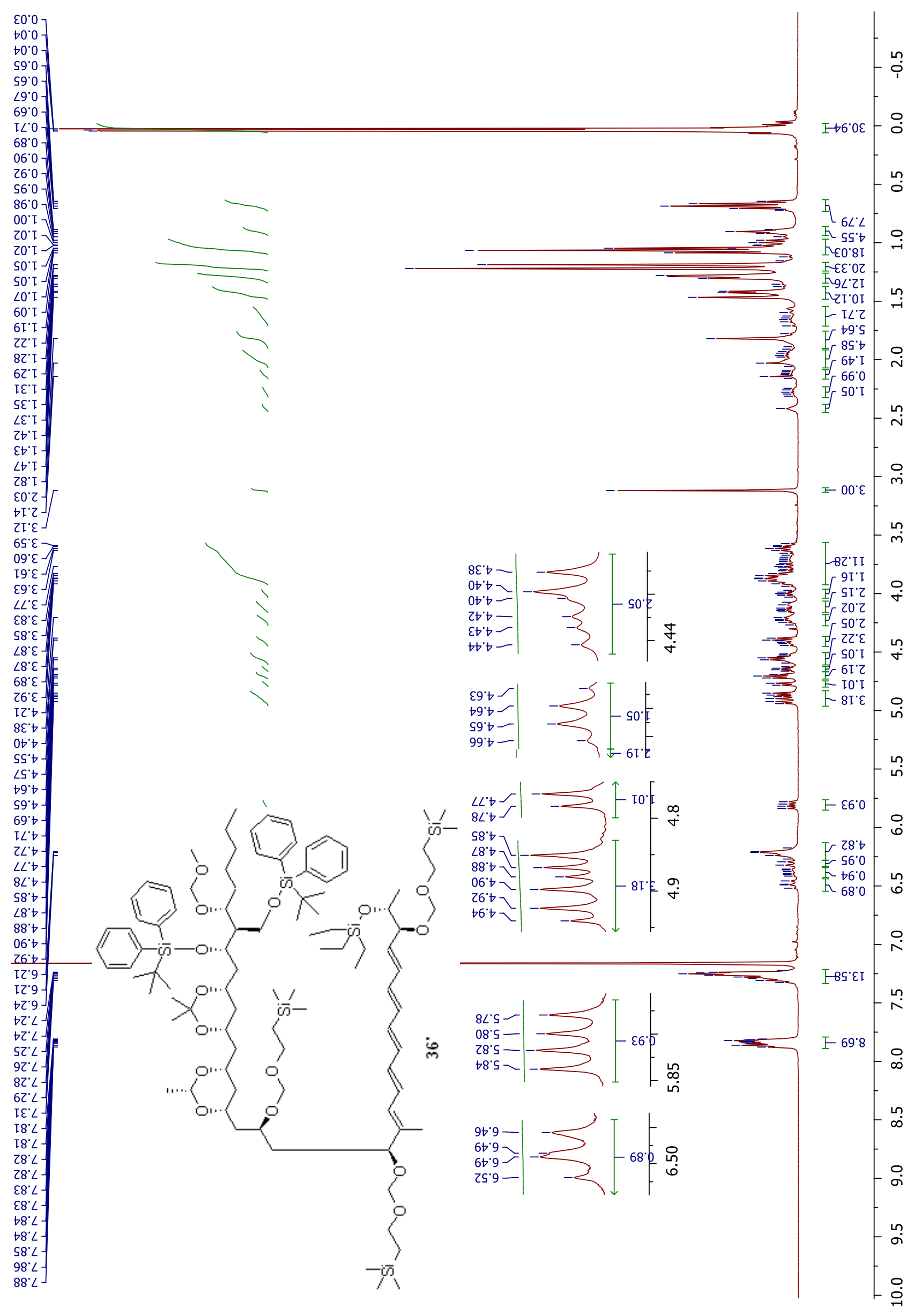


$\begin{array}{ll}t C^{\circ} & - \\ 8 I^{\circ} \cdot- & -\end{array}$

$\angle \mathrm{C}_{\mathrm{D}} \mathrm{I}_{\mathrm{S}}$

SC $\angle 2$

เt'2 I

$6 \varepsilon^{\circ} \circ[$

$82.81]$

$9 \varepsilon^{\circ} 8[]$

โ0.02

t9.02-

IL' IZ

st. 92$]$

ง $\angle Z]$

ऽs $0 \varepsilon-$

$6 \varepsilon " z \varepsilon-$

98:

29:6z-

$\angle S^{\circ} 09$

$\angle Z$ 'S9

ธع' 59$]$

$9<\mathrm{S} 9]$

$99.99-$

حE' 89 -

9t' $\mathrm{T} L-\bar{F}$

$8 L^{\prime} Z L-1$

$\left.\begin{array}{l}0 t^{2} \varepsilon L \\ 58^{\circ} 9 L\end{array}\right]$

IZ' 18 与

25.26

$22.96-$

$6 t^{\circ} 96$ ك

$\angle L ' 86$

t0.82T

II' $82 \mathrm{~T}$

SI $8 Z \mathrm{ZT}$

$8 \mathrm{Z}^{\circ} 8 \mathrm{ZT}$

SE' $8 Z \mathrm{I}$

6S'8ZT

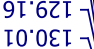

$80^{\circ} 0 \varepsilon \mathrm{E}$

$\rightarrow$ †'OEI

โ 9 ' IEI -

$69^{\circ} \mathrm{LI}$ -

乙Е' $\varepsilon \varepsilon \tau-$

$08 . \varepsilon \varepsilon T$ -

I I $\forall E I-$

$\downarrow 6^{\circ} \rightarrow \varepsilon I-$

$\angle I \cdot 9 \varepsilon \mathrm{I}$

乙乙'9દโ

IS'9Eโ

85.9عI

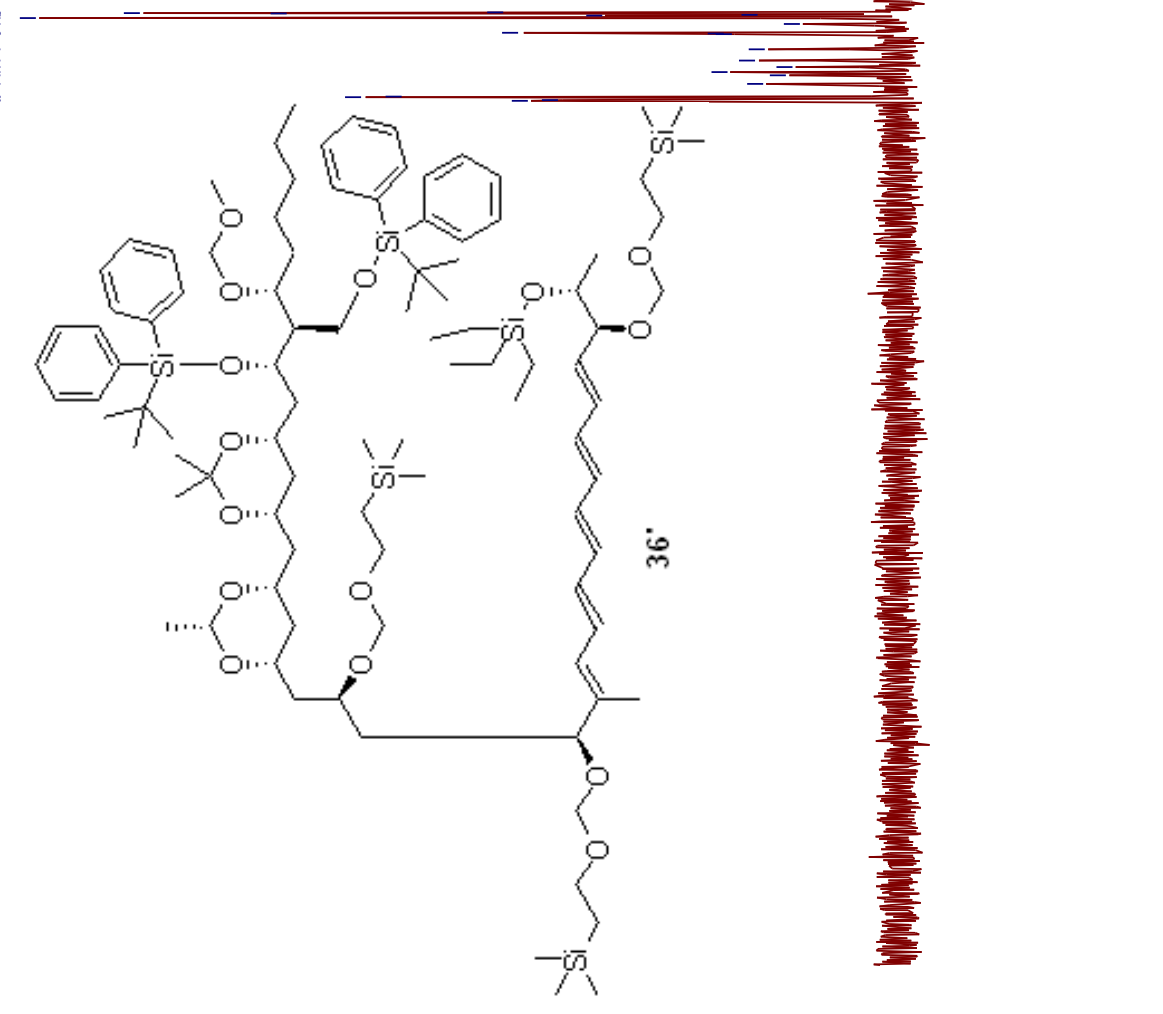




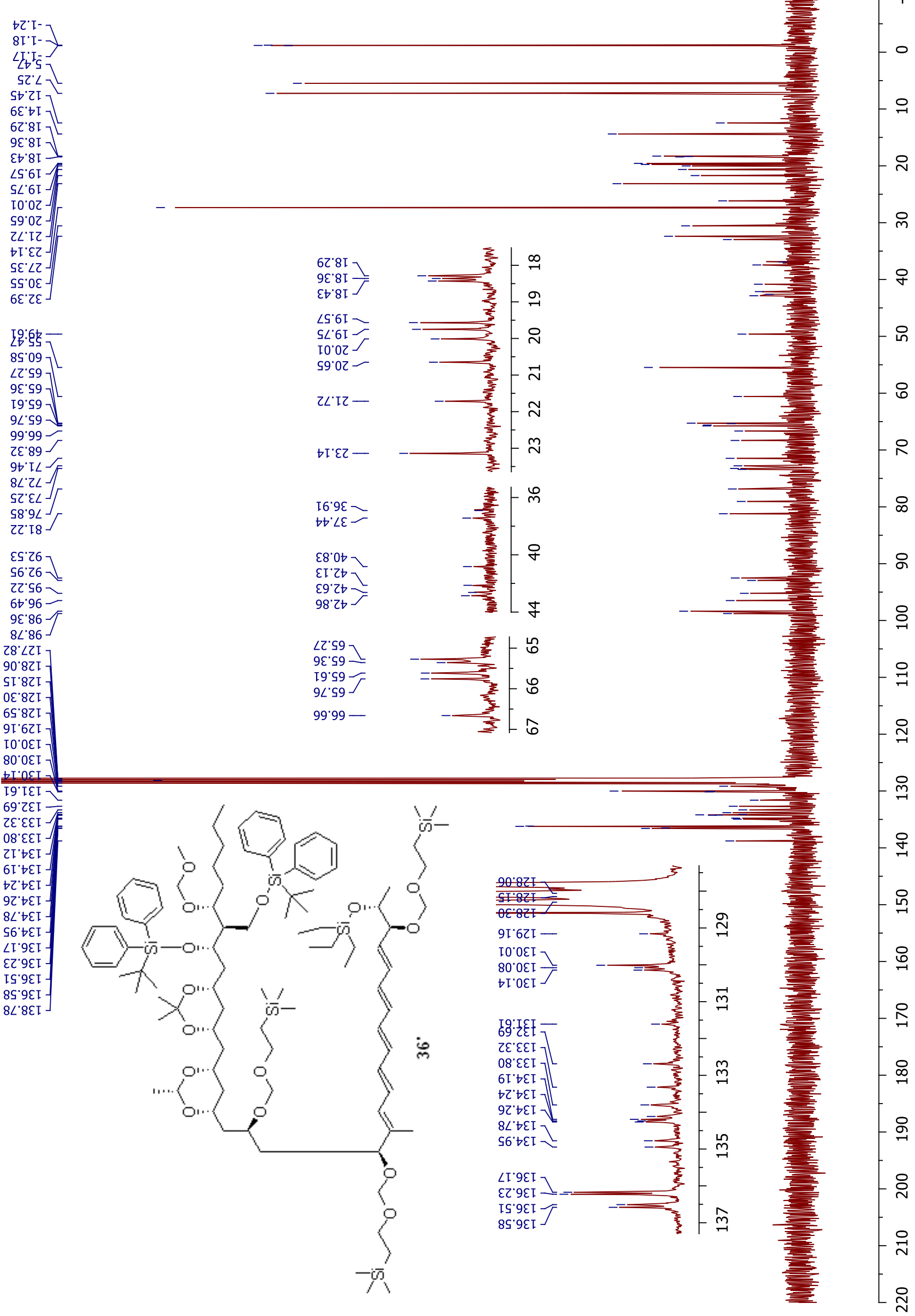




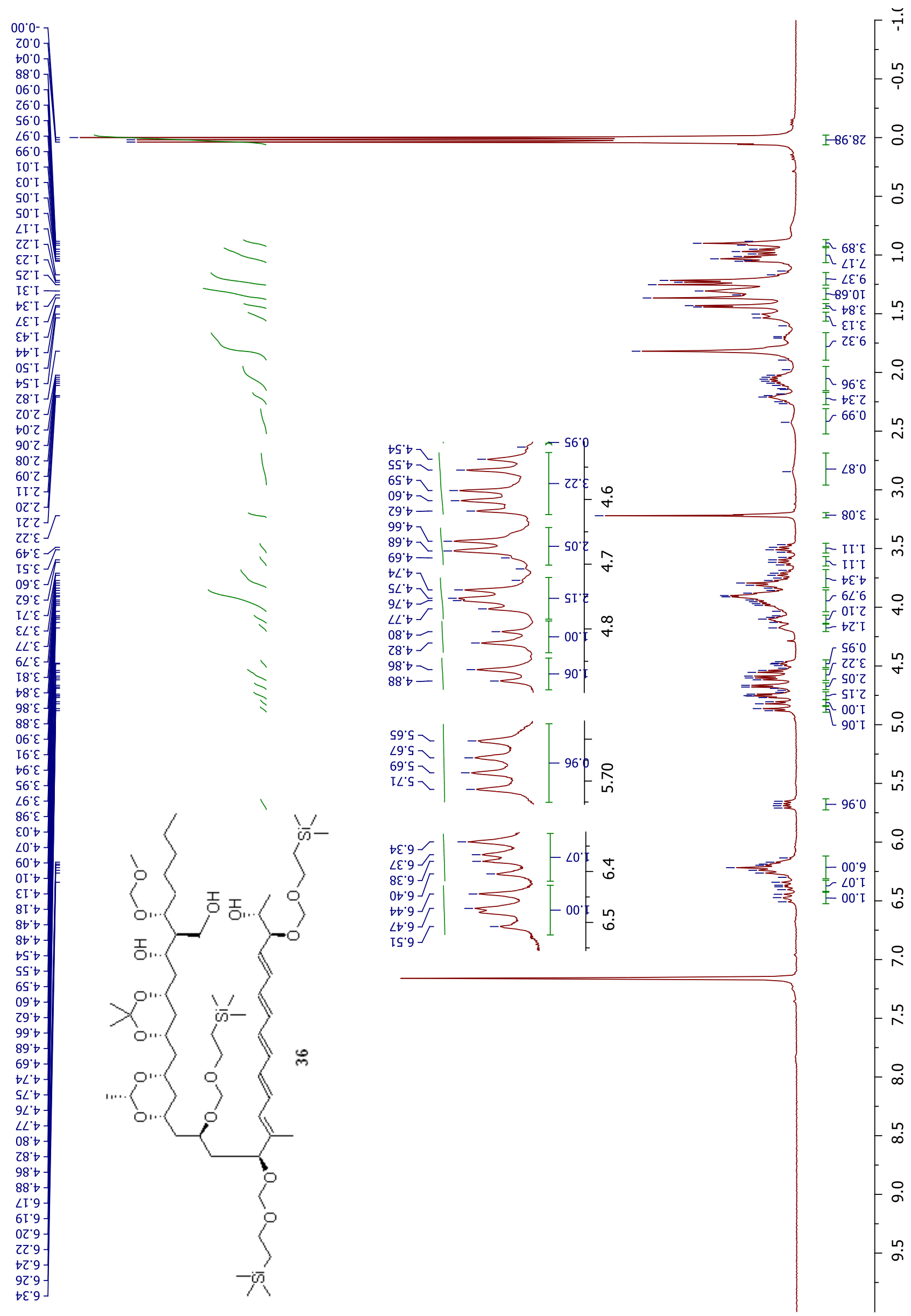




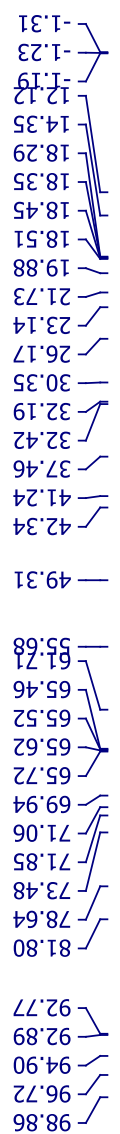
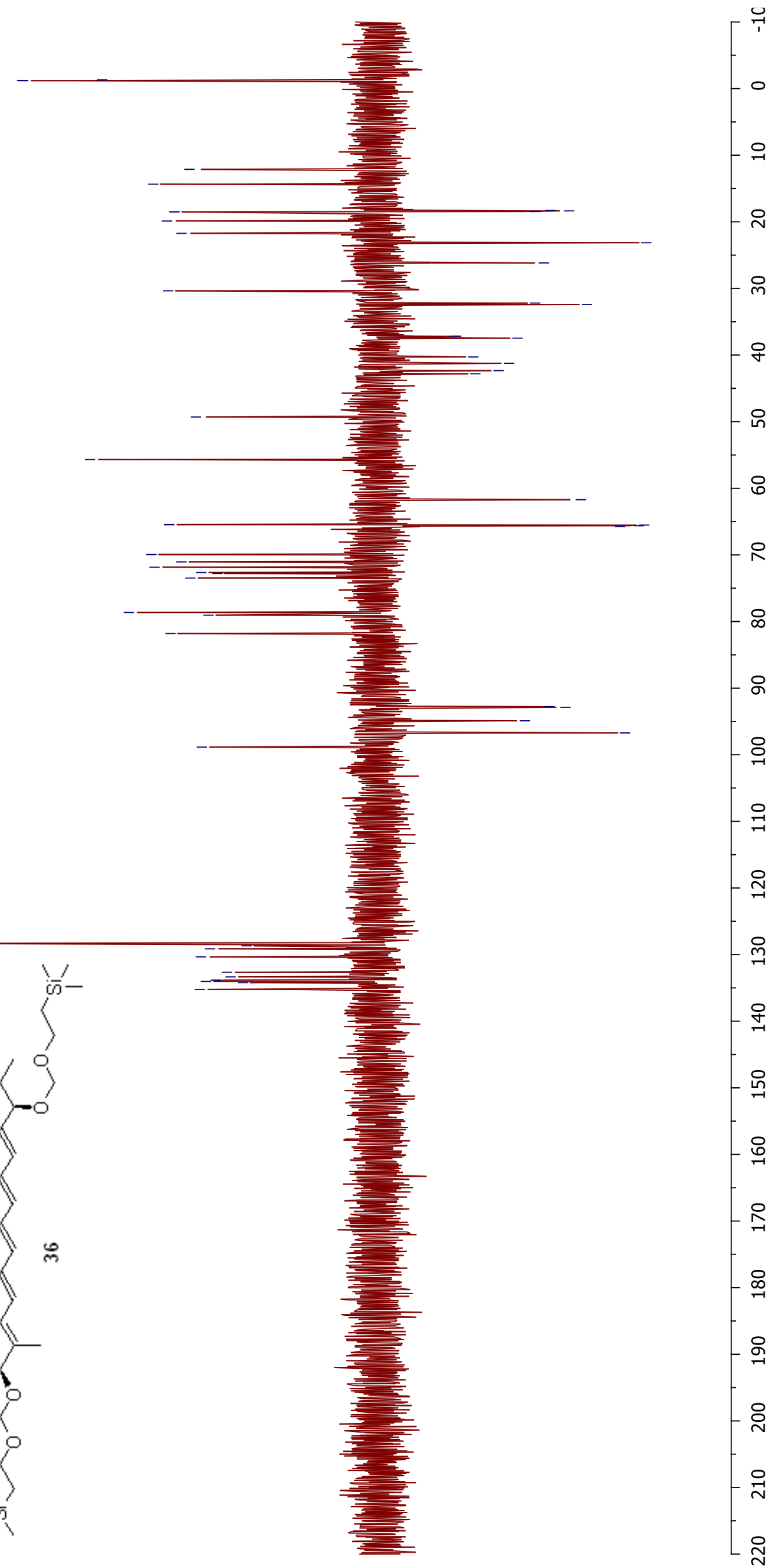

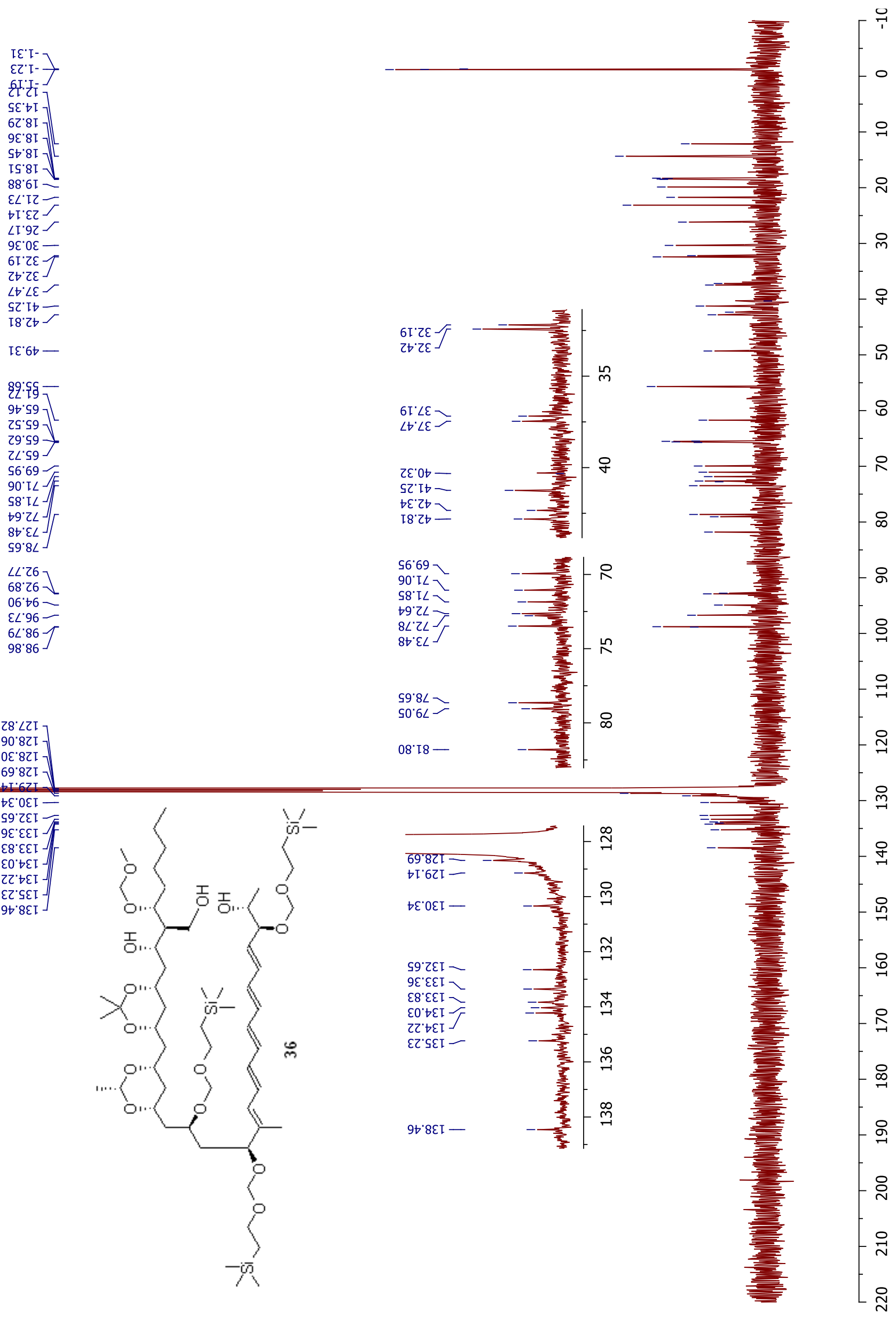